\title{
WEAK SYSTEMS OF GANDY, JENSEN AND DEVLIN
}

\author{
A. R. D. MATHIAS*
}

\section{Table of Contents}

$\S$

Abstract

0 Introduction

\section{Part I}

1 Formulation of the various systems

2 Theorems of various systems 13

On ReS and finite sets 13

Proof that HF models ZF - Infinity 16

On GJ and the class of rudimentary functions $\quad 20$

Companions and the Gandy-Jensen Lemma 21

A single generating function for $\operatorname{rud}(u) \quad 22$

Other remarks on GJ 23

On fReR 25

On ReRI 26

On KP 27

3 Remarks on transitive models 27

4 Models of ReS 30

A model of ReS with full separation omitting $[\omega]^{1}$ and $[\omega]^{2} \quad 31$

5 Models of DB 33

A model of $D B$ omitting $[\omega]^{3} \quad 35$

A failure of $\bigcup$ " $\quad 36$

A failure of Seq $\quad 37$

6 Models of GJ 38

7 Models of fReR and beyond 39

A class model of $Z+$ TCo omitting most ordinals $\quad 39$

* Centre de Recerca Matemàtica, Bellaterra, Catalonia and ERMIT, Université de la Réunion. 


\section{Part II}

8 Adding $\mathcal{S}(x) \in V$ to these systems 41

Normal forms for $\Delta_{0, \mathcal{S}}$ formulae $\quad 42$

Self-strengthening of DS 46

Self-strengthening of GJS 48

Self-strengthening of fReRS 49

Self-strengthening of ReR $\quad 50$

Self-strengthening of KPI $\quad 52$

9 The Gandy sequence $\quad 53$

The model $J_{2}$

A model of $Z$ failing rank-bounded replacement $\quad 57$

10 Mending the flaws in Devlin's book 58

Errors in Chapter I $\quad 59$

Errors in Chapters II 64

Errors in Chapters VI 66

The cure in $D S \quad 67$

The cure in GJI 67

Conclusion 68

\section{Part III}

11 Gandy's inexact remarks 68

12 A model of $\mathrm{Z}+$ full foundation in which TCo fails $\quad 70$

13 AxPair and AxSing $\quad 72$

14 A question of MacAloon on rudimentary closures $\quad 74$

15 An application to Gandy numerals $\quad 77$

$\begin{array}{ll}\text { Acknowledgments and References } & 79\end{array}$ 
ABSTRACT. In Part I, we formulate and examine some systems that have arisen in the study of the constructible hierarchy; we find numerous transitive models for them, among which are supertransitive models containing all ordinals that show that Devlin's system BS lies strictly between Gandy's systems PZ and BST'; and we use our models to show that BS fails to handle even the simplest rudimentary functions, and is thus inadequate for the use intended for it in Devlin's treatise. In Part II we propose and study an enhancement of the underlying logic of these systems, build further models to show where the previous hierarchy of systems is preserved by our enhancement; and consider three systems that might serve for Devlin's purposes: one the enhancement of a version of BS, one a formulation of Gandy-Jensen set theory, and the third a subsystem common to those two. In Part III we give new proofs of results of Boffa by constructing three models in which, respectively, TCo, AxPair and AxSing fail; we give some sufficient conditions for a set not to belong to the rudimentary closure of another set, and thus answer a question of McAloon; and we comment on Gandy's numerals and correct and sharpen other of his observations.

\section{INTRODUCTION}

During the 1960's, as knowledge of the constructible hierarchy advanced, pre-eminently through the work of Jensen [J1] [J2], there was a drive to study various weak systems of set theory, all weaker than that of Kripke-Platek. Those systems included $\Delta_{0}$ separation but weakened $\Delta_{0}$ collection in various ways, and their purpose was to give a finer account of the growth of the constructible hierarchy. As is well-known, this move has been extraordinarily fruitful.

Gandy $[\mathrm{G}]$ proposed four systems which he called PZ (for "predicative Zermelo"), BST', BRT and PZF. and which he proved to be strictly ascending in strength. Devlin in his treatise [D] proposed a further system, which he called BS.

We shall, starting in $§ 1$, introduce new names for those five systems and others which have suggested themselves, but shall use the old in this introduction.

So, roughly, PZ is a weak base theory plus $\Delta_{0}$ separation. BS adds cartesian product to that. BST' is the result of adding an axiom of infinity to Gandy's theory BST, of which the transitive models are precisely the rudimentarily closed sets. BRT has what Gandy calls the bounded replacement axiom; and PZF has $\Delta_{0}$ replacement, making it weaker than but close to and equiconsistent with the system of Kripke-Platek with an axiom of infinity. We shall also look briefly at what Gandy would have called the bounded collection axiom, and at our preferred formulation of the system of Kripke and Platek. 
When, in the next section and later, we give precise formulations of systems, we shall put names of systems and axioms in nine-point sans-serif type to indicate that it is our particular formulations that are being discussed, as defined either in this paper or in [M2]. In our formulations we shall change some of Gandy's terminology and notation, since Gandy uses the term "basic" for the functions that Jensen called "rudimentary"; and further Gandy studies two versions of the axiom of replacement, calling the one "basic" and and the other "bounded", an unfortunate combination of adjectives as both begin with 'b'. Therefore we shall follow Jensen's usage, often shortening "rudimentary" to "rud", and shall use "RR" to name what Gandy called the basic replacement axiom. We shall reserve the word "basic" for a proper subclass $\mathcal{B}$ of the class $\mathcal{R}$ of rudimentary functions, namely those generated by composition from Gödel's functions $\mathcal{F}_{1}, \ldots, \mathcal{F}_{8}$, and we shall use "flat" where Gandy used "bounded" in naming axioms.

In discussing these systems it will, as in The Strength of Mac Lane Set Theory [M2], at times be necessary to maintain a careful distinction between three levels of language, which we call the metalanguage, which is English, the language of discourse, which is a language of set theory formulated with atomic predicates $\in$ and $=$, and various object languages, again set-theoretical in nature, with atomic predicates symbolised by $\epsilon$ and $=$. We use Fraktur lower case letters $\mathfrak{k}, \mathfrak{l}, \mathfrak{m}, \ldots$ for concrete integers, which are quantified only in the metalanguage, and the corresponding terms for them in the language of discourse. This visual aid may be used to mark the distinction between a system $\mathrm{T}$ being able, for each $\mathfrak{k}$, to prove some statement $\Phi(\mathfrak{k})$ and being able to prove $\forall k \Phi(k)$.

\section{Three areas of uncertainty in the choice of axioms}

The above authors differ in their treatment of the scheme of foundation: Gandy makes no mention of foundation in his formulations, whereas Devlin calls for the full scheme of foundation in his. Without foundation, his system is intermediate between PZ and BST'. The question of the amount of foundation possessed by a system is not idle: in our paper [M2] we showed that in terms of consistency strength $\Pi_{2}$ foundation is in some cases strictly stronger than $\Pi_{1}$ foundation - see Metacorollary 9.21 and Metatheorem 9.34 of [M2] - and there is evidence that $\Pi_{1}$ foundation is the "right" amount to have in formulating the system of Kripke-Platek; see Corollary 1.22(ii) and Proposition 3.14 (ii') of [M2]. The investigations of the present paper suggest that $\Pi_{1}$ foundation is also the "right" amount to have in these weaker systems.

A second area of uncertainty is the axiom of transitive containment, TCo, which asserts that every set is a member of a transitive set. It was 
shown by Boffa [B1],[B2] that TCo is not provable in Zermelo set theory: we give a new proof of that result in Section 12. TCo is, however, provable in our formulation of Kripke-Platek.

Finally it is of interest to see to what extent the axiom of infinity can be avoided.

So our policy will be, at least initially, to exclude the "special" axioms of infinity and transitive containment from the general axioms of our systems, and explicitly to note each use of those special axioms as it occurs. As for foundation, we shall include the scheme of $\Pi_{1}$ foundation in our systems, and draw attention to areas where foundation can be avoided, and where the full scheme of foundation is required.

In many sections of the paper, our focus will be chiefly not on the consistency strength of the various theories but on constructing transitive models for them; and in such models, the full scheme of foundation will be inherited from our ambient set theory. Further TCo and AxInf will be true in most of our models. We remark that we are not in this paper concerned to find the minimal ambient set theory in which our examples can be built. ZF is certainly too strong; $Z+K P$ is usually enough, apart from the occasional appeal to the existence of $V_{\omega+\omega}$ and similar sets. The axiom of choice is used only in a very few peripheral remarks.

\section{Some differences}

Some of the differences between these systems may be illustrated with the help of certain sets functioning as litmus paper:

0.0 Definition We write $\mathcal{S}(x)$ for the set of finite subsets of $x$; for each $\mathfrak{k}>0$, $[\omega]^{\mathfrak{k}}$ for the class of subsets of $\omega$ of size $\mathfrak{k} ; \mathbf{H F}$ for the class of hereditarily finite sets, which in appropriate set theories will coincide with the classes notated $V_{\omega}, L_{\omega}$ and $J_{1}$; EVEN for the class of even numbers, ACK for the Ackermann relation on $\omega$, defined as $\left\{(m, n)_{2} \mid 2^{m}\right.$ is one of the summands in the expression of $n$ as a sum of powers of 2$\}$; and $\mathcal{G}_{+}$for the graph of integer addition, defined as the class $\left\{(p, m, n)_{3} \mid m+n=p\right\}$.

We shall see that PZ cannot prove the existence even of $[\omega]^{1}$; BS can prove the existence of $[\omega]^{1}$ and $[\omega]^{2}$ but not of $[\omega]^{3}$; BST' can prove the existence of each $[\omega]^{\mathfrak{k}}$; BST' with $\Pi_{1}$ foundation can prove that $\left.\forall k \omega\right]^{k} \in V$ but cannot prove the existence of $\mathcal{S}(\omega)$; BRT can prove the existence of $\mathcal{S}(\omega)$ but not of $\mathbf{H F}$; and PZF proves the existence of HF. Further, we shall see that BRT proves that $\mathcal{G}_{+}$is a set but that BST' fails to do so.

\section{The contents of the paper}

In the first of the three parts of the paper, we shall formulate, in $\S 1$ eight systems, with variants, and note in $\S 2$ various results provable in them. 
In $\S 3$, we review some simple techniques for building transitive models of weak systems. In the next four sections, we work through the systems in order of increasing strength, summarising Gandy's model-theoretic constructions and giving new ones of our own; our models will demonstrate the unprovability of various results.

The second part begins with the heavily syntactic $\$ 8$, in which we examine the result of strengthening our previous systems by uniformly adding an axiom of infinity and the principle that the class of all finite subsets of any given set is a set; and study the effect of enhancing those strengthened systems by adding limited quantifiers of the form "for some finite subset of $a$ " and "for all finite subsets of $a$ ". In $\S 9$, we give further models illustrating the limitations of our strengthened systems. Then in $\S 10$ we turn to an examination of Devlin's book Constructibility, of which certain passages have been known since its publication to be problematical; we use our models to shed light on those passages, and draw attention to three of our systems that might serve Devlin's purposes better than the system BS.

We begin the final part of the paper by showing in $\$ 11$ that Gandy's remarks concerning certain variants of his systems are not correct. In $\S 12$, we return to model-building and give a new proof of the result of Boffa that TCo is not provable in Zermelo set theory; $§ 13$ looks briefly at the axiom of pairing; in $\S 14$ we find an answer to a question raised by McAloon in the 1970 's by giving criteria for one set not to lie in the rudimentary closure of another; finally in $\S 15$ we apply the technique of $\S 14$ to show that the set of Gandy numerals is not in the rudimentary closure of $\omega$.

\section{FORMULATIONS OF THE VARIOUS SYSTEMS}

We start with enough syntax to introduce the axioms of our first, very weak system, and to define for each $\mathfrak{n}$ the ordered $\mathfrak{n}$-tuple; then we shall enlarge the syntax to include some convenient extensions of the class-forming operator, and shall then be able to enunciate in the language of discourse the axioms of the systems we intend to study.

We begin therefore with two undefined binary relations $\epsilon,=$; propositional connectives $\neg, \mathrm{V}, \&, \Longrightarrow, \Longleftrightarrow$; unrestricted quantifiers $\forall x, \exists x$; restricted quantifiers $\forall x: \in y, \exists x: \in y$, where $x$ and $y$ are not permitted to be the same letter; and the class-forming operator $\{\cdot \mid \ldots\}$. The rules of formation are the usual ones of classical logic.

We then define a $\Delta_{0}$ formula or a $\Delta_{0}$ class to be one containing no unrestricted quantifiers; a $\Pi_{1}$ formula is one of the form $\forall x \mathfrak{A}$ where $\mathfrak{A}$ is $\Delta_{0}$; a $\Sigma_{2}$ formula is one of the form $\exists y \mathfrak{B}$ where $\mathfrak{B}$ is $\Pi_{1}$; a $\Sigma_{1}$ formula is one of the form $\exists x \mathfrak{A}$ where $\mathfrak{A}$ is $\Delta_{0}$, and so on. 
We have the usual axioms of classical propositional and predicate logic; we define the result of substituting one variable for another, indicated informally by such usages as $\mathfrak{A}(x)$ and $\mathfrak{A}(y)$; or of substituting a class for a variable; and we have the Church conversion schema:

$$
x \in\{y \mid \mathfrak{A}(y)\} \Longleftrightarrow \mathfrak{A}(x) .
$$

With this syntax, we may give axioms for our first, very weak, system:

$\mathrm{S}_{0} \quad$ The axiom of extensionality, $[\forall x: \in a x \in b \& \forall x: \in b x \in a] \Longrightarrow a=b$, and axioms of empty set, pair set, difference and sumset (or union):

$$
\varnothing \in V, \quad\{x, y\} \in V, \quad x \backslash y \in V, \quad \bigcup x \in V .
$$

In this system we introduce, successively, ordered $\mathfrak{k}$-tuples, in the manner usually credited to Wiener and Kuratowski:

$$
\begin{aligned}
\left(y_{1}\right)_{1} & =_{\mathrm{df}} y_{1} \\
\left(y_{1}, y_{2}\right)_{2} & =_{\mathrm{df}}\left\{\left\{y_{1}\right\},\left\{y_{1}, y_{2}\right\}\right\} \\
\left(y_{1}, y_{2}, y_{3}\right)_{3} & { }_{\mathrm{df}}\left(y_{1},\left(y_{2}, y_{3}\right)_{2}\right)_{2} \\
\left(y_{1}, y_{2}, y_{3}, y_{4}\right)_{4} & { }_{\mathrm{df}}\left(y_{1},\left(y_{2}, y_{3}, y_{4}\right)_{3}\right)_{2} \\
\left(y_{1}, y_{2}, y_{3}, y_{4}, y_{5}\right)_{5} & ={ }_{\mathrm{df}}\left(y_{1},\left(y_{2}, y_{3}, y_{4}, y_{5}\right)_{4}\right)_{2}
\end{aligned}
$$

1.0 REMARK Thus all WK-tuples are generated from the single binary function $\{x, y\}$.

We may now develop the usual theory of relations, $\mathfrak{k}$-ary functions and so on: we treat functions as a subclass of their image $\times$ their domain. We shall see that these weak systems are sensitive to the choice of implementation of function, and so it is necessary to distinguish notationally between concepts that "the working mathematician" would often conflate. Thus we adopt a policy of writing ${ }^{3} X$ for the set of 3 -sequences of members of $X$, reserving $X^{3}$ for the set of WK 3-tuples of members of $X$; thus $\omega^{3}=\omega \times(\omega \times \omega)$.

1.1 It is convenient formally to enlarge the syntax to permit certain classes with quantified terms, namely those where the terms are WK-tuples: where there might otherwise be ambiguity, we indicate the variables to be quantified in a list placed subscript to the vertical bar, for example:

$$
\left\{\left.(x, y)_{2}\right|_{x, y} \mathfrak{A}(x, y)\right\} ; \quad\left\{\left.(x, a)_{2}\right|_{x} \mathfrak{A}(x, a)\right\}
$$


The first of those will equal $\left\{z \mid \exists x \exists y\left[z=(x, y)_{2} \& \mathfrak{A}(x, y)\right]\right\}$; the second, $\left\{z \mid \exists x\left[z=(x, a)_{2} \& \mathfrak{A}(x, a)\right]\right\}$ for the given $a$ : such equalities are accomplished by adding the following scheme to our system:

$$
x \in\left\{\left.\left(y_{1}, \ldots, y_{\mathfrak{k}}\right)_{\mathfrak{k}}\right|_{y_{1}, \ldots, y_{\mathfrak{k}}} \mathfrak{A}\right\} \Longleftrightarrow \exists y_{1} \ldots \exists y_{\mathfrak{k}}\left[x=\left(y_{1}, \ldots, y_{\mathfrak{k}}\right)_{\mathfrak{k}} \& \mathfrak{A}\right] .
$$

1.2 We informally permit classes with other quantified terms, for example $\left\{\left.\bigcup x\right|_{x} x \in a\right\}$.

1.3 Definition Foundation, the axiom of (set) foundation, is $x \neq \varnothing \Longrightarrow$ $\exists y: \in x x \cap y=\varnothing$.

$\mathrm{S}_{0}^{\prime} \quad \mathrm{S}_{0}+$ Foundation

\section{A calculus of $\Delta_{0}$ terms}

1.4 Definition We call a term $A$, possibly with free variables, T-semisuitable, where $\mathrm{T}$ is some system of set theory, if whenever $\Phi$ is $\Delta_{0}$, and the variable $w$ is not free in $A$, then $\forall w: \in A \Phi$ is $\Delta_{0}^{\top}$, that is, equivalent over $\mathrm{T}$ to a $\Delta_{0}$ formula. If in addition, $\mathrm{T}$ proves that $A$ is a set, we call $A$ $\mathrm{T}$-suitable.

1.5 REMARK $\mathrm{S}_{0}$ is adequate for the development of a surprisingly large number of suitable terms. In particular, $\bigcup x$ is $\mathrm{S}_{0}$-suitable, as is each $\bigcup^{\mathfrak{l}} x$. $\mathrm{S}_{0}$ easily proves that if $x=(y, z)_{2}$, then $y \in \bigcup^{2} x$ and $z \in \bigcup^{2} x$; hence if $\mathfrak{A}$ is $\Delta_{0}$ then the class $\left\{\left.\left(y_{1}, y_{2}, \ldots, y_{\mathfrak{k}}\right)_{\mathfrak{k}}\right|_{y_{1}, y_{2}, \ldots, y_{\mathfrak{k}}} \mathfrak{A}\right\}$ is equal, provably in $\mathrm{S}_{0}$, to a $\Delta_{0}$ class.

With Foundation added, the formulation of "ordinal" becomes $\Delta_{0}$ and much of the elementary theory of ordinals can then be developed.

1.6 REMARK Gandy in $[\mathrm{G}]$ proves that the term $\omega$ is $\mathrm{S}_{0}^{\prime}$-semi-suitable in that if $\Phi$ is $\Delta_{0}$ then the formula $\exists y: \in \omega \Phi$ is equivalent in $\mathrm{S}_{0}^{\prime}$ to a $\Delta_{0}$ formula. His proof will work for appropriate terms for each ordinal strictly less than $\omega^{\omega}$, an interesting ordinal shown by Delhommé [Del] to be the first non-automatic ordinal, but, by [DoMT, page 44, Theorem 38], no further.

1.7 REMARK Gandy [G] and Dodd [Do] have a concept of "substitutable" which is similar to our "suitable" but formulated semantically rather than syntactically. Jensen [J2] and Devlin [Dev] have the same concept but call it "simple". In the present author's opinion, that concept has the danger of blurring the levels of language. If one considers a rudimentary function to be defined by a class of the language of discourse, then implicitly there is a quantification taking place in the meta-language whenever one uses such phrases as "rud closed" or "the class of rud functions". That is scarcely satisfactory, though the situation is saved by defining a rud closed set to be 
one closed under, say, the explicit list of nine functions given in 2.62. What would be better would be to resort to some mild recursion theory, and to list terms of an object language defining certain (set-theoretical) computations, and then when one speaks of closure the quantification is indeed going on in the language of discourse.

Thus it would seem that the axiom TCo, not adopted by Mac Lane, expresses a characteristic of set theory, namely that it is often concerned with computations going on in small portions of the universe, the transitive sets; or the transitive sets closed under pairing functions. Not adopting TCo is a sop to the structuralists; but adopting it is what set theorists should do if they are to be true to their underlying intuitions. The point is linked to the meaning of $\Delta_{0}$ and will recur in Remark 10.1, on page 34 .

\section{Names of systems}

Our policy will be this: if we have a system $\mathrm{X}, \mathrm{X}_{0}$ will mean the variant of that system with no axiom of foundation, no TCo, and no axiom of infinity. Without that subscript, $\Pi_{1}$ foundation will be customary. We use "restricted" to mean $\Delta_{0}$. We use "flat", where Gandy used "bounded", to mean that a certain quantifier limits its variable to subsets of a named set.

Four of our names will reflect the fact that a significant part of the system is the scheme of restricted separation, flat restricted replacement, flat restricted collection or restricted replacement: ReS, fReR, fReC, ReR.

We shall add the letter $I$ to indicate the adjunction of an axiom of infinity, usually in the form $\omega \in V$. In $\S 8$ we shall add the letter $\mathrm{S}$ to existing names to indicate the adjunction of the axiom of infinity and the axiom $\mathcal{S}(x) \in V$. TCo will be listed by name when needed.

\section{Gandy's first system}

Gandy called his weakest system PZ, for "predicative Zermelo", and his strongest PZF, for "predicative Zermelo-Fraenkel". They are both something of a misnomer as he overlooked the power-set axiom; and without that axiom, as shown by Zarach [Z], the difference between replacement and separation-with-collection becomes significant. We use ReS, for "restricted separation".

$\operatorname{ReS}_{0} \quad \mathrm{~S}_{0}$ plus the $\Delta_{0}$ separation axiom: $x \cap A \in V$ for $A$ a $\Delta_{0}$ class.

ReS $\operatorname{ReS}_{0}$ plus the scheme of $\Pi_{1}$ foundation: $A \neq \varnothing \Longrightarrow \exists x: \in A x \cap A=\varnothing$ for $A$ a $\Pi_{1}$ class. 


\section{Devlin's system and variant}

The next system, which we call DB for "Devlin Basic", adds the existence of cartesian product to $\mathrm{ReS}_{0}$, but as it thereby becomes finitely axiomatisable, by a result of which many variants are found in the literature, and presumably going back to Bernays, we give it officially as that finite axiomatisation.

$\mathrm{DB}_{0}$ The system of which the set-theoretic axioms are Extensionality and the following nine set-existence axioms:

$$
\begin{array}{lll}
\varnothing \in V & \bigcup x \in V & a \cap\left\{(x, y)_{2} \mid x \in y\right\} \in V \\
\{x, y\} \in V & \operatorname{Dom}(x) \in V & \left\{(y, x, z)_{3} \mid(x, y, z)_{3} \in b\right\} \in V \\
x \backslash y \in V & x \times y \in V & \left\{(y, z, x)_{3} \mid(x, y, z)_{3} \in c\right\} \in V
\end{array}
$$

DB $\quad \mathrm{DB}_{0}$ plus $\Pi_{1}$ foundation.

1.8 REMARK All those nine are theorems of $\mathrm{ReS}_{0}+$ cartesian product, without foundation.

1.9 DeFinition We shall usually take the axiom of infinity in the form $\omega \in V$, though in one model that we consider, we must use a different formulation.

1.10 REMARK If we add the axiom of infinity plus the scheme of foundation for all classes to DB we obtain the system BS as formulated on page 36 of Devlin's book Constructibility:

BS $\operatorname{ReS}_{0}+$ Cartesian product + full foundation $+\omega \in V$.

\section{The Gandy-Jensen system}

The next system, called BST by Gandy, represents a considerable step forward, in that it involves the class of rudimentary functions. Foundation apart, it is finitely axiomatisable, and indeed needs only one axiom beyond those of $\mathrm{DB}_{0}$. We give first the scheme of Gandy, and in the next section shall see why all instances of it are derivable in the finitely-axiomatisable version.

$\mathrm{GJ}_{0} \quad \mathrm{~S}_{0}+$ the rudimentary replacement axiom:

$$
\forall x \exists w \overrightarrow{\forall v}: \in x \exists t: \in w \forall u(u \in t \Longleftrightarrow . u \in x \& \phi[u, \vec{v}]) .
$$

for $\phi$ any $\Delta_{0}$ formula.

1.11 REMARK At first glance, it might seem more appropriate to call that a collection axiom, since it says that a certain family of sets is included in a set, rather than being a set. But if $\varphi$ is $\Delta_{0}, x$ a set and $\vec{v}$ parameters, 
not necessarily in $x$, then a term $x_{1}$ and a $\Delta_{0}$ formula $\varphi_{1}$ are readily found so that $\mathrm{S}_{0}$ proves that $x_{1}$ is a set containing each parameter in the list $\vec{v}$, that $x \cap\{u \mid \varphi\}=x_{1} \cap\left\{u \mid \varphi_{1}\right\}$ and that the latter is a set. So $G_{0}$ indeed proves $\Delta_{0}$ separation.

1.12 REMARK $\mathrm{GJ}_{0}$ is the result of adding a single axiom, which I call $R_{8}$, to $\mathrm{DB}_{0}$ :

$$
\{x "\{w\} \mid w \in y\} \in V
$$

GJ $\quad \mathrm{GJ}_{0}+$ the scheme of $\Pi_{1}$ foundation.

\section{Flat restricted replacement}

The next system has what Gandy called the bounded replacement axiom, but we shall prefer to use the adjective "flat".

fReR $S_{0}$ plus the flat $\Delta_{0}$ replacement axiom: namely, for any $\phi$ in $\Delta_{0}$,

(Flat $\Delta_{0}$ Replacement)

$\forall x: \in u \exists ! y(\phi(x, y) \& y \subseteq z) \Longrightarrow \exists v \forall y[y \in v \Longleftrightarrow \exists x: \in u(\phi(x, y) \& y \subseteq z)]$.

In words, the image of a set by a function whose values are all included in a set is itself a set.

fReR $\quad \mathrm{fRe}_{0}+$ the scheme of $\Pi_{1}$ foundation.

\section{Flat restricted collection}

$\mathrm{fReC}_{0} \mathrm{~S}_{0}$ plus $\Delta_{0}$ separation plus the following scheme, for $\phi$ any $\Delta_{0}$ formula:

$$
\begin{aligned}
& \text { (Flat } \Delta_{0} \text { Collection) } \\
& \qquad \forall x: \in u \exists y(\phi(x, y) \& y \subseteq z) \Longrightarrow \exists v \forall x: \in u \exists y: \in v(\phi(x, y) \& y \subseteq z)] .
\end{aligned}
$$

$\mathrm{fReC} \quad \mathrm{fReC}_{0}+$ the scheme of $\Pi_{1}$ foundation.

1.13 REMARK $\Pi_{1}$ Foundation aside, the axioms of the above systems are all provable in the system $M_{0}$ studie $d$ in [M2], which is the system $\operatorname{ReS}_{0}+$ the power set axiom, $\mathcal{P}(x) \in V$ and is a subsystem of Mac Lane's system ZBQC, which in turn, shorn of the axiom of choice, is a subsystem of Zermelo's system Z.

\section{Restricted replacement}


We depart now from a linearly ordered set of systems: we shall see that $R e R$ is not a subsystem of $f R e C$, and I suspect that methods of Zarach will show that $\mathrm{fReC}$ is not a subsystem of ReR.

$\operatorname{ReR}_{0} \quad \mathrm{~S}_{0}+$ the following scheme, for $\phi$ any $\Delta_{0}$ formula:

$\left(\Delta_{0}\right.$ Replacement) $\forall x: \in u \exists ! y \phi(x, y) \Longrightarrow \exists v \forall y[y \in v \Longleftrightarrow \exists x: \in u \phi(x, y)]$.

ReR $\operatorname{ReR}_{0}+$ the scheme of $\Pi_{1}$ foundation.

\section{Kripke-Platek}

Finally we arrive at Kripke-Platek set theory, KP which we formulate with $\Pi_{1}$ foundation.

KP $\quad \Delta_{0}$ separation, $\Delta_{0}$ collection and $\Pi_{1}$ foundation.

$$
\left(\Delta_{0} \text { Collection }\right) \quad \forall x \exists y \phi \Longrightarrow \forall u \exists v \forall x: \in u \exists y: \in v \phi(x, y) .
$$

We shall indicate the addition of the axiom of infinity to one of the above systems by adding the letter I: thus $\mathrm{DB}_{0} \mathrm{I}, \mathrm{KPI}$.

1.14 REMARK By a result of Boffa, TCo, the statement that every set is a member of a transitive set, is not provable in Z, and therefore not in its subsystems. It is, however, provable in KP when that system is formulated, as here, to include $\Pi_{1}$ foundation, and in ReRI: see Proposition 2.108 and Problem 2.107.

\section{THEOREMS OF THE VARIOUS SYSTEMS}

\section{On ReS and finite sets:}

We shall work with two definitions of finite: we get an easy $\Sigma_{1}$ definition of $\mathbf{H F}$ by taking "finite" to mean "in bijection with a member of $\omega$ "; we shall get an easy proof that the union of two finite sets is finite by taking "finite" to mean "possesses a double well-ordering"; and we need $\Pi_{1}$ foundation to prove the equivalence of the two definitions (or to develop the arithmetic necessary if we wish only to work with the "member of $\omega$ " definition).

2.0 Definition $x$ is finite if $x$ carries a double well-ordering, that is, a linear ordering such that every non-empty subset has both a least and a greatest element.

2.1 Proposition (ReS) If a set is finite then it is in bijection with some member of $\omega$. 
Proof : Let $X$ be a set with a double well-ordering $\leqslant x$. We say that $f$ is an attempt at $x$ in $X$ if $\operatorname{Dom}(f)=\{y \mid y \leqslant x x\}$ and for all $y$ in $\operatorname{Dom}(f)$, $f(y)=\left\{f(z) \mid z<_{X} y\right\}$. The class

$$
\{x \mid x \in X \& \neg \exists f f \text { is an attempt at } x\}
$$

is $\Pi_{1}$ and if non-empty, has a $\leqslant X$-least element $\bar{x} . \bar{x}$ is not the first member of $X$, as an attempt at that point is easily built; nor can $\bar{x}$ be a successor, as an attempt at its predecessor is easily extended. So that class is in fact empty. Let $\varpi$ be an attempt at the largest element of $X$ : then a further induction shows that $\varpi$ maps $\left(X,<_{X}\right)$ bijectively to some finite ordinal.

As we are working without assuming that cartesian products exist in general, the converse, which is true, requires some preparation.

2.2 Lemma (ReS) For all $m$ and $k$ in $\omega,\{m\} \times k$ is a set.

Proof: Fix $m$. Use the fact that

$$
\left\{\left.(m, n)_{2}\right|_{n} n<k+1\right\}=\left\{\left.(m, n)_{2}\right|_{n} n<k\right\} \cup\left\{(m, k)_{2}\right\} .
$$

2.3 Lemma (ReS) For all $m$ and $k$ in $\omega, k \times\{m\}$ is a set.

Proof : Fix $m$. Use the fact that

$$
\left\{\left.(n, m)_{2}\right|_{n} n<k+1\right\}=\left\{\left.(n, m)_{2}\right|_{n} n<k\right\} \cup\left\{(k, m)_{2}\right\} .
$$

2.4 Proposition (ReS) For all $m$ and $n$ in $\omega, m \times n$ is a set.

Proof: Use the fact that

$$
(m+1) \times(m+1)=(m \times m) \cup(\{m\} \times m) \cup(m \times\{m\}) \cup(\{m\} \times\{m\}) . \dashv(2.4)
$$

2.5 REMARK Note that that cannot lead to a proof that $\omega \times \omega$ is a set. We cannot form the collection of attempts.

2.6 COROLlary (ReS) The cartesian product of two sets, each in bijection with a finite ordinal, is a set.

Proof : First, reason thus: if $g: m \longleftrightarrow a$ and $h: n \longleftrightarrow b$, define the function $f$ with domain $m \times n$ by

$$
f\left((i, j)_{2}\right)=(g(i), h(j))_{2} .
$$


Then the image of that function is $a \times b$.

But that reasoning, though sound in GJ, is not available in $B S$ or $\operatorname{ReS}_{0}$. Hence we must do an induction structured as above: first for $m=1$ prove, by induction on $n$, that for any $n$, and $g$ and $h$ as above, the cartesian product exists. Then do an induction on $m$.

2.7 REMARK In systems without the Axiom of Cartesian Products, it cannot be assumed that the inverse of an injective function will always exist: see the variant of Model 4.

2.8 Proposition (ReS) If $X$ is in bijection with some finite ordinal, then it is finite.

Proof : From the above we know that $X \times X$ and $n \times X$ exist. Now given $f: n \longleftrightarrow X$, we may form its inverse $g$ thus:

$$
g:=n \times X \cap\left\{\left.(a, b)_{2}\right|_{a, b}(b, a)_{2} \in f\right\}
$$

and we may then form the set $X \times X \cap\left\{\left.(x, y)_{2}\right|_{x, y} g(x) \leqslant g(y)\right\}$, which will be a double well-ordering.

2.9 Proposition Every subset of a finite set is finite.

Proof : a restriction of a double well-ordering is ditto.

2.10 Proposition If $x$ and $y$ are finite, so is $x \cup y$.

Proof : a double well-ordering of $x \cup y$ can easily be constructed given ones of $x$ and of $y \backslash x$.

2.11 LEMMA Let $z$ be a finite set, and $a \notin z$. Then $\left\{\left.y \cup\{a\}\right|_{y} y \in z\right\}$ is a set and is finite.

Proof : let $f: n \longleftrightarrow z$. Define

$$
\begin{aligned}
g(0) & =\{f(0) \cup\{a\}\} \\
g(k+1) & =g(k) \cup\{f(k) \cup\{a\}\}
\end{aligned}
$$

Then $g(n)$ will be defined - appeal to $\Pi_{1}$ foundation if not ! - and will be the desired set, which is evidently in bijection with $z$ and therefore finite.

2.12 LEMma $\left(\mathrm{S}_{0}\right)$ Let $z$ be a set, and $a \notin z$. Then $\mathcal{P}(z \cup\{a\})=\mathcal{P}(z) \cup$ $\left\{\left.y \cup\{a\}\right|_{y} y \in \mathcal{P}(z)\right\}$.

2.13 Proposition Let $w$ be finite. Then $\mathcal{P}(w)$ is a set and is finite.

Proof : write $F(a, z)$ for $\left\{\left.y \cup\{a\}\right|_{y} y \in z\right\}$. Let $f: n \longleftrightarrow w$. Define

$$
\begin{aligned}
g(0) & =\{\varnothing\} \\
g(k+1) & =g(k) \cup F(f(k), g(k))
\end{aligned}
$$


As before, we consider the least $m$ for which there is no attempt at $m$ for this recursion; and obtain a contradiction. So $g(n)$ will be the desired set $\mathcal{P}(w)$.

To see that $\mathcal{P}(w)$ is finite, argue, again by induction on $k \leq n$, and using 2.13, 2.14 and 2.15, that each $g(k)$ is finite, (the class of failures being again $\Pi_{1}$, the argument succeeds); so $g(n)$ is finite.

$\dashv(2.13)$

2.14 Proposition The cartesian product of two finite sets is finite.

Proof : by a similar argument, starting from the observation that $x \times(z \cup$ $\{a\})=(x \times z) \cup(x \times\{a\})$.

2.15 Proposition A surjective image of a finite set is finite.

[trivial if the surjection is a set; if it is defined by some formula, we may need full foundation.]

2.16 Definition $\mathcal{S}(x)={ }_{\mathrm{df}}\{y \mid y \subseteq x \& y$ is finite $\}$.

[It is not assumed that $\mathcal{S}(x)$ is a set.]

2.17 Definition Let $\Psi_{\mathcal{S}}(q, y)$ be the $\Delta_{0}$ formula $\varnothing \in q \& \forall w: \in q \forall x: \in$ $y w \cup\{x\} \in q$.

2.18 LEMMA $(\operatorname{ReS}) \Psi_{\mathcal{S}}(q, y) \Longrightarrow q \supseteq \mathcal{S}(y)$.

2.19 LEMma $(\operatorname{ReS}) \quad x \in \mathcal{S}(y) \Longleftrightarrow \exists f(x \subseteq y \& \exists n: \in \omega \operatorname{Fn}(f) \& f: n \longleftrightarrow$ $x)$. 1.6:

Hence, using the semi-suitablility of the constant $\omega$ recorded in Remark

2.20 COROLlaRY " $x \in \mathcal{S}(y)$ ” is $\Sigma_{1}^{\mathrm{ReS}}$.

2.21 LEMMA $(\operatorname{ReS}) \mathcal{S}(y) \in V \Longrightarrow \forall x\left[x \in \mathcal{S}(y) \Longleftrightarrow \forall q\left(\Psi_{\mathcal{S}}(q, y) \Longrightarrow x \in\right.\right.$ $q)$ ].

2.22 LEMMA $(\operatorname{ReS}) \mathcal{S}(y) \in V \Longrightarrow\left[z \subseteq \mathcal{S}(y) \Longleftrightarrow \forall q\left(\Psi_{\mathcal{S}}(q, y) \Longrightarrow q \supseteq z\right)\right]$.

2.23 LEMMA $(\operatorname{ReS}) \mathcal{S}(y) \in V \Longrightarrow\left[z=\mathcal{S}(y) \Longleftrightarrow z \subseteq \mathcal{S}(y) \& \Psi_{\mathcal{S}}(z, y)\right]$

Next, a principle of collection for finite sets.

2.24 Metatheorem Let $\mathfrak{A}$ be a $\Pi_{\mathfrak{k}}$ wff; then it is provable in $\operatorname{ReS}_{0}$ with $\Pi_{\mathfrak{k}+1}$ foundation that for $v$ finite, $\forall x: \in v \exists y \mathfrak{A} \Longrightarrow \exists w \forall x: \in v \exists y: \in w \mathfrak{A}$.

Proof : let $f: n \longleftrightarrow v$. Let $P(k)$ say that there is a function $g$ with domain $k$ such that $\forall i<k \mathfrak{A}(f(i), g(i))$. Find the least $k \leqslant n$ such that $P(k)$ fails. By taking cases on $k$, we see that it cannot exist. So $P(n)$ holds. Take the image of a corresponding $g$ for $w$.

2.25 REMARK The above result is self-strengthening to the case that $\mathfrak{A}$ is $\Sigma_{\mathfrak{k}+1}$. 


\section{Proof that HF models ZF minus infinity}

2.26 Definition We define $\mathbf{T F}$ to be the class of all finite transitive sets, and $\mathbf{H F}$ to be its union.

2.27 REMARK In a set theory without an axiom of foundation, HF might be strictly greater than $V_{\omega}$; for example, any Quine atom, that is, a set $x$ which equals its own singleton $\{x\}$, would be in $\mathbf{H F}$ as we have defined it. To exclude such ill-founded sets we should define $\mathbf{H F}$ as the union of transitive finite sets $u$ which are well-founded in the sense that $\forall x_{\subseteq u}(x \neq$ $\varnothing \Longrightarrow \exists y: \in x y \cap x=\varnothing$ ); and would then have to add occasional remarks to the discussion below. But as our chief focus is on contexts where the axiom of foundation is true, we may leave our definition of $\mathbf{H F}$ as it is.

2.28 METATHEOREM Let $\mathfrak{A}$ be any axiom of ZF other than that of infinity. Then $(\mathfrak{A})^{\mathbf{H F}}$ is a theorem of $\mathrm{ReS}_{0}+$ full foundation.

We begin a sequence of verifications. We frequently use the fact that for $\Delta_{0}$ concepts it suffices to prove that the object in question is in $\mathbf{H F}$ as its definition will relativise without difficulty.

2.29 LEMMA HF is transitive.

2.30 LeMma (Extensionality) ${ }^{\mathbf{H F}}$.

Proof : assured by the transitivity of $\mathbf{H F}$.

2.31 LEMMA $\mathbf{T F} \subseteq \mathbf{H F}$.

Proof : since $u$ transitive and finite implies $u \cup\{u\}$ is too; and hence $u$ is in $\mathbf{H F}$.

2.32 Corollary $(\mathrm{TCo})^{\mathbf{H F}}$.

2.33 LEMma (Emptyset) $)^{\mathbf{H F}}$

Proof: $\{\varnothing\}$ is transitive and finite.

2.34 LEMMA (Pairing) ${ }^{\mathbf{H F}}$

Proof : by Proposition 2.13 and the fact that the union of two transitive sets is transitive.

2.35 LEMMA (Sumset) ${ }^{\mathbf{H F}}$

Proof : if $x \in u \in \mathbf{T F}$, then $\bigcup x \subseteq u$ and $\bigcup x \in u \cup\{\bigcup x\} \in \mathbf{T F} . \quad \dashv(2.35)$

2.36 Lemma $\left(\Delta_{0} \text { Separation }\right)^{\mathbf{H F}}$

Proof : $\Delta_{0}$ separation will relativise to any transitive set.

2.37 REMARK Indeed an "external" version of $\Delta_{0}$ separation holds, in that $x \cap A \in \mathbf{H F}$ whenever $x \in \mathbf{H F}$ and $A$ is a $\Delta_{0}$ class, possibly with parameters that are not in $\mathbf{H F}$. 


\subsection{LEMMA (Powerset) $)^{\mathbf{H F}}$}

Proof : By Proposition 2.16 and the fact that if $u$ is transitive and $\forall x: \in$ $a x \subseteq u$ then $u \cup a$ is transitive.

2.39 LEMMA (set foundation) (Foundation) $)^{\mathbf{H F}}$

2.40 REMARK Foundation is definitely needed here: the result would be false if $\mathbf{H F}$ contained Quine atoms.

At this point we have proved that all of $M_{1}$ is true in $\mathbf{H F}$.

2.41 Definition $u^{\star}={ }_{\mathrm{df}} u \cup[u]^{1} \cup[u]^{2} \cup(u \times u)$.

2.42 LEMMA If $u$ is finite and transitive then so is $u^{\star}$.

Proof : $[u]^{1} \cup[u]^{2}$ is a $\Delta_{0}$ subclass of $\mathcal{P}(u), u \times u$ is finite by what we have seen, and the transitivity is easily verified.

2.43 Proposition "all sets are finite" is true in $\mathbf{H F}$.

Proof : if $x \in u \in \mathbf{T F}$ and $f: n \longleftrightarrow u$, then $f \subseteq u \times n ;(u \cup n)^{\star}$ is in TF, and so is $(u \cup n)^{\star} \cup u \times n \cup\{u \times n\}$.

2.44 LEMMA " $x \in \mathbf{H F}$ ” is $\Sigma_{1}^{\mathrm{ReS}}$.

Proof : $x \in \mathbf{H F} \Longleftrightarrow \exists u \exists f \exists n[n \in \omega \& \bigcup u \subseteq u \& f: n \longleftrightarrow u] . \quad \dashv(2.44)$

2.45 REMARK Here we benefit from the "simplified" definition of $\mathbf{H F}$ : if we had to say that $u$ is well-founded, that would introduce a $\Pi_{1}$ clause.

2.46 LEMMA (ReS) $\left(\left(\Pi_{1} \text { foundation }\right)\right)^{\mathbf{H F}}$.

Proof : Let $\Phi$ be $\Delta_{0}$ and $B=(\{x \mid \forall b \Phi\})^{\mathbf{H F}}$.

Let $C=\{x \mid \forall b[b \in \mathbf{H F} \Longrightarrow \Phi]\}$. Then $C$ is $\Pi_{1}$ and $B \subseteq C$; indeed $B=C \cap \mathbf{H F}$. Suppose that $B$ is non-empty and that $x$ is a member. Then there is $u \in \mathbf{T F}$ with $x \in u$. Then $C \cap u$ is $\Pi_{1}$ and non-empty; let $\bar{x}$ be a minimal element. Then $\bar{x}$ is a minimal element of $B$.

$\dashv(2.46)$

2.47 COROLlary $(\operatorname{ReS}) \quad\left(\Delta_{0} \text { collection }\right)^{\mathbf{H F}}$.

Thus ReS proves the relative consistency of the system MOST (as defined in [M2]) less infinity.

2.48 REMARK The above sheds some light on relative consistency strengths: reasoning in ReS we have shown the relative consistency of adding the power set axiom.

\section{With Full Foundation}

By results of [M3] we could now conclude that all of ZF minus infinity is true there provided we established the truth of the principle called Repcoll in [M3] and shown there to imply all the axioms of ZF in the system $M_{1}$, which is $\mathrm{M}_{0}+\mathrm{TCo}+$ set Foundation. $\mathrm{M}_{0}$ is $\operatorname{ReS}_{0}$ plus $\mathcal{P}(x) \in V$. 


\subsection{LEMma (ReS + full Foundation) (Repcoll $)^{\mathbf{H F}}$}

We shall not give the proof, because we shall derive the truth of ZF in HF by another route.

2.50 LEMMA Let $A$ be any class: then ReS + full Foundation proves $A \cap \mathbf{H F} \neq$ $\varnothing \Longrightarrow \exists x: \in A \cap \mathbf{H F} x \cap A=\varnothing$.

2.51 REMARK Here we definitely need the "simplied" version of $\mathbf{H F}$ that does not mention well-foundedness.

If we use full foundation we can establish an "external" form of full separation, as in the following scheme:

2.52 Lemma (ReS + full Foundation) $x \in \mathbf{H F} \Longrightarrow x \cap A \in \mathbf{H F}$ for $A$ any class.

Proof : let $f: n \longleftrightarrow x$. Consider the class

$$
B:=\left\{k \leqslant\left. n\right|_{k} \neg \exists y[y \subseteq x \& \forall m: \leqslant k(f(m) \in y \Longleftrightarrow f(m) \in A\} .\right.
$$

By full Foundation, that, if non-empty has a minimal element, $\bar{k}$, say. The case $\bar{k}=0$ is easily dismissed; if $\bar{k}=k+1$, we know that $z={ }_{\text {df }}\{f(i) \mid$ $i \leqslant k\} \cap A$ is a set, and $\{f(i) \mid i \leqslant \bar{k}\} \cap A$ will be either $z$ or $z \cup\{f(\bar{k})\}$; as both are sets, we have a contradiction; so the class $B$ is empty and the theorem is proved.

$\dashv(2.52)$

2.53 THEOREM (ReS + full Foundation) (full Collection) ${ }^{\mathbf{H F}}$.

Proof : from the above, since we know from Lemma 2.50 that HF models full foundation and from Proposition 2.44 that HF thinks that all sets are finite.

\section{With $\mathbf{H F} \in V$}

\subsection{Lemma $\left(\operatorname{ReS}_{0}+\mathbf{H F} \in V\right) \quad$ (Full Separation) ${ }^{\mathbf{H F}}$}

Proof : by re-writing the formula relativising all quantifiers to the set $\mathbf{H F}$, and then applying $\Delta_{0}$ Separation.

2.55 LEMMA ( $\operatorname{ReS}_{0}+\mathbf{H F} \in V+$ set foundation) (Full foundation) $\mathbf{H F}$

Proof : by Lemma 2.54 and Corollary 2.32 .

Another example of the amount of foundation needed for a proof being reduced by the assumption that $\mathbf{H F} \in V$ is furnished by the next subsection. 


\section{Do graphs of recursive functions exist ?}

2.56 Consider the following argument, intended to prove that addition on $\omega$ is total:

Let $\phi(m, n)$ say that there is no function with domain $(m+1) \times(n+1)$ which satisfies the definition of addition for $m^{\prime}+n^{\prime}$ for $m^{\prime} \leqslant m$ and $n^{\prime} \leqslant n$. [We call such functions attempts at integer addition.]

Consider the class of $m \in \omega$ such that there is some $n \in \omega$ for which $\phi(m, n)$ is true. If non-empty, use $\Pi_{1}$ foundation to find its least member, $\bar{m}$, which cannot be 0 , as the function $f(0, n) \equiv n$ would work: a subset of $(n+1) \times(\{0\} \times(n+1))$, and so is some $m+1$. Now minimise $n$. Again it cannot be 0 . So it is some $n+1$. But we have a function $h$ defined up to $m+1, n$, and can extend it to $g$ by setting $g(m+1, n+1)=h(m+1, n)+1$, a contradiction. We have proved the following:

2.57 Proposition (ReS) Every pair $(m, n)$ of integers is in the domain of some attempt at integer addition.

2.58 REMARK " $f$ is an attempt at addition" is $\Delta_{0}$, and therefore rudimentary.

2.59 Now comes the great task of putting all the attempts together: what does it take to prove that the graph of integer addition is a set? The axiom of infinity is certainly necessary, but not sufficient: we shall see that fReR would do this very well, and that neither $\operatorname{ReS}_{0}$, BS nor GJ can do it. Happily, our system DS does prove it. $\mathbf{H F} \in V$ would also do it.

\section{On $\mathrm{DB}_{0}$ l:}

2.60 Proposition ( $\left.\mathrm{DB}_{0} \mathrm{l}\right)[\omega]^{1}$ and $[\omega]^{2}$ exist.

Proof : $\omega \in V$ is an axiom of $\mathrm{DB}_{0} \mathrm{l}$. By the definition of ordered pair, $[\omega]^{1} \cup[\omega]^{2} \subseteq \bigcup(\omega \times \omega)$, and the result follows by $\Delta_{0}$ separation. $\quad \dashv(2.60)$

\section{On GJ and the class of rudimentary functions}

The companion papers Rudimentary recursion and Rudimentary forcing will contain more detailed material on rudimentary functions and related topics. Here we merely give a summary, drawing on but in places differing from the material in Jensen [J2], Gandy [G], Devlin [Dev] and Dodd [Do].

2.61 Corresponding to the systems of $\mathrm{DB}_{0}$ and $\mathrm{GJ} \mathrm{J}_{0}$, we introduce the rudimentary functions $R_{0}, \ldots R_{8}$ and certain auxiliary functions $A_{0} \ldots A_{15}$ generated by them: this is not the shortest possible list, but one that conveniently extends the list that generates the $\Delta_{0}$ separators. Of the auxiliaries, we list only the most important, $A_{14}$. 


$$
\begin{aligned}
& R_{0}(x, y)=\{x, y\} \\
& R_{1}(x, y)=x \backslash y \\
& R_{2}(x)=\bigcup x \\
& R_{3}(x)=\operatorname{Dom}(x) \\
& R_{4}(x, y)=x \times y \\
& R_{5}(x)=x \cap\left\{(a, b)_{2} \mid a \in b\right\} \\
& R_{6}(x)=\left\{(b, a, c)_{3} \mid(a, b, c)_{3} \in x\right\} \\
& R_{7}(x)=\left\{(b, c, a)_{3} \mid(a, b, c)_{3} \in x\right\} \\
& \quad A_{14}(x, y)=x "\{y\}\left[=\operatorname{Dom}\left((x \cap([\bigcup \bigcup x] \times\{y\}))^{-1}\right)\right] \\
& R_{8}(x, y)=\left\{x^{\prime \prime}\{w\} \mid w \in y\right\}
\end{aligned}
$$

2.62 Proposition Each of $R_{0} \ldots R_{7}$ and $A_{0}, \ldots A_{14}$ is $\mathrm{DB}_{0}$-suitable; $R_{8}$ is $\mathrm{GJ}_{0}$-suitable.

2.63 Definition Let $\mathcal{B}$ be the closure of $R_{0} \ldots R_{7}$ under composition.

2.64 Proposition Each function in $\mathcal{B}$ is $\mathrm{DB}_{0}$-suitable.

2.65 Proposition For each $\Delta_{0}$ class $A$ the map $x \mapsto x \cap A$ is in $\mathcal{B}$.

2.66 REMARK That corresponds to the derivability of $\Delta_{0}$ separation in $\mathrm{DB}_{0}$.

2.67 Definition Let $\mathcal{R}$ be the closure of $R_{0} \ldots R_{8}$ under composition.

2.68 Proposition Each function in $\mathcal{R}$ is $\mathrm{GJ}_{0}$-suitable.

The collection of functions in $\mathcal{R}$ is also closed under formation of images: by which is meant that if $F$ is in $\mathcal{R}$ so is $x \mapsto F$ " $x$. To prove this we introduce the notion of a companion. We will actually have two such notions.

Let T be some system of set theory extending DB, and let $G$ and $F$ be $\Delta_{0}$ classes such that T proves that both $G$ and $F$ are total functions.

2.69 Definition $G$ is a 1-companion of $F$ in $\mathrm{T}$ if $G$ is T-suitable and

$$
\vdash_{\mathrm{T}} \vec{x} \in \vec{u} \Longrightarrow F(\vec{x}) \downarrow \in G(\vec{u})
$$

2.70 Definition $H$ is a 2-companion of $F$ in $\mathrm{T}$ if $H$ is T-suitable and

$$
\vdash_{\mathrm{T}} \vec{x} \in \vec{u} \Longrightarrow F(\vec{x}) \downarrow \subseteq H(\vec{u})
$$

where $\vec{x} \in \vec{u}$ abbreviates $x_{1} \in u_{1} \& \ldots x_{n} \in u_{n}$ for an appropriate $n$.

The collection of functions with a 1-companion is easily seen to be closed under composition; but usually it is much easier to spot a 2-companion of a function. The following is easily verified by inspection. 
2.71 Proposition Each of the functions $R_{0}, \ldots, R_{7}$ and $A_{14}$ has a 2 companion in $\mathrm{DB}_{0}$.

\section{Generation of 1-companions from 2-companions and separators.}

The Gandy-Jensen Lemma is the core of the proof that $\mathcal{R}$ is closed under formation of images. Versions of it are to be found in the papers of Gandy [G] and Jensen [J1]. We discuss it only for 1-ary functions.

2.72 The Gandy-Jensen Lemma Suppose that $H$ is a 2-companion of $F$, and that ' $a \in F(b)$ ' is $\Delta_{0}$. Then $F$ is generated by composition from $H$ and members of $\mathcal{B}$; further $F$ " $x \in V$ and $F$ " (as a function) is generated by $H$ and members of $\mathcal{R}$ and (as a term) is $\mathcal{S}$-suitable and is a 1-companion of $F$ in $\mathcal{S}$.

Proof : We have

$$
x \in u \Longrightarrow F(x) \subseteq H(u) .
$$

Form

$$
h(u)=_{\mathrm{df}}(H(u) \times u) \cap\left\{(a, b)_{2} \mid b \in u \& a \in F(b)\right\} .
$$

Actually, we could just take

$$
h(u)=_{\mathrm{df}}(H(u) \times u) \cap\left\{(a, b)_{2} \mid a \in F(b)\right\} .
$$

Since $a \in F(b)$ is $\Delta_{0}$ and for each $\Delta_{0} A$, the separator $x \mapsto x \cap A$ is in $\mathcal{F}$ and is $D B$-suitable, we have that $h$ is generated by $H$ and functions in $\mathcal{F}$.

Now note that for $b \in u, F(b)=h(u)$ " $\{b\}=A_{13}(h(u), b)$, so $F$ is built from $H$ and functions in $\mathcal{F}$; if $R_{8}$ is available, we may argue further that $F^{\prime \prime} u=R_{8}(h(u), u)$ so $F$ " is built from $H$ and rudimentary functions; hence $F$ " $u \in V$, and this function $F$ " now forms a 1-companion of $F . \quad \dashv(2.72)$

Proofs that $\mathcal{R}$ is closed under the rudimentary schemata may be found in the cited works on fine structure.

\section{A single generating function for $\operatorname{rud}(u)$}

Following Jensen, we define $\operatorname{rud}(u)$ to be the rud closure of $u \cup\{u\}$. Various functions with properties similar to those of the following may be found in the literature.

\subsection{DEFINITION}

$$
\begin{aligned}
\mathbb{T}(u)= & u \cup\{u\} \\
& \cup[u]^{1} \cup[u]^{2}
\end{aligned}
$$




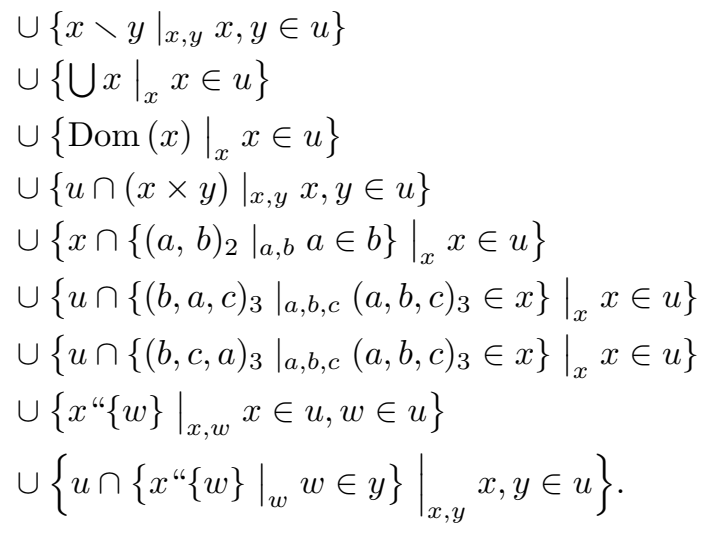

2.74 REMARK The successive lines of the definition of $\mathbb{T}$, after the first, may be written more prosaically as $R_{0}$ " $(u \times u), R_{1}$ " $(u \times u), R_{2}$ " $u, R_{3}$ " $u$, $\left\{\left.u \cap R_{4}(x, y)\right|_{x, y} x, y \in u\right\}, R_{5}$ " $u,\left\{\left.u \cap R_{6}(x)\right|_{x} x \in u\right\},\left\{\left.u \cap R_{7}(x)\right|_{x} x \in\right.$ $u\}, A_{14} "(u \times u)$ and $\left\{\left.u \cap R_{8}(x, y)\right|_{x, y} x, y \in u\right\}$. It will be notationally convenient to treat all these functions as having three variables, so let us define $S_{i}(u ; x, y):=R_{i}(x, y)$ for $i=0,1 ; S_{i}(u ; x, y):=R_{i}(x)$ for $i=2,3,5$; $S_{i}(u ; x, y):=u \cap R_{i}(x, y)$ for $i=4,8 ; S_{i}(u ; x, y):=u \cap R_{i}(x)$ for $i=6,7$; and $S_{9}(u ; x, y):=A_{14}(x, y)$.

Then each line is of the form $S_{i}$ " $\{\{u\} \times(u \times u))$ for some $i$. We have proved the first clause of the following, and the others are easy.

2.75 Proposition $\mathbb{T}$ is rudimentary, $u \subseteq \mathbb{T}(u)$ and $u \in \mathbb{T}(u)$. Further, if $u$ is transitive, then $\mathbb{T}(u)$ is a set of subsets of $u$, and hence $\mathbb{T}(u)$ is transitive.

2.76 REMARK It will not in general be true that $u \subseteq v \Longrightarrow \mathbb{T}(u) \subseteq \mathbb{T}(v)$, the problem being that $u \in \mathbb{T}(u)$, but if $v$ is countably infinite, so is $\mathbb{T}(v)$ which therefore cannot contain all the subsets of $v$. Fortunately, $u \subseteq \mathbb{T}(u) \subseteq$ $\mathbb{T}^{2}(u) \ldots$

2.77 Lemma For $x, y$ in $u, R_{4}(x, y)=x \times y \subseteq u \times u \subseteq \mathbb{T}^{2}(u)$.

2.78 Corollary For $x, y$ in $u, R_{4}(x, y) \in \mathbb{T}^{3}(u)$.

2.79 Lemma For $a, b c$ in $u,(a, c)_{2} \in \mathbb{T}^{2}(u)$ and $(b, a, c)_{3} \in \mathbb{T}^{4}(u)$.

2.80 Corollary For $x \in u, R_{6}(x)$ and $R_{7}(x)$ are in $\mathbb{T}^{5}(u)$.

2.81 LemMa For $x, y \in u, R_{8}(x, y) \in \mathbb{T}^{2}(u)$.

Proof: For $x, w$ in $u, x " w \in \mathbb{T}(u)$, so $R_{8}(x, y)=\mathbb{T}(u) \cap\left\{x " w \mid{ }_{w} w \in y\right\}$; $x, y \in \mathbb{T}(u)$, so $R_{8}(x, y) \in \mathbb{T}^{2}(u)$.

2.82 Proposition For any transitive $u, \bigcup_{n \in \omega} \mathbb{T}^{n}(u)$ is the rudimentary closure of $u \cup\{u\}$, and in it, TCo holds. 
2.83 Problem I do not see how to form a single rud function which will in similar fashion give the rud closure of $u$. Perhaps this has something to do with the question of MacAloon and Stanley discussed in $\S 14$.

\section{Other remarks on GJ}

2.84 REMARK RR produces a collection of subsets of $x$.

2.85 Proposition (Gandy; Jensen) A transitive set is rud closed (= basically closed) iff it models $\mathrm{GJ}_{0}$.

2.86 REMARK $\mathrm{GJ}_{0}$ proves that the cartesian product of two sets is a set.

2.87 REMARK $\Delta_{0}$ separation is a theorem scheme of $\mathrm{GJ}_{0}$.

2.88 Proposition $R R$ is self-strengthening to

$\left(R R^{+}\right) \quad \forall x_{1} \forall x_{2} \exists w \forall \vec{v}: \in x_{1} \exists t: \in w \forall u\left(u \in t \Longleftrightarrow . u \in x_{2} \& \phi[u, \vec{v}]\right)$.

for $\phi$ any $\Delta_{0}$ formula.

2.89 Problem Does GJ prove the existence of a bijection between $\omega$ and $\omega \times \omega ?$

I suspect that BS does, as everything necessary is in HF.

The next result is a scheme of theorems:

2.90 Proposition $\left(G J_{0}\right)$ Each $[\omega]^{\mathfrak{k}}$ exists; indeed, each $[a]^{\mathfrak{k}}$ exists for any set $a$.

Proof : $[a]^{0}=\{\varnothing\} \in V .[a]^{1}=A_{0}$ " $a \in V .[a]^{\mathfrak{k}+1}=\left\{s \cup\{x\} \mid(s, x)_{2} \in\right.$ $\left.\left([a]^{\mathfrak{k}} \times a\right) \cap\left\{(s, x)_{2} \mid x \notin s\right\}\right\}$, which is in $V$, being of the form $h^{\prime \prime} b$ for some set $b$ and rudimentary function $h$.

2.91 Theorem (GJ) $\forall a \forall k: \in \omega[a]^{k} \in V$.

2.92 Problem Is the quantified form provable without $\Pi_{1}$ foundation?

2.93 TheOREM (GJ) $\forall a \forall m: \in \omega^{m} a \in V$.

Proof : Fix $a$, and consider the $\Pi_{1}$ class

$$
\begin{aligned}
\omega \cap\{m \mid \neg \exists x[\forall y: \in x(y: m \longrightarrow a \\
\& \forall k: \in m \forall t: \in a \exists z: \in x(z\lceil k=y\lceil k \& z(k)=t))]\} .
\end{aligned}
$$

The theorem states that that class is empty: if it is not, let $m$ be its minimal element. But then $m$ is either 0 or a successor; if 0 , nothing to 
prove; if $m=k+1$, then ${ }^{k} a$ exists and we can then form ${ }^{m} a$ as the image of a rudimentary function applied to ${ }^{k} a \times a$, since

$$
{ }^{k+1} a=\left\{\left.f \cup\left\{(t, k)_{2}\right\}\right|_{f, t} f \in{ }^{k} a \& t \in a\right\} .
$$

2.94 Problem Is ${ }^{m} a$ suitable in any sense ? What seems to be true is that each ${ }^{\mathfrak{k}} a$ is rud, and each $[a]^{\mathfrak{k}}$ but that $[b]^{n}$ is not a rud function of two variables, as if it were, $\mathcal{S}(b, x)={ }_{\mathrm{df}} \bigcup_{n \in x}[b]^{n}$ would be a rud function; but by Gandy the rud closure of $\omega+1$ omits $\mathcal{S}(\omega)=\mathcal{S}(\omega, \omega)$.

\section{On fReR}

That GJ is a subsystem of fReR would follow from the theory of companions.

2.95 Proposition (fReRI) The graph of addition, and indeed of every primitive recursive function is a set.

Proof: we prove first that $\forall n \exists f f \subseteq \omega \times(\omega \times \omega)$ with $\operatorname{Dom}(f)=n \times n$ and

$$
\forall m:<n \forall k:<n[f(m, 0)=m \& f(m, k \dot{+} 1)=f(m, k) \dot{+} 1] .
$$

The collection of all such f's is a set, of which the union will be the graph of addition.

2.96 CoROLlaRY The Ackermann relation may be proved to exist in fReR.

2.97 CoRollaRY (fReRI) $\mathcal{S}(\omega) \in V$.

For another proof, one may reflect that every finite set of natural numbers is of the form

$$
\left\{i \mid \mathrm{p}_{i} \text { divides } n\right\}
$$

for some $n$, where $\mathrm{p}_{i}$ is the $i^{\text {th }}$ rational prime.

2.98 Corollary (fReRI) Even is a set.

2.99 Proposition (fReRI) If $x$ is countable then $\mathcal{S}(x)$ exists.

2.100 Problem Does fReR prove that each $\mathcal{S}(x)$ is a set ? or at least that each $\mathcal{S}(\zeta)$ exists ?

It may be that in a model with amorphous sets in the sense of Truss, there will be difficulties.

2.101 Proposition fReR is self-strengthening to allowing $\phi$ in (BdR) to have further free variables.

Proof: Note that if $\operatorname{Rel}(s)$ and Dom $s \neq \varnothing$ and $s \subseteq z \times\{w\}$, then $s=y \times\{w\}$ for some $y \subseteq z$; further, $\operatorname{Dom} s=\{w\}, \bigcup \operatorname{Dom} s=w$ and $\operatorname{Im} s=y$. 
Let $\psi(x, s) \Longleftrightarrow \Longleftrightarrow_{\mathrm{df}} \operatorname{Rel}(s) \& \operatorname{Dom} s \neq \varnothing \& \phi(x, \operatorname{Im} s, \bigcup \operatorname{Dom} s)$. Then $\psi$ is $\Delta_{0}$. Let $z_{1}=z \times\{w\}$, and suppose that $\forall x: \in u \exists ! y[\phi(x, y, w) \& y \subseteq z$. $]$ That tells us that

$$
\forall x: \in u \exists ! s\left[\psi(x, s) \& s \subseteq z_{1}\right]
$$

so applying (BdR), we deduce that the class

$$
\{y \times\{w\} \mid \exists x: \in u \phi(x, y, w) \& y \subseteq z\}
$$

is a set, $v$, say. Then applying an appropriate rudimentary function, we see that the class $\{\operatorname{Im} t \mid t \in v\}$ is a set; but that class is $\{y \mid \exists x: \in$ $u \phi(x, y, w) \& y \subseteq z\}$, as desired.

$\dashv(2.101)$

\section{On ReRI}

\subsection{Proposition (ReRI) $\omega+\omega \in V$.}

Proof : $\forall n: \in \omega \exists f[\operatorname{Fn}(f) \& \operatorname{Dom}(f)=n+1 \&(f(0)=\omega) \& \forall m:<$ $n f(m \dot{+})=f(m) \dot{+} 1]$, by an easy application of $\Pi_{1}$ foundation, and for each $n$ there cannot be two distinct such $f$ 's. Hence by $\Delta_{0}$ replacement, the set $F$ of those $f$ 's exists, and $\omega+\omega$ will be $\operatorname{Im}(\bigcup F)$.

\subsection{Proposition (ReRI) $\mathcal{S}(x) \in V$}

Proof : Fix $x$. Let $G$ be the rudimentary function given by $G(y, z)=\{a \cup b \mid$ $\left.a \in y \& b \in[z]^{1}\right\}$. We seek to define a function $f: \omega \longrightarrow V$ by the following recursion:

$$
f(0)=[x]^{1} ; f(n+1)=G(f(n), x) .
$$

We call $f$ an attempt at $n$ if

$$
F n(f) \& \operatorname{Dom}(f)=n+1 \& f(0)=[x]^{1} \& \forall k: \in n f(k+1)=G(f(k), x) .
$$

Using set foundation it is easily seen that any two attempts agree on their common domain, so that there is at most one attempt at $n$; and, using $\Pi_{1}$ foundation to obtain a minimal element of the class of those $n$ at which there is no attempt, we see that that class in fact must be empty, and hence that there is a unique attempt at each $n$.

Since being an attempt is $\Delta_{0}$ in our present system, ReRI proves that there is a set containing (exactly) the attempts for each $n$. The union of that set is therefore a set and a function, and the union of its image is $\mathcal{S}(x)$.

2.104 REMARK A similar argument will show in ReRI that the transitive closure of any set exists. 


\subsection{Proposition (ReRI) HF $\in V$}

We omit the proof as the Proposition is a special case of Proposition 8.28 .

2.106 REMARK I would guess that ReRI suffices to define the relation $u \models \varphi$, and the constructible hierarchy; and that the $L$ of a model of ReRI is a model of KPI, so that indeed the two theories are equiconsistent.

\subsection{PROBLEM Does ReR prove TCo?}

\section{On KP}

\subsection{PROPOSITION (KP) TCo}

Proof: Let $A=\{x \mid \forall u \bigcup u \subseteq u \Longrightarrow x \notin u\}$. By $\Pi_{1}$ foundation, $A$, if non-empty, has an $\in$-minimal element $\bar{x}$. So $\forall x: \in \bar{x} \exists u \bigcup u \subseteq u \& x \in u$. By $\Delta_{0}$ Collection there is a $v$ such that $\forall x: \in \bar{x} \exists u: \in v \bigcup u \subseteq u$ \& $x \in u$. Let $w=v \cap\{u \mid \bigcup u \subseteq u\}$. $w$ is a set by $\Delta_{0}$ separation; let $\bar{u}=\bigcup w$. The $\bar{u}$ is transitive and $\bar{x} \subseteq \bar{u}$. Hence $\bar{x}$ is a member of the transitive set $\bar{u} \cup\{\bar{x}\}$, and is therefore in $\bar{A}$, a contradiction.

\section{REMARKS ON TRANSITIVE MODELS}

Many of our models are of the following simple kind. We define a class A of transitive sets, and take $\mathbf{M}=\bigcup \mathbf{A}$.

3.0 Proposition i) Such an $\mathbf{M}$ will always be transitive, and will model the Axiom of Extensionality and the full scheme of Foundation for all classes, and be absolute for all $\Delta_{0}$ formulæ.

ii) If $\mathbf{A}$ is non-empty, the axiom $\varnothing \in V$ will be true in $\mathbf{M}$; if $\omega+1 \in \mathbf{A}$ then $\mathbf{M}$ will model $\omega \in V$.

iii) If $u \in \mathbf{A}$ and $y \subseteq u$ implies $u \cup\{y\} \in \mathbf{A}$, then $\mathbf{M}$ will model the sumset axiom; further $\mathbf{M}$ will be supertransitive and will therefore model the full separation scheme; and $\mathbf{A}$ will be a subclass of $\mathbf{M}$, which will therefore model TCo, and indeed the transitive closure of any member of $\mathbf{M}$ will also be a member of $\mathbf{M}$.

iv) If the hypothesis of (iii) holds and, additionally, $u \in \mathbf{A}$ and $v \in \mathbf{A}$ implies $u \cup v \in \mathbf{A}$, then $\mathbf{M}$ will model AxPair.

The proof is straightforward. Models of that kind, therefore, are always models of Gandy's system $\operatorname{ReS}_{0}$ with TCo, and with full foundation and full separation. 
3.1 REMARK Just to clarify that last remark: to prove full foundation in the model, we require (if the model be a proper class) full foundation in the background theory; and similarly for full separation.

\section{Slim models of weak systems}

Many such classes $\mathbf{A}$ can be found by modifying a definition to be found in Slim Models of Zermelo Set Theory:

3.2 Definition $\mathcal{T}$ is weakly fruitful if

(i) every $x$ in $\mathcal{T}$ is transitive;

(iii) $x \in \mathcal{T} \& y \in \mathcal{T} \Longrightarrow x \cup y \in \mathcal{T}$;

(iv') $x \in \mathcal{T} \& a \subseteq x \Longrightarrow x \cup\{a\} \in \mathcal{T}$.

The missing condition (ii) lists three possible conditions on the ordinals in the class $\mathcal{T}$ :

(ii) $1 \in \mathcal{T} ; \omega+1 \in \mathcal{T} ; O N \subseteq \mathcal{T}$, respectively;

So our theorem above gives the following:

3.3 Proposition If $\mathcal{T}$ is weakly fruitful, then $\bigcup \mathcal{T}$ will be a supertransitive model of $\operatorname{ReS}_{0}$ with TCo, full separation and full foundation, and if $1 \in \mathcal{T}$, of Empty Set; if $\omega+1 \in \mathcal{T}$, the axiom of infinity will hold in $\bigcup \mathcal{T}$ in the form $\omega \in V$, and in the third case, the model $\bigcup \mathcal{T}$ will contain all ordinals.

There is a simple further requirement on $\mathbf{A}$ that ensures that $\bigcup \mathbf{A}$ is closed under cartesian products. Recall our definition from section 2:

DEFINITION $u^{\star}={ }_{\mathrm{df}} u \cup[u]^{1} \cup[u]^{2} \cup(u \times u)$.

3.4 LEMMA $u^{\star}$ is BS suitable; if $u$ is transitive, so is $u^{\star}$, and $u \times u \subseteq u^{\star}$.

3.5 Proposition If $\mathbf{A}$ is a collection of transitive sets closed under ${ }^{\star}$, union of two elements, adding a subset to an element, and containing interesting ordinals, then $\bigcup \mathbf{A}$ will model BS with TCo, full foundation and full separation.

As in Slim Models, we may obtain some interesting examples of such models by estimating the rate of growth of various transitive sets. Given a function $Q: \omega \longrightarrow V$, set $f_{x}^{Q}(n)=\overline{\overline{x \cap Q(n)}}$. For $\mathcal{G}$ a class of functions, form $\mathcal{T}^{Q, \mathcal{G}}=_{\mathrm{df}}\left\{x \mid \bigcup x \subseteq x \& f_{x}^{Q} \in \mathcal{G}\right\}$.

3.6 Proposition If $\mathcal{G}$ has these properties then $\mathcal{T}^{Q, \mathcal{G}}$ will be weakly fruitful:

$$
\begin{aligned}
& f \leqslant g \in \mathcal{G} \Longrightarrow f \in \mathcal{G} ; \\
& f, g \in \mathcal{G} \Longrightarrow f+g \in \mathcal{G} ;
\end{aligned}
$$


$\bigcup x \subseteq x \& f_{x}^{Q} \in \mathcal{G} \Longrightarrow f_{x}^{Q}+1 \in \mathcal{G}$.

The three conditions on ordinals considered correspond to the three requirements

$$
f_{1}^{Q} \in \mathcal{G} ; f_{\omega+1}^{Q} \in \mathcal{G} ; \forall \zeta f_{\zeta}^{Q} \in \mathcal{G} .
$$

3.7 Proposition A sufficient further condition on $\mathcal{G}$ for cartesian products to exist in $\bigcup \mathcal{T}^{Q, \mathcal{G}}$, when $Q(n)=V_{n}$, is this:

$$
(f \in \mathcal{G} \& g \in \mathcal{G} \& C \in \omega) \Longrightarrow C . f . g \in \mathcal{G}
$$

Proof: We must show that in these circumstances, $u \in \mathcal{T} \Longrightarrow u^{\star} \in \mathcal{T}$. Note that for $n \geqslant 2$,

$\overline{\overline{[u]^{1} \cap V_{n}}}=\overline{\overline{u \cap V_{n-1}}} ; \overline{\overline{[u]^{2} \cap V_{n}}} \leqslant\left(\overline{\overline{u \cap V_{n-1}}}\right)^{2} ; \overline{\overline{(u \times u) \cap V_{n}}}=\left(\overline{\overline{u \cap V_{n-2}}}\right)^{2}$.

Hence $f_{u^{\star}}^{Q}(n)=\overline{\overline{u^{\star} \cap V_{n}}} \leqslant f_{u}^{Q}(n)+f_{u}^{Q}(n-1)+\left(f_{u}^{Q}(n-1)\right)^{2}+\left(f_{u}^{Q}(n-\right.$ 2) $)^{2}$. Since $V_{n} \subseteq V_{n+1}$ each $f_{u}^{Q}$ is monotonic; the proposition now follows by elementary analysis.

Of our collection, Models 3, 5 and 8 are obtained by the above rateof-growth method, of which the last two model the Axiom of Cartesian Products. Models 1, 2, 4, 6, 7, 9, and 10 are obtained by a different method, which we now describe.

3.8 Proposition Let $X$ be a class. Put $\mathbf{A}^{X}=$ the class of those transitive $u$ whose intersection with $X$ is finite. Then $\mathbf{M}^{X}={ }_{\mathrm{df}} \cup \mathbf{A}^{X}$ will be supertransitive and will model extensionality; foundation; full separation, difference and $\bigcup$; pairing; and $\mathrm{TCo}$, since $\mathbf{A}^{X} \subseteq \mathbf{M}^{X}$; as long as $X$ contains only finitely many ordinals, $\mathbf{M}^{X}$ will model infinity; if $u$ in $\mathbf{A}^{X}$ implies $u^{\star}$ is in $\mathbf{A}^{X}$ then $\mathbf{M}^{X}$ will be closed under cartesian products.

Models 11-15 are obtained by yet other methods. TCo holds in all these models; all are supertransitive save for Model 14 and some variants of Model 11.

\section{MODELS OF ReS}

\section{Gandy: A set which models PZ but not BST.}

We take $\mathbf{G}_{1}$ to be the class of all $x$ such that everything in $t c l(\{x\})$ is either finite or differs from $\omega$ by a finite set. Gandy remarks that (a) $\mathbf{G}_{1}$ is transitive; (b) if $x$ is in $\mathbf{G}_{1} \bigcup x$ is a subset of $\mathbf{G}_{1}$; (c) $\omega \in \mathbf{G}_{1}$; (d) $\mathbf{G}_{1}$ contains every finite subset of itself, and every $x$ in $\mathbf{G}_{1}$ is a substitutable 
constant in his sense. (e) $\mathbf{G}_{1}$ satisfies $\Delta_{0}$ separation, the proof of which uses the fact that every $\Delta_{0}$ subset of $\omega$ is finite or cofinite, by his quantifier elimination lemma. (f) $\omega \times \omega$ is not in $A$.

It follows from those remarks that $\mathbf{G}_{1}$ is not supertransitive and that $\mathbf{G}_{1} \cap O N=\omega+\omega$. We verify the following in detail:

4.0 Proposition If $x \in \mathbf{G}_{1}$ then so are $\bigcup x$ and $\operatorname{tcl}(x)$.

Now $\operatorname{tcl}(\{\bigcup x\})=\{\bigcup x\} \cup \operatorname{tcl}(\bigcup x)$ and $\operatorname{tcl}(\bigcup x) \subseteq \operatorname{tcl}(x) \subseteq \operatorname{tcl}(\{x\})$, so it is enough to prove that if $x$ is in $\mathbf{G}_{1}, \bigcup x$ is either finite or almost $\omega$.

First note that if $x$ is finite and in $\mathbf{G}_{1}$, then $x=y \cup z$, where $y$ is the set of finite members of $x$ and $z$ is the set of members of $x$ which are infinite and therefore almost equal to $\omega$. If $z$ is empty, then $\bigcup x=\bigcup y$, and is thus finite. If $z$ is non-empty, then $\bigcup x=\bigcup y \cup \bigcup z ; y$ and $z$ are both finite, and so $\bigcup y$ will be finite, and $\bigcup z$ will be almost equal to $\omega$. Hence $\bigcup x$ is almost equal to $\omega$.

Thus we have verified that if $x$ is a finite member of $\mathbf{G}_{1}$ then $\bigcup x \in \mathbf{G}_{1}$.

If on the other hand, $x$ almost equals $\omega$, then we can write $x=y \cup z$ where $z$ is a cofinite subset of $\omega$, and $y$ is a finite set disjoint from $\omega=\varnothing$. As $\mathbf{G}_{1}$ is transitive, $y$ is a a finite subset of it, and therefore a member of it, and therefore $\bigcup y \in \mathbf{G}_{1}$, by the previous paragraph. So $\bigcup x=\bigcup y \cup \omega$; $\bigcup y$ is either finite or almost $\omega$; either way, $\bigcup x$ is almost $\omega$.

To show that $x \in \mathbf{G}_{1} \Longrightarrow \operatorname{tcl}(x) \in \mathbf{G}_{1}$, suppose that $x$ is a counterexample of minimal rank. It is enough to show that $\operatorname{tcl}(x)$ is either finite or almost $\omega$.

$$
\operatorname{tcl}(x)=x \cup \bigcup_{t \in x} \operatorname{tcl}(t),
$$

where by the minimality of $x \operatorname{each} \operatorname{tcl}(t)$ is in $\mathbf{G}_{1}$.

4.1 REMARK The displayed formula implies easily that $\operatorname{tcl}(a \cup b)=\operatorname{tcl}(a) \cup$ $\operatorname{tcl}(b)$.

So if $x$ is finite, $\operatorname{tcl}(x)$ is the union of a finite set and finitely many sets each either finite or almost $\omega$, so that $\operatorname{tcl}(x)$ itself must be either finite or almost $\omega$, and therefore in $\mathbf{G}_{1}$. Thus the minimal counterexample must be almost $\omega$.

But now we may write $x$ as the union of a finite set $y$ disjoint from $\omega$ and a cofinite subset $z$ of $\omega$. We know that $\operatorname{tcl}(y) \in \mathbf{G}_{1}$ by the argument of the previous paragraph, the rank of $y$ not exceeding that of $x$, and that $\operatorname{tcl}(z)=\omega$, so that again $\operatorname{tcl}(x)$, being the union of a pair of elements of $\mathbf{G}_{1}$ is itself in $\mathbf{G}_{1}$.

Model 1: A model of ReS with full separation in which cartesian products are absent 
Consider, working in some suitable theory such as ZF, the class $\mathbf{A}_{1}$ of all transitive sets which contain but finitely many ordered pairs.

Then $\mathbf{M}_{1}=\bigcup \mathbf{A}_{1}$, which is the same as the class of all sets $x$ such that $\operatorname{tcl}(x)$ contains but finitely many ordered pairs, is supertransitive and contains all ordinals, and models Extensionality, AxPair, Sum Set, Infinity and full Separation, full foundation and TCo. $\omega \in \mathbf{M}_{1}$ but $\omega \times \omega$ is not. Indeed the cartesian product of an infinite set and a non-empty set is never there; but the cartesian product of two finite sets is there, so in this model a set $a$ is finite if and only if $a \times a \in V$.

4.2 REMARK Note also that the graph of addition is not present in this model, since its domain would be $\omega \times \omega$, and the domain can be recovered using the axioms of union and $\Delta_{0}$ separation.

4.3 REMARK $\mathcal{S}(\omega) \in \mathbf{M}_{1}$.

4.4 REMARK $\mathbf{M}_{1}$ contains no bijection between $\omega$ and $S(\omega)$. For a bijection would be an infinite set of ordered pairs. Indeed, $\mathbf{M}_{1}$ contains no functions with infinite domain!

\section{Model 1a}

Write $S(x)$ for the set of finite subsets of $x$. Then in $\mathbf{M}_{1}, S(\omega)$ exists, but $S(S(\omega))$ does not. Indeed if $a$ is infinite, $S(S(a))$ never exists. So let $\mathbf{M}_{1 a}$ be the set of members $x$ of $\mathbf{M}_{1}$ such that $S(y)$ exists in $\mathbf{M}_{1}$ for each member $y$ of $\operatorname{tcl}(\{x\}) . \mathbf{M}_{1 a}$ has the property that $a$ is finite iff $S(a)$ exists iff $\mathcal{P}(a)$ exists.

$\mathcal{S}(\omega) \notin \mathbf{M}_{1 a}$. What else is true in $\mathbf{M}_{1 a}$ ? Does it contain all ordinals ?

\section{Model 2: A model of ReS with full separation in which $[\omega]^{1}$ and} $[\omega]^{2}$ do not exist

Take $\mathbf{A}_{2}$ to be the class of those transitive $u$ such that $\{x \in u \mid \overline{\bar{x}} \leqslant 2\}$ is finite, and $\mathbf{M}_{2}$ to be $\bigcup \mathbf{A}_{2}$.

4.5 REMARK If we look at $\mathbf{C}$, the class of those $x$ such that $\operatorname{tcl}(x)$ contains only finitely many sets of cardinality 2 , we get a model that is nearly the same as the model $\mathbf{M}_{1}$; the chief difference seems to be that $[\omega]^{\omega}$ is not a member of $\mathbf{C}$, but is a member of $\mathbf{M}_{1}$.

4.6 REMARK We shall return to this mode of construction for Model 6.

\section{Model 3: ringing the changes}

Consider for any given $\mathfrak{k}$ the set $\mathbf{A}_{3, \mathfrak{k}}$ of those $u$ with $f_{u} O\left(n^{\mathfrak{k}}\right)$. This gives a model $\mathbf{M}_{3, \mathfrak{k}}$ of full separation in which Cartesian product will fail. $[\omega]^{\mathfrak{k}}$ will be in the model but not $[\omega]^{\mathfrak{k}+1}$. 
The arguments are modifications of those of [M1]: a similar argument is worked in detail below.

\section{Model 4: asymmetry of cartesian product}

Let $\mathbf{A}_{4}=\{u \mid u$ is transitive and $(V \times\{\omega\}) \cap u$ is finite $\}$.

Put $\mathbf{M}_{4}=\bigcup \mathbf{A}_{4}$. Then $\omega \times\{\omega\} \notin \mathbf{M}_{4}$, but both $\{\omega\} \times \omega$ and $\omega \times\{\omega+1\}$ are in $\mathbf{M}_{4}$.

4.7 In one of our later systems we would be able to define the right WienerKuratowski rank of a set by this rudimentary recursion:

$$
\varrho_{r W K}(x)= \begin{cases}0 & \text { if } x \text { is not an ordered pair } \\ 1+\varrho_{r W K}(\operatorname{right}(x)) & \text { otherwise }\end{cases}
$$

and prove that for any $x, \varrho_{r W K}(x)<\omega$.

For the moment we content ourselves with a weak form, for which $\mathrm{S}_{0}$ is adequate, and which will be useful for some of our model-building:

4.8 DEFINITION The weak right Wiener-Kuratowski rank is defined by cases:

$$
\varrho_{w r W K}(x)= \begin{cases}0 & \text { if } x \text { is not an ordered pair } \\ 1 & \text { if } x \text { is an ordered pair but right }(x) \text { is not } \\ 2 & \text { if both } x \text { and right }(x) \text { are ordered pairs }\end{cases}
$$

Now, for a variant of Model 4 , take $X$ to be the class of those sets of weak right WK rank 2 . Then $\omega \times(\omega \times \omega)$ will not be in $M_{X}$, whereas $(\omega \times \omega) \times \omega$ will be.

Hence we have the curiosity that in this model, there will be a bijection one way but not the other.

\section{MODELS OF DB}

\section{Model 5: A slim model for Devlin}

5.0 PROPOSITION There is a supertransitive model of DB containing all ordinals but omitting the set of finite sets of natural numbers.

Write $f_{u}$ for the map $n \mapsto \overline{\overline{u \cap V_{n}}}$. Write $g_{k}$ for the map $n \mapsto n^{k}$.

5.1 Definition Let $\mathbf{A}_{5}$ be the class of transitive sets $u$ such that the map $f_{u}$ is dominated (i.e. eventually majorised) by some $g_{k}$ Let $\mathbf{M}_{5}=\bigcup \mathbf{A}_{5}$. 
5.2 LEMMA $\mathbf{A}_{5} \subseteq \mathbf{M}_{5}$.

Proof: If $u \in \mathbf{A}_{5}$, then $u \in u \cup\{u\} \in \mathbf{A}_{5}$.

5.3 LEMMA $\mathbf{M}_{5}$ is transitive, being the union of transitive sets.

5.4 LEMMA $\mathbf{M}_{5}$ is supertransitive.

Proof : If $x \subseteq y \in u \in \mathbf{A}_{5}$ then $x \subseteq u$; put $v=u \cup\{x\} . v$ is transitive and for each $n \overline{\overline{v \cap V_{n}}} \leqslant \overline{\overline{u \cap V_{n}}}+1$, so $v \in \mathbf{A}_{5}$.

5.5 Corollary (Z) $\mathbf{M}_{5}$ models extensionality, difference, full foundation and full separation.

5.6 LEMMA $\omega \in \mathbf{M}_{5}$ : indeed, $\mathbf{A}_{5}$ contains all ordinals.

5.7 Lemma For each $k,[\omega]^{k}$ is in $\mathbf{M}_{5}$.

Proof : $u_{k}={ }_{\mathrm{df}} \omega \cup[\omega]^{k} \cup\left\{[\omega]^{k}\right\}$ is transitive. $\overline{\overline{\left(u_{k} \cap V_{n}\right)}}=\left(\begin{array}{l}n \\ k\end{array}\right)<n^{k}$.

5.8 REMARK Indeed for each $\kappa,[\kappa]^{k} \in \mathbf{M}_{5}$.

5.9 LEMMA $[\omega]^{<\omega}$ is not in $\mathbf{M}_{5}$,

Proof : Suppose $[\omega]^{\omega} \in u$, a transitive set. Then $\overline{\overline{u \cap V_{n}}} \geqslant 2^{n}$, and the map $n \mapsto 2^{n}$ eventually strictly dominates all the $n \mapsto n^{k}$ 's.

5.10 Corollary $\mathcal{P}(\omega) \notin \mathbf{M}_{5}$

5.11 LEMMA $\varnothing \in \mathbf{M}_{5}$.

5.12 LEMMA If $a$ and $b$ are in $\mathbf{M}_{5}$ so is $\{a, b\}$.

Proof : Let $a \in u \in \mathbf{A}_{5}$ and $b \in v \in \mathbf{A}_{5}$. Put $w=u \cup v$. Then $f_{w}$ is dominated by $f_{u}+f_{v}$, so if $f_{u}$ is dominated by $g_{k}$ and $f_{v}$ by $g_{\ell}$, then $f_{w}$ is dominated by $g_{\max (k, \ell)+1}$.

5.13 LEMma If $a$ is in $\mathbf{M}_{5}$, so is $\bigcup a$.

Proof : Let $a \in u \in \mathbf{A}_{5}$. Then $a \subseteq u$, so $\bigcup a \subseteq \bigcup u \subseteq u$; as before $\{\bigcup a\} \cup u$ will be in $\mathbf{A}_{5}$.

5.14 LEMma TCo holds in $\mathbf{M}_{5}$; indeed $x \in \mathbf{M}_{5} \Longrightarrow t c l(x) \in \mathbf{M}_{5}$.

Proof : Let $v=t \operatorname{cl}(x)$ where $x \in u \in \mathbf{A}_{5}$. Then $v \subseteq u$ and is therefore in $\mathbf{M}_{5}$ by supertransitivity.

5.15 LEMMA If $a$ and $b$ are in $\mathbf{M}_{5}$ so is $a \times b$.

Proof : it is enough to show that if $u$ is in $\mathbf{A}_{5}$, then $u^{\star} \in \mathbf{A}_{5}$. By the reasoning in the proof of Proposition 3.8, if $f_{u}$ is dominated by $g_{k}$ then $f_{u^{\star}}(n)$ for sufficiently large $n$ is at most $n^{k}+(n-1)^{k}+(n-1)^{2 k}+(n-2)^{2 k}$ 
which in turn is at most $4 g_{2 k}(n)$; thus $f_{u^{\star}}$ is dominated by $g_{2 k+1}$ and $u^{\star}$ is accordingly in $\mathbf{A}_{5}$.

5.16 Lemma if $x \in u \in \mathbf{A}_{5}$, then Dom $x \subseteq u$ and is thus in $\mathbf{M}_{5}$.

The following verifications are related to the finite axiomatisation of DB. We check that for $a$ in $\mathbf{M}_{5}$,

$$
\begin{gathered}
a \cap\left\{(p, q)_{2} \mid p \in q\right\} \in V \\
\{\langle q, p, r\rangle \mid\langle p, q, r\rangle \in a\} \in \mathbf{M}_{5} \\
\{\langle q, r, p\rangle \mid\langle p, q, r\rangle \in a\} \in \mathbf{M}_{5}
\end{gathered}
$$

The first is immediate by supertransitivity, and for the other two, if $a \in u \in \mathbf{A}_{5}$, both the given classes are contained in $u \times(u \times u)$, and are thus in $\mathbf{M}_{5}$ by supertransitivity.

5.17 REMARK The model being supertransitive, the set of even numbers is in it. That is of interest, because that was Gandy's test set, studied in Section 2. His arguments use quantifier elimination; our examples do not.

We show that $\mathbf{M}_{5}$ is not a model of GJ. Recall the definition of the Ackermann relation ACK $\subseteq \omega \times \omega: m$ ACK $n$ if and only if $2^{m}$ is one of the summands in the binary expression of $n$ as a sum of powers of 2 .

5.18 LEMMA ACK $\in \mathbf{M}_{5}$.

Proof : $\omega \times \omega \in \mathbf{M}_{5}$ and $\mathbf{M}_{5}$ is supertransitive.

5.19 Proposition $\mathbf{M}_{5}$ is not a model of $\mathrm{GJ}$.

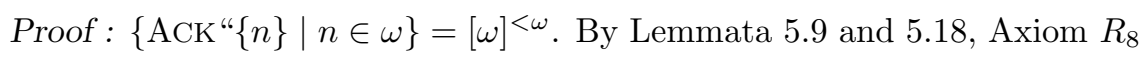
fails in $\mathbf{M}_{5}$.

5.20 REMARK The graph of addition is present in this model, as it will be in any supertransitive model of $\mathrm{DB}_{0}$ containing $\omega$; one may also argue directly that if $u$ is the transitive closure of the singleton of that graph, $f_{u}$ is dominated by $g_{3}$.

5.21 Remark Gandy's model $\mathbf{G}_{2}$, given below, is a model of GJ without the graph of addition; the submodel $\bigcup\left(\mathbf{G}_{2} \cap \mathbf{A}_{5}\right)$ will be supertransitive relative to $\mathbf{G}_{2}$, and will be a transitive model of DB in which GJ fails and in which the graph of addition is absent.

\section{Model 6}

We consider a variant of the construction $\mathbf{M}_{2}$ of section 2 .

Here we wish to study the extent to which DB proves the existence of the sets $[\omega]^{\mathfrak{k}}$ 
5.22 Proposition For any $\mathfrak{k} \geqslant 3$, DB, if consistent, fails to prove that $[\omega]^{\mathfrak{k}}$ exists.

Fix $\mathfrak{k} \geqslant 3$. We shall exhibit a supertransitive model $\mathbf{M}_{6, \mathfrak{k}}$ of $\mathrm{DB}$ in which $[\omega]^{\ell}$ exists iff $\ell \neq \mathfrak{k}$.

5.23 REMARK Indeed the existence of $[\omega]^{\ell}$ for different $\ell$ is independent. So we can code an arbitrary subset of $\omega$ into the theory of such a model.

Guided by Proposition 3.8, we let $X_{6, \mathfrak{k}}$ be the class of all sets of cardinality $\mathfrak{k}$, we take $\mathbf{A}_{6, \mathfrak{k}}$ to be the class of all transitive $u$ such that $u \cap X_{6, \mathfrak{k}}$ is finite, and $\mathbf{M}_{6, \mathfrak{e}}$ to be $\bigcup \mathbf{A}_{6, \mathfrak{e}}$. Then that will model $\mathrm{S}_{0}$ with full separation and full foundation; for $\mathfrak{k} \geqslant 3$, it will model Cartesian Product, since then for $u$ transitive, $X_{6, \mathfrak{k}} \cap\left([u]^{1} \cup[u]^{2} \cup(u \times u)\right)=\varnothing$, and so $u \in \mathbf{A}_{6, \mathfrak{k}} \Longrightarrow u^{\star} \in \mathbf{A}_{6, \mathfrak{k}}$.

If $\mathfrak{l} \neq \mathfrak{k}$, then for each $x$ in $\mathbf{M}_{6, \mathfrak{k}},[x]^{\mathfrak{l}}$ will be in $\mathbf{M}_{6, \mathfrak{k}}$ : if $x \in u \in \mathbf{A}_{6, \mathfrak{k}}$, $[x]^{\mathfrak{l}} \subseteq[u]^{\mathrm{l}} ; u \cup[u]^{\mathrm{l}}$ is transitive, and its intersection with $X_{6, \mathfrak{k}}$ equals $u \cap X_{6, \mathfrak{k}}$, and is therefore finite. By the supertransitivity of $\mathbf{M}_{6, \mathfrak{k}},[x]^{\mathfrak{l}} \in \mathbf{M}_{6, \mathfrak{k}}$.

On the other hand for no infinite member $x$ of $\mathbf{M}_{6, \mathfrak{k}}$ will $[x]^{\mathfrak{k}}$ be in $\mathbf{M}_{6, \mathfrak{k}}$, as no member of $\mathbf{M}_{6, \mathfrak{k}}$ can have infinitely many members of cardinality $\mathfrak{k}$.

So it will also be true that ${ }^{\mathfrak{k}} \omega$ is not in the model, although $\omega \times(\omega \times(\ldots))$ (k times) will be.

5.24 REMARK Consider the case $\mathfrak{k}=3$ : the graph of addition, implemented (as we do) as a subset of $\omega \times(\omega \times \omega)$, is a member of $\mathbf{M}_{6,3}$, but implemented as a set of 3 -tuples is not, since in that model, no infinite subset of ${ }^{3} \omega$ exists. Thus these weak theories are extremely sensitive to the implementation of functions, a point that is touched on by Stanley in his review [St] of Devlin's book [De].

5.25 REMARK If we ask that for each $k u$ contains only finitely many sets of size $k$, the resulting model, though containing all the ordinals, will contain none of the sets $[\omega]^{k}$; if we ask for $u$ to contain only finitely many finite sets, the resulting model will be $\mathbf{H F}$, given that we are using the Axiom of Foundation. In a universe with Quine atoms, of course, the situation would be different.

\section{A variant of Model 6}

Let $\mathbf{A}=\left\{u \mid \bigcup u \subseteq u \& u \cap{ }^{3}[\omega, \omega+\omega)\right.$ is finite $\}$, and let $\mathbf{M}=\bigcup \mathbf{A}$. Then $\mathbf{H F} \in \mathbf{M}$ but ${ }^{3}[\omega, \omega+\omega)$ is not. $\mathbf{M}$ contains all ordinals and is a supertransitive model of BS.

\section{Model 7: a failure of $\bigcup$ "}

Here we shall exhibit a transitive model of BS in which the following failure of GJ occurs: there is a set $B$ such that $\{\bigcup x \mid x \in B\}$ is not a set. 
Following Remark 3.9, take $X$ to be the class of transitive sets of limit rank, $\mathbf{A}_{7}$ to be $A_{X}$, the class of all transitive sets $u$ such that only finitely many transitive sets of limit rank are members of $u$, and $\mathbf{M}_{7}$ to be $\bigcup \mathbf{A}_{7}$.

Then $\mathbf{M}_{7}$ is a supertransitive model of $\mathrm{ReS}_{0}+$ full foundation $+\mathrm{TCo}$; " $x \times y \in V$ " will be true in it since for $u$ transitive, $u^{\star} \cap X=u \cap x$, as all members of $[u]^{1} \cup[u]^{2} \cup(u \times u)$ are non-empty finite sets and therefore of successor rank; and it contains all the ordinals below $\omega^{2}$, and thus models the axiom of infinity. To prove the failure of GJ, we turn to the idea of a Zermelo tower from [M1], which is defined thus:

5.26 DeFinition For $a$ any set, put

$Z_{0}(a)=\varnothing ; Z_{1}(a)=\{a\} ; Z_{n+1}(a)=\{a\} \cup\left(\mathcal{P}\left(Z_{n}(a)\right) \backslash\{\varnothing\}\right) ; Z(a)=$ $\bigcup_{n \in \omega} Z_{n}(a)$.

If one thinks of $\mathbf{H F}$ as a collection of words in $\varnothing,\{$ and $\}$ then $Z(a)$ is the collection of the corresponding words with $a$ substituted for $\varnothing$ throughout. Thus every member either is a finite non-empty set or equals $a$.

Now let $\mathcal{X}$ be the set of those subsets $a$ of $\omega+1$ of which $\omega$ is a member.

For each such $a$ let $x(a)={ }_{\mathrm{df}}\left\{Z_{n}(a) \mid n \in \omega\right\}$. The rank of $x(a)$ is $\omega+\omega$.

Let $x^{*}(a)=x_{a} \cup\{\omega+1\}$. All the members of $x^{*}(a)$ are of successor rank, and so $x^{*}(a)$ is not transitive, but $\bigcup x^{*}(a)=Z(a) \cup(\omega+1)$ which is transitive, and of rank $\omega+\omega$; its only transitive member of limit rank is $\omega$; thus each $x^{*}(a)$ is in $\mathbf{M}_{7}$.

Take $B$ to be $\left\{x^{*}(a) \mid a \in \mathcal{X}\right\}$. Note that

$$
\operatorname{tcl}(\{B\})=\{B\} \cup B \cup\left\{Z_{n}(a) \mid n \in \omega \& a \in \mathcal{X}\right\} \cup\{\omega+1\} \cup \omega+1,
$$

a transitive set of which the sole transitive member of limit rank is $\omega$. Hence $B \in \mathbf{M}_{7}$; but $\{\bigcup x \mid x \in B\}$ will not be, since it is an infinite set of transitive sets of limit rank.

\section{Model 8: in which $\mathcal{S}(\omega)$ exists but not $\mathcal{S}(\omega \times \omega)$}

Note that the cardinality of $\mathcal{S}(\omega \times \omega) \cap V_{n}$ is about $2^{(n-2)^{2}}$, an order of magnitude higher than that of $\mathcal{S}(\omega) \cap V_{n}$; we have to take the transitive closure of course, but that will only make it higher.

So take $\mathbf{A}_{8}$ to be the class of all transitive $u$ such that the map $f_{u}$ defined by $f_{u}(n)=\overline{\overline{u \cap V_{n}}}$ is eventually dominated, for some $k$, by $n \mapsto 2^{k n}$, and $\mathbf{M}_{8}$ to be $\bigcup \mathbf{A}_{8}$.

By Proposition 3.4 and Remark 3.8, $\mathbf{M}_{8}$ models BS.

5.27 REMARK By estimating the number of ordered triples in $V_{n}$, and considering those transitive $u$ with $f_{u}$ dominated by $n \mapsto 2^{k n^{2}}$ for some $k$, we would obtain a model containing $\mathcal{S}(\omega \times \omega)$ but omitting $\mathcal{S}(\omega \times(\omega \times \omega))$. 


\section{Model 9: a failure of Seq}

To get a model of BS plus $\mathbf{H F} \in V+$ a failure of Seq: let $\mathbf{A}_{9}$ be $\left\{u \mid \bigcup u \subseteq u \& u \cap{ }^{3} A\right.$ is finite $\}$, where we have yet to choose $A$.

5.28 LEMMA $\mathbf{H F} \cap{ }^{3} A={ }^{3}(\mathbf{H F} \cap A)$.

So take $A$ to be $\{\omega\} \times \omega$. The resulting model $\mathbf{M}_{9}=\bigcup \mathbf{A}_{9}$ will have HF as a member; ${ }^{3}(\{\omega\} \times \omega)$ will not be there, but ${ }^{3}(\omega \times\{\omega\})$ will be.

The model will contain a bijection between the two sets $\omega \times\{\omega\}$ and $\{\omega\} \times \omega$, and therefore will fail to model GJ.

We should check that $\star$-closure holds in Model 9. Recall that $u^{\star}=$ $u \cup[u]^{1} \cup[u]^{2} \cup u \times u$.

The members of ${ }^{3} A$ are 3 -sequences, which are neither singletons nor doubletons nor ordered pairs. So in this case

$$
u^{\star} \cap{ }^{3} A=u \cap{ }^{3} A,
$$

and all is well.

5.29 REMARK In the next section we give Gandy's model of GJ, thus containing each $\operatorname{Seq}(u, a, n)$ but not, for $a=\omega$, the set of all finite sequences from $a$.

\section{Model 10: from sheer perversity}

Let $P$ be an almost disjoint family of infinite subsets of $\omega$; for $X$ in $P$, consider the class $A_{X}$ of all transitive sets having finite intersection with ${ }^{3} X$. Take for $Q$ any subset of $P, \mathbf{A}_{Q}$ to be the intersection of all the $A_{X}$ for $X \in Q$. Then, for $X$ in $P, \cup \mathbf{A}_{Q}$ will contain ${ }^{3} X$ iff $X$ is not in $Q$, and will model BS.

\section{MODELS OF GJ}

\section{Gandy: A set that models GJ but not fReR}

Take $\mathbf{G}_{2}$ to be the rudimentary closure of $\{\omega\}$.

The set of even numbers is not in $\mathbf{G}_{2}$, not being $\Delta_{0} . \Pi_{1}$, indeed full, foundation is true in $\mathbf{G}_{2}$; TCo will be true there as $\omega$ is transitive, by Proposition 2.83. But as we saw in $\S 2$, fReR proves the existence of EVEN.

The next two remarks are semantical versions of [G, Theorems 2.2.2(ii) and 3.1.1].

6.0 REMARK It follows that the graph $G$ of addition is not a member of this model, for

$$
\operatorname{EVEN}=\omega \cap\{n \mid n=0 \vee \exists m: \in n(n, m, m) \in G\} .
$$


6.1 REMARK The graph of concatenation is not in this model.

The unprovability of $\mathcal{S}(\omega) \in V$ in GJ

6.2 REMARK If $\Delta_{0}$ separation is true and $S(\omega) \in V$, then the set of even numbers can be built as

$$
\bigcup(S(\omega) \cap\{x \mid x \subseteq \omega \& 0 \in x \& \forall n:<\bigcup x(n \in x \Longleftrightarrow n+1 \notin x)\})
$$

6.3 Corollary " $S(\omega) \in V$ " is false in the rud closure of $\{\omega\}$.

Proof : by Gandy, who showed that EvEN is not there.

6.4 Corollary " $S(\omega) \in V$ " is not provable in GJ.

6.5 Corollary Since the existence of $\mathcal{S}(\omega)$ is derivable in GJ from the existence of ACK, the existence of ACK is not provable in GJ.

\section{MODELS OF fReR AND BEYOND}

\section{Gandy: A set that models fReR but not ReR}

Take $\mathbf{G}_{3}$ to be $V_{\omega+\omega}$.

\section{Model 11:}

Write $\mathbf{H C}$ for the union of all countable transitive sets. Then $\mathbf{M}_{11}=_{\mathrm{df}}$ $V_{\omega+\omega} \cap \mathbf{H C}$, that is, the union of all countable transitive sets of rank less than $\omega+\omega$, is a model of fReRI but not, by Proposition 2.102, ReR. Assuming choice for countable families,

\section{Variants of Model 11:}

As often in this paper, we can obtain further models by carrying out one construction within another. Let $\mathbf{N}$ be an admissible set of height $\kappa>\omega$. For $0<\eta=\bigcup \eta<\kappa$, Let $\mathbf{N}_{11, \eta}$ be the union of transitive sets in $M$ of rank less than $\eta$. Then that will be a model of $\mathrm{fReC}$, and of AxInf if $\eta>\omega$. For a second example, assume that $\mathrm{AC}$ holds in $\mathbf{N}$ and consider the union $\mathbf{P}$ of all transitive sets which are members of $\mathbf{N}$ and countable there. Then $\mathbf{P}$ will be a model of fReC. Further $\mathbf{P}$ will be a model of $\mathcal{S}(x) \in V$.

\section{Model 12: of fReR omitting HF}

Since fRe $R_{0}$ is a subtheory of $Z$, it is enough to find a transitive model of $Z$ in which $\mathbf{H F}$ is not a set. The construction of one such model is sketched in Remark 14.24; for others, see [M1] and the further references there. 
7.0 Problem For which $\lambda$ and $\alpha$ are $L_{\lambda}$ and $J_{\alpha}$ a model of fReR or fReC? Material in a later section suggests that a necessary condition will be that $\alpha=\omega \alpha$. Is that also sufficient?

Zarach: a set that models ReR but not KPI

See $[\mathrm{Z}]$, Theorem 6.4.

Model 13: a model of $Z+$ TCo in which rank is not everywhere defined

Let $\lambda$ be a limit ordinal. Define

$$
\mathbf{A}_{13, \lambda}={ }_{\mathrm{df}}\{u \mid \bigcup u \subseteq u \& u \cap \lambda<\lambda\} ; \quad \mathbf{M}_{13, \lambda}=\bigcup \mathbf{A}_{13, \lambda} ;
$$

Note that if $u$ and $v$ are members of $\mathbf{A}_{13, \lambda}$ then $u \cup v \in \mathbf{A}_{13, \lambda}$, and that $u \cup \mathcal{P}(u) \cup\{\mathcal{P}(u)\}$; so $\mathbf{M}_{13, \lambda}$ will be a supertransitive model of all of $\mathbf{Z}$ except (in the case $\lambda=\omega$ ) the axiom of infinity. As $\mathbf{A}_{13, \lambda} \subseteq \mathbf{M}_{13, \lambda}, M$ will also model TCo. $V_{\lambda}$ will be a subclass but not a member of $\mathbf{M}_{13, \lambda}$; $O N \cap \mathbf{M}_{13, \lambda}=\lambda$. $V_{\lambda}$ will be definable in $\mathbf{M}_{13, \lambda}$ as the class of those sets which lie in the domain of an attempt at the rank function. The union of those attempts will be a class but not a set of $\mathbf{M}_{13, \lambda}$.

We show that $\mathbf{M}_{13, \lambda}$ will contain sets of all ranks. Let $u$ be any member of $\mathbf{A}_{13, \lambda}$ which is not an ordinal. Define the sequence

$$
u_{0}=u ; u_{\nu+1}=u_{\nu} \cup\left\{u_{\nu}\right\} ; u_{\eta}=\bigcup_{\nu<\eta} u_{\nu} \quad \text { for } 0<\eta=\bigcup \eta .
$$

Then it is easily shown by induction on $\nu$ that no $u_{\nu}$ is an ordinal; that each $u_{\nu}$ is transitive; that each $u_{\nu}$ is a member of each $u_{\nu^{\prime}}$ with $\nu<\nu^{\prime}$; that $\varrho\left(u_{\nu}\right)=\varrho\left(u_{0}\right)+\nu$; that $u_{\nu} \cap O N=u_{0} \cap O N$; and hence that each $u_{\nu}$ is in $\mathbf{A}_{13, \lambda}$ and therefore in $\mathbf{M}_{13, \lambda}$.

The case $\lambda=\omega$ gives us a model of $\mathbf{Z}$ which has infinite members but for which the axiom of infinity in the form $\omega \in V$ is false.

\section{ADDING $\mathcal{S}(x) \in V$ TO THESE SYSTEMS}

Devlin in his book [Dev] had the aim of finding a theory that would hold in all structures $L_{\lambda}$ for $\lambda$ a limit ordinal, and in all structures $J_{\alpha}$ for $\alpha$ an arbitrary non-zero ordinal, be strong enough for a unified development of both hierarchies, and yet not require the introduction of rudimentary functions at too early a stage; and proposed BS as such a theory. Alas, it is not, as we shall see in the next section through the use of the models that 
we have built in earlier sections. Devlin's treatment is further flawed by other mistakes such as those mentioned by Stanley in his review (Journal of Symbolic Logic 53 pp 864-8) of Devlin's book Constructibility, where Solovay (unpublished) is quoted as declaring [Dev, I.9.5] to be false "as can be seen by a forcing argument," and [Dev I.9.3] to be refutable "by the use of Ehrenfeucht games."

Stanley concludes his review of [Dev] by asking whether such a theory might be found. We have a candidate: our proposal is to add to the axioms of DB the axioms

$$
\omega \in V \text { and } \mathcal{S}(x) \in V
$$

where $\mathcal{S}(x)$ is to mean the set of finite subsets of $x$. Call the resulting system DS, for "Devlin strengthened", and call ReSS, GJS, fReRS the result of adding to ReS, GJ, and fReR the same two principles. Note that whereas BS had full foundation, we allow DS and our other systems to have only $\Pi_{1}$ foundation.

8.0 Proposition The existence of Cartesian products is provable in ReSS: so DS is the same as ReSS.

Proof : given $a, \mathcal{S}(a)$ will contain all 1- and 2-element subsets of $a$; hence $a \times a$ is a $\Delta_{0}$ subclass of the set $\mathcal{S}(\mathcal{S}(a))$; to form $b \times c$, take $a=b \cup c$ and apply $\Delta_{0}$ Separation.

At the stronger end of our lattice of theories, the enhancement amounts to no more than adding the axiom of infinity, since by Proposition 2.103, ReRI proves that $\forall x \mathcal{S}(x) \in V$.

8.1 PRoBlem Is TCo derivable from the other axioms of ReR ?

8.2 REMARK It is tempting to add a further axiom,

$$
\mathbf{H F} \in V,
$$

which in many ways makes life easier, because $\mathbf{H F}$ is a model of ZF Infinity, and therefore a large number of functions become automatically available. But a feeling, that doing so does not address the chief problem with BS, is reinforced by the variant given above of Model 6 , in which $\mathbf{H F}$ exists but some ${ }^{3} x$ not.

Our aim in this section is to study these systems, and we shall begin by enlarging our syntax to treat a class of formulæ that is slightly more general than $\Delta_{0}$ but still limited in a specific sense.

\section{A syntactical enhancement}


We examine the consequences of allowing limited quantifiers $\forall y: \in \mathcal{S}(x)$, $\exists y: \in \mathcal{S}(x)$. The paradigm for our discussion is section 6 of "The Strength of Mac Lane Set Theory" where the quantifiers $\forall y: \in \mathcal{P}(x)$, written as $\forall y: \subseteq x$ were discussed.

We call a formula $\Delta_{0, \mathcal{S}}$ if all its quantifiers are of the form $Q x: \in \mathcal{S}(y)$ or $Q x: \in y$ where $Q$ is $\forall$ or $\exists$, and $x$ and $y$ are distinct variables. We preserve "restricted" as a description of the quantifiers $Q x: \in y$, and speak of the occurrences of $y$ in $Q x: \in \mathcal{S}(y)$ or $Q x: \in y$ as limiting the range of the bound variable $x$.

It is tempting, indeed, to adopt a different presentation of the language by declaring the class of atomic formulæ to consist of every formula of one of the three forms

$$
x \in y \quad x=y \quad x \in \mathcal{S}(y)
$$

and to have three kinds of quantifiers, $\forall x, \forall x: \in y$ and $\forall x: \in \mathcal{S}(y)$ in the language; but we shall not formally adopt this approach here. Gandy in his paper $[\mathrm{G}]$ suggests considering the ancestral $\in^{*}$ of $\in$, where $x \in^{*} y$ iff $x \in \operatorname{tcl}(y)$, which will become easily available in our system.

8.3 Proposition (DS) " $x \in \mathcal{S}(y) ", " x=\mathcal{S}(y)$ " and " $\mathcal{S}(y) \in x$ " are all $\Delta_{0, \mathcal{S}}$.

\section{Normal forms for $\Delta_{0, \mathcal{S}}$ formulæ}

8.4 We sketch a method of rewriting a $\Delta_{0, \mathcal{S}}$ formula so that all variables are limited by terms constructed from the free variables of the original formula using only $\bigcup$; thus ultimately the terms limiting variables contain no variables that are themselves bound by other quantifiers.

Unlike $\in, \subseteq$ is transitive. Hence the following reduction is available:

$$
\exists x: \in \mathcal{S}(t) \forall y: \in \mathcal{S}(x) \mathfrak{A} \Longleftrightarrow \exists x: \in \mathcal{S}(t) \forall y: \in \mathcal{S}(t)[y \subseteq x \Longrightarrow \mathfrak{A}]
$$

Note here that on the left hand side the $x$ limiting $y$ in the quantifier $\forall y: \in \mathcal{S}(x)$ is itself bound by the preceding quantifier $\exists x: \in \mathcal{S}(t)$, whereas on the right hand side the $t$ that limits both quantifiers is itself free. We may speak of $t$ in the above displayed formula or $\bigcup t$ in the next as a free term.

We thus obtain these reductions: 


$$
\begin{aligned}
\forall x: \in a \exists y: \in x \mathfrak{A} & \Longleftrightarrow \forall x: \in a \exists y: \in \bigcup a[y \in x \& \mathfrak{A}] ; \\
\forall x: \in \mathcal{S}(a) \exists y: \in x \mathfrak{A} & \Longleftrightarrow \forall x: \in \mathcal{S}(a) \exists y: \in a[y \in x \& \mathfrak{A}] ; \\
\forall x: \in a \exists y: \in \mathcal{S}(x) \mathfrak{A} & \Longleftrightarrow \forall x: \in a \exists y: \in \mathcal{S}(\bigcup a)[y \subseteq x \& \mathfrak{A}] \\
& \Longleftrightarrow \forall x: \in a \exists y: \in \mathcal{S}(\bigcup a) \\
& {\left[\forall s_{1}: \in \bigcup a\left(s_{1} \in y \Longrightarrow y_{1} \in x\right) \& \mathfrak{A}\right] ; } \\
\forall x: \in \mathcal{S}(a) \exists y: \in \mathcal{S}(x) \mathfrak{A} & \Longleftrightarrow \forall x: \in \mathcal{S}(a) \exists y: \in \mathcal{S}(a)[y \subseteq x \& \mathfrak{A}] \\
& \Longleftrightarrow \forall x: \in \mathcal{S}(a) \exists y: \in \mathcal{S}(a) \\
& {\left[\forall s_{2}: \in a\left(s_{2} \in y \Longrightarrow s_{2} \in x\right) \& \mathfrak{A}\right] . }
\end{aligned}
$$

Those equivalences, which are all valid in $\mathrm{S}_{0}$, and, where applicable, preserve the stratifiability of the formula under consideration, show that one may progressively rewrite the formula to one in which all limitations are of the form $: \in \mathcal{S}\left(\bigcup^{\mathfrak{k}} a\right)$ or $: \in \bigcup^{\mathfrak{k}} a$ with $a$ a free variable. We call such a formula one in free form. Our expansion of $y \subseteq x$ in the fourth and sixth lines, which would be unnecessary if we treated $y \subseteq x$ as atomic, helps to secure free form. We call the bound variables $s_{i}$ introduced in those expansions subsidiary variables: we shall suppress mention of them in our discussion below, so that when we speak of "every quantifier", we mean "every quantifier binding other than a subsidiary variable".

Given a formula in free form, we replace each limiting free term by a new variable and add a clause expressing the equality of the term and the variable.

We have reached the

8.5 First Limited Normal Form Let $\Phi$ be a $\Delta_{0, \mathcal{S}}$ formula with free variables $a_{0}, \ldots a_{\mathfrak{n}}$. Let $\mathfrak{m}+1$ be the number of quantifiers occurring in $\Phi$. Then for $0 \leqslant j \leqslant \mathfrak{m}$, there are numbers $0 \leqslant \mathfrak{k}(j) \leqslant \mathfrak{n}, 0 \leqslant \mathfrak{l}(j)$, determined by the quantifier structure of $\Phi$, new variables $y_{0}, \ldots y_{\mathfrak{m}}$, and a $\Delta_{0, \mathcal{S}}$ formula $\Psi_{1}$ with free variables $a_{0}, \ldots a_{\mathfrak{n}}, y_{0}, \ldots y_{\mathfrak{m}}$, in which every quantifier is limited by one of the parameters $y_{i}$, such that, abbreviating $\forall y_{0}, \ldots, \forall y_{\mathfrak{m}}$ by $\forall \vec{y}$, we have

$$
\vdash_{\mathrm{DB}_{0}} \overrightarrow{\forall a} \overrightarrow{\forall y}\left[\bigwedge_{0 \leqslant j \leqslant \mathfrak{m}} y_{j}=\bigcup^{\mathrm{r}(j)} a_{\mathfrak{k}(j)} \Longrightarrow\left[\Phi(\vec{a}) \Longleftrightarrow \Psi_{1}(\vec{a}, \vec{y})\right]\right]
$$

To take things to a second stage, if we know that we intend using the formula $\Phi(a)$ in a context where $a_{i}$ will be constrained to be a member of 
$b_{i}$, we may replace the restriction $: \in \bigcup^{\mathfrak{l}} a_{i}$ by the restriction $: \in \bigcup^{\mathfrak{l}+1} b_{i}$; and each limitation $: \in \mathcal{S}\left(\bigcup^{\mathfrak{l}} a_{i}\right)$ by the limitation $: \in \mathcal{S}\left(\bigcup^{\mathfrak{l}+1} b_{i}\right)$, since if $a \in b$, $\bigcup^{\mathrm{l}} a \subseteq \bigcup^{\mathfrak{l}+1} b$, and make a corresponding adjustment to the matrix.

We could also consider intended limitations $a_{i} \subseteq b_{i}$ instead of restrictions $a_{i} \in b_{i}$ : the replacements to be made then would be $: \in \bigcup^{\mathrm{l}} a_{i}$ by $: \in \bigcup^{\mathfrak{l}} b_{i}$ and $: \in \mathcal{S}\left(\bigcup^{\mathfrak{l}} a_{i}\right)$ by $: \in \mathcal{S}\left(\bigcup^{\mathfrak{l}} b_{i}\right)$, since if $a \subseteq b$ then $\bigcup^{\mathfrak{l} a} \subseteq \subseteq \bigcup^{\mathfrak{l}} b$.

Further, we could mix our intentions, and also leave some $a_{i}$ untouched, which is tantamount to saying $a_{i}=b_{i}$. We thus have the

8.6 SeCond Limited Normal Form Continuing the notation of the First Limited Normal Form, let $R, S$ and $U$ be disjoint sets partitioning $[0, \mathfrak{n}]$, and let $b_{0}, \ldots, b_{\mathfrak{n}}$ be variables not occurring in $\Phi$. Then for the same numbers $\mathfrak{k}(j), \mathfrak{l}(j)$, there is a $\Delta_{0, \mathcal{S}}$ formula $\Psi_{2}$ with free variables $a_{0}, \ldots a_{\mathfrak{n}}, y_{0}, \ldots y_{\mathfrak{m}}$, in which every quantifier is limited to one of the parameters $y_{i}$, such that

$$
\begin{aligned}
& \vdash_{\mathrm{DB}_{0}} \overrightarrow{\forall b} \overrightarrow{\forall a} \forall \overrightarrow{\forall y}\left[\left[\bigwedge_{i \text { in } R} a_{i} \in b_{i} \& \bigwedge_{i \text { in } S} a_{i} \subseteq b_{i} \& \bigwedge_{i \text { in } U} a_{i}=b_{i} \&\right.\right. \\
& \qquad \bigwedge_{\mathfrak{k}(j) \text { in } R} y_{j}=\bigcup^{\mathrm{I}(j)+1} b_{\mathfrak{k}(j)} \& \bigwedge_{\substack{\mathfrak{k}(j) \text { in } \\
S \text { or } U}} y_{j}\left.=\bigcup^{\mathrm{r}(j)} b_{\mathfrak{k}(j)}\right] \Longrightarrow \\
&\left.\Longrightarrow\left[\Phi(\vec{a}) \Longleftrightarrow \Psi_{2}(\vec{a}, \vec{y})\right]\right]
\end{aligned}
$$

8.7 EXAMPLE Let $\mathfrak{A}$ be quantifier-free, with six variables $a, b, x, y, z, w$. Suppose we want to re-write the formula

$$
\exists x: \in a \forall y: \in \mathcal{S}(x) \exists z: \in x \forall w: \in \mathcal{S}(z) \mathfrak{A}(a, b, x, y, z, w) .
$$

Let $\mathfrak{B}(a, b, x, y, z, w)$ be the formula

$$
(y \subseteq x \Longrightarrow[z \in x \&(w \subseteq z \Longrightarrow \mathfrak{A}(a, b, x, y, z, w))]) .
$$

Notice that $\mathfrak{B}$ is $\Delta_{0}$, or indeed quantifier-free if we count $s \subseteq t$ as atomic. Then

$$
\begin{aligned}
\exists x & : \in a \forall y: \in \mathcal{S}(x) \exists z: \in x \forall w: \in \mathcal{S}(z) \mathfrak{A}(a, b, x, y, z, w) \Longleftrightarrow \\
& \Longleftrightarrow \exists x: \in a \forall y: \in \mathcal{S}(\bigcup a) \exists z: \in \bigcup a \forall w: \in \mathcal{S}(\bigcup \bigcup a)[\mathfrak{B}(a, b, x, y, z, w)] .
\end{aligned}
$$


In order not to use $\mathcal{S}$ applied to a term that is not a variable, we introduce further variables $z_{j}$.

8.8 First ReStricted NORMAL Form Continuing the notation of the First Limited Normal Form, for the same numbers $\mathfrak{k}(j), \mathfrak{l}(j)$, there is a partition of $\{j \mid 0 \leqslant j \leqslant \mathfrak{m}\}$ into disjoint sets $L_{\Phi}, R_{\Phi}$; there are new variables $y_{j}, z_{j}$ for $0 \leqslant j \leqslant \mathfrak{m}$; and there is a $\Delta_{0}$ formula $\Psi_{3}$, with free variables the $a$ 's and the $z$ 's; such that every quantifier in $\Psi_{3}$ is restricted to one of the parameters $z_{i}$, and

$$
\begin{aligned}
& \vdash_{\mathrm{DB}_{0}} \overrightarrow{\forall a} \overrightarrow{\forall y} \forall \overrightarrow{\forall z}\left[\bigwedge_{j \text { in } R_{\Phi}}\left(z_{j}=y_{j} \& y_{j}=\bigcup^{\mathfrak{l}(j)} a_{\mathfrak{k}(j)}\right) \&\right. \\
& \left.\& \bigwedge\left(z_{j}=\mathcal{S}\left(y_{j}\right) \& y_{j}=\bigcup^{\mathfrak{r}(j)} a_{\mathfrak{k}(j)}\right)\right] \Rightarrow \\
& j \text { in } L_{\Phi} \\
& \left.\Rightarrow\left[\Phi(\vec{a}) \Longleftrightarrow \Psi_{3}(\vec{a}, \vec{z})\right]\right]
\end{aligned}
$$

Taking that to the corresponding second stage, and noting that if $a \subseteq b$ then $\mathcal{S}\left(\bigcup^{\mathfrak{l}} a\right) \subseteq \mathcal{S}\left(\bigcup^{\mathfrak{l}} b\right)$, whereas if $a \in b, \mathcal{S}\left(\cup^{\mathfrak{l}} a\right) \subseteq \mathcal{S}\left(\bigcup^{\mathfrak{l}+1} b\right)$, we reach the

8.9 SeCOnd ReStricted Normal Form Let $\Phi$ be a $\Delta_{0, \mathcal{S}}$ formula with free variables $a_{0}, \ldots a_{\mathfrak{n}}$. Let $R, S$ and $U$ be disjoint sets partitioning $[0, \mathfrak{n}]$, and let $b_{0}, \ldots, b_{\mathfrak{n}}$ be variables not occurring in $\Phi$. Let $\mathfrak{m}+1$ be the number of quantifiers occurring in $\Phi$. Then there is a partition of $\{j \mid 0 \leqslant j \leqslant \mathfrak{m}\}$ into disjoint sets $L_{\Phi}, R_{\Phi}$; for $0 \leqslant j \leqslant \mathfrak{m}$, there are numbers $0 \leqslant \mathfrak{k}(j) \leqslant \mathfrak{n}$, $0 \leqslant \mathfrak{l}(j)$, determined by the quantifier structure of $\Phi$, there are new variables $y_{j}, z_{j}$ for $0 \leqslant j \leqslant \mathfrak{m}$; and there is a $\Delta_{0}$ formula $\Psi_{4}$ with free variables the $a$ 's 
and the $z$ 's, in which every quantifier is restricted to one of the parameters $z_{i}$; such that,

$$
\begin{aligned}
& \vdash_{\mathrm{DB}_{0}} \overrightarrow{\forall b} \overrightarrow{\forall a} \forall \overrightarrow{y y} \overrightarrow{\forall z}\left[\left[\bigwedge_{i \text { in } R} a_{i} \in b_{i} \& \bigwedge_{i \text { in } S} a_{i} \subseteq b_{i} \& \bigwedge_{i \text { in } U} a_{i}=b_{i} \quad \&\right.\right. \\
& \& \bigwedge\left(z_{j}=y_{j} \& y_{j}=\bigcup^{\mathfrak{l}(j)+1} b_{\mathfrak{k}(j)}\right) \& \\
& \begin{array}{l}
j \text { in } R_{\Phi}, \\
\mathfrak{k}(j) \text { in } R
\end{array} \\
& \text { \& } \bigwedge\left(z_{j}=\mathcal{S}\left(y_{j}\right) \& y_{j}=\bigcup^{\mathfrak{l}(j)+1} b_{\mathfrak{k}(j)}\right) \& \\
& \begin{array}{l}
j \text { in } L_{\Phi}, \\
\mathfrak{k}(j) \text { in } R
\end{array} \\
& \text { \& } \bigwedge\left(z_{j}=y_{j} \& y_{j}=\bigcup^{\mathfrak{l}(j)} b_{\mathfrak{k}(j)}\right) \& \\
& j \text { in } R_{\Phi} \text {, } \\
& \mathfrak{k}(j) \text { in } S \text { or } U \\
& \left.\& \bigwedge_{j \text { in } L_{\Phi},}\left(z_{j}=\mathcal{S}\left(y_{j}\right) \& y_{j}=\bigcup^{\mathfrak{r}(j)} b_{\mathfrak{k}(j)}\right)\right] \Longrightarrow \\
& \mathfrak{k}(j) \text { in } S \text { or } U \\
& \left.\Longrightarrow\left[\Phi(\vec{a}) \Longleftrightarrow \Psi_{4}(\vec{a}, \vec{z})\right]\right]
\end{aligned}
$$

\section{Self-strengthening of DS}

We may now deduce the

8.10 METATHEOREM DS proves all instances of the scheme of $\Delta_{0, \mathcal{S}}$ separation.

Proof: Suppose that there are $\mathfrak{m}+1$ quantifiers in the $\Delta_{0, \mathcal{S}}$ formula $\Phi(x, a)$. By the Second Restricted Normal Form, we know that there are new variables $y_{0}, \ldots, y_{\mathfrak{m}}, z_{0}, \ldots, z_{\mathfrak{m}}$ and a $\Delta_{0}$ formula $\Psi_{4}(x, \vec{a}, \vec{z})$ with the free variables shown, such that

$\mathrm{DB}_{0} \vdash x \in d \&\langle$ conditions on $\vec{z}, \vec{y}, d$ and $\vec{a}\rangle \Longrightarrow\left[\Phi(x, \vec{a}) \Longleftrightarrow \Psi_{4}(x, \vec{a}, \vec{z})\right]$,

where there are $\mathfrak{m}+1$ conditions, each of one of the four following types, according to the quantifier structure of $\Phi$ :

$$
\begin{gathered}
{\left[z=y \& y=\bigcup^{\mathfrak{l}+1} d\right] ;\left[z=\mathcal{S}(y) \& y=\bigcup^{\mathfrak{l}+1} d\right]} \\
{\left[z=y \& y=\bigcup^{\mathfrak{l}} a\right] ;\left[z=\mathcal{S}(y) \& y=\bigcup^{\mathfrak{l} a}\right] .}
\end{gathered}
$$


In DS we may prove that given $d$ and $\vec{a}$ there are $y$ 's and $z$ 's satisfying the conditions, and for those $z$, we have $\forall x: \in d\left(\Phi(x, \vec{a}) \Longleftrightarrow \Psi_{4}(x, \vec{a}, \vec{z})\right)$, whence

$$
d \cap\{x \mid \Phi(x, \vec{a})\}=d \cap\left\{x \mid \Psi_{4}(x, \vec{a}, \vec{z})\right\} \in V
$$

8.11 COROLlary " $x \in \mathcal{S}(y)$ " is $\Delta_{1}^{\mathrm{DS}}$.

8.12 LEMMA (DS) $z \subseteq \mathcal{S}(y) \Longleftrightarrow \exists c[\forall w: \in z w \subseteq y \& \forall w: \in z \exists f: \in c \exists n: \in$ $\omega f: n \longleftrightarrow w]$.

Proof : Take $c=\mathcal{S}(y \times \omega)$.

8.13 CoROllary " $z=\mathcal{S}(y)$ " is $\Delta_{1}^{\mathrm{DS}}$.

Proof : The given formula is $\Sigma_{1}$ since $z=\mathcal{S}(y) \Longleftrightarrow z \subseteq \mathcal{S}(y) \& \Psi_{\mathcal{S}}(z, y)$; but then it will be $\Delta_{1}$ since $z=\mathcal{S}(y) \Longleftrightarrow \forall w(w \neq z \Longrightarrow w \neq \mathcal{S}(y))$.

8.14 REMARK The above discussion shows that the function $x \mapsto \mathcal{S}(x)$ is $\Sigma_{1}$ in $\operatorname{ReR}$ with $\omega \in V$ and $\Pi_{1}$ foundation.

8.15 MetatheOREM Every $\Pi_{1, \mathcal{S}}$ predicate is $\Pi_{1}^{\mathrm{DS}}$.

Proof : Consider a predicate of the form $\forall c \Phi(c, \vec{a})$ where $\Phi$ is $\Delta_{0, \mathcal{S}}$. We again use the Second Restricted Normal Form, which tells us that there is a $\Delta_{0}$ predicate $\Psi_{4}(c, a, \vec{z})$ and further variables $\vec{b}$ and $\vec{y}$, such that $\phi(c, a)$ is equivalent to $\Psi_{4}(c, a, \vec{z})$ provided finitely many conditions hold, of the form $z=\mathcal{S}(y) \& y=\bigcup^{\mathfrak{k}} b$ or $z=y \& y=\bigcup^{\ell} b$, and each $a$ and $c$ is either a member of or a subset of or equal to the corresponding $b$.

Thus, writing out a sample condition,

$$
\begin{aligned}
& \forall c \Phi(c, a) \Longleftrightarrow \\
& \forall c \overrightarrow{\forall b} \overrightarrow{\forall z} \overrightarrow{\forall y}[[[\underbrace{z=\mathcal{S}(y)}_{\Sigma_{1}} \& \underbrace{y=\bigcup^{\mathfrak{k}} b \& a \subseteq b}_{\Delta_{0}}] \& \ldots \& \underbrace{[\ldots]}_{\Sigma_{1}}] \Longrightarrow \underbrace{\Psi_{4}(c, a, \vec{z})}_{\Delta_{0}}]
\end{aligned}
$$

which is $\Pi_{1}$, as required.

8.16 COROLLARY DS proves that the graph $\mathcal{G}_{+}$of integer addition, or indeed of any partial recursive function, is a set.

Proof: To get the graph of addition, we would apply separation to $\omega \times(\omega \times \omega)$ to form the set of all triples such that there exists an attempt: prima facie $\Sigma_{1}$ or even $\Delta_{1}$ separation, given that attempts are unique (a fact that we 
have not proved). But the attempts are in $\mathcal{S}(\omega \times(\omega \times \omega))$ : so only $\Delta_{0, \mathcal{S}}$ is needed, which we know to be available in DS.

\section{DS with TCo}

8.17 Proposition (DS + TCo) $\operatorname{tcl}(x) \in V$.

Proof : fix x, and using TCo, let $u$ be a transitive set of which $x$ is a member. Using $\mathcal{S}(x) \in V$, let $a$ be the set $\mathcal{S}(u \times \omega)$.

Say that $f$ descends from $x$ to $y$ if

$$
\begin{aligned}
\operatorname{Fn}(f) \& \operatorname{Dom} f & \in \omega \& 2 \leqslant \operatorname{Dom} f \& f(0)=x \& \\
\forall k: & <\operatorname{Dom}(f)-1 f(k+1) \in f(k) \& f(\operatorname{Dom}(f)-1)=y .
\end{aligned}
$$

That is a $\Delta_{0}$ predicate of $f$, and each such $f$ is in $a$, so the class

$$
u \cap\{y \mid \exists f: \in a[f \text { descends from } x \text { to } y]\}
$$

is a set and is the desired transitive closure of $x$.

\section{Self-strengthening of GJS}

8.18 LEMMA (GJS) $\{\mathcal{S}(x) \mid x \in a\} \in V$.

Proof : Fix the set $a$. If $x \in a$ then $x \subseteq \bigcup a$, so $\mathcal{S}(x) \subseteq \mathcal{S}(\bigcup a)$. The desired set is the class

$$
\left\{\left.\mathcal{S}(\bigcup a) \cap\{y \mid y \subseteq x\}\right|_{x} x \in a\right\}
$$

which is a set by an application of $\mathrm{RR}^{+}$.

8.19 Corollary (GJS) $\left\{\left.\langle\mathcal{S}(\bigcup w), \mathcal{S}(w)\rangle\right|_{w} w \in b\right\} \in V$.

Proof : consider $\left\{\left.\mathcal{S}(v)\right|_{v} v \in a\right\} \times\left\{\left.\mathcal{S}(w)\right|_{w} w \in b\right\} \cap\left\{\left.(c, d)_{2}\right|_{c, d} \cup c=\right.$ $\bigcup \bigcup d\}$, taking $a=\left\{\left.\bigcup w\right|_{w} w \in b\right\}$.

8.20 PROPOSITION GJS proves $\Delta_{0, \mathcal{S}}$ rud replacement.

Proof : Aiming, in fact, for the extended form corresponding to $R R^{+}$, defined in 2.88, we must show that

$$
\forall x_{2} \forall x_{1} \exists w \overrightarrow{\forall v}: \in x_{1} \exists t: \in w \forall u\left(u \in t \Longleftrightarrow u \in x_{2} \& \Phi(u, \vec{v}),\right.
$$

where $\Phi$ is a $\Delta_{0, \mathcal{S}}$ formula with the free variables shown.

Suppose that there are $\mathfrak{m}+1$ quantifiers in $\Phi$. By the Second Restricted Normal Form, we know that there are new variables $y_{0}, \ldots, y_{\mathfrak{m}}, z_{0}, \ldots, z_{\mathfrak{m}}$ and a $\Delta_{0}$ formula $\Psi_{4}(u, \vec{v}, \vec{z})$ with the free variables shown, such that

$$
\begin{aligned}
\mathrm{DB}_{0} \vdash u \in x_{2} \& v \in x_{1} \&\left\langle\text { conditions on } \vec{z}, \vec{y}, x_{1}, \text { and } x_{2}\right\rangle \Longrightarrow \\
\Longrightarrow\left[\Phi(u, \vec{v}) \Longleftrightarrow \Psi_{4}(u, \vec{v}, \vec{z})\right],
\end{aligned}
$$


where there are $\mathfrak{m}+1$ conditions, each of one of the four following types, according to the quantifier structure of $\Phi$ :

$$
\begin{aligned}
& {\left[z=y \& y=\bigcup^{\mathfrak{l}+1} x_{2}\right] ;\left[z=\mathcal{S}(y) \& y=\bigcup^{\mathfrak{l}+1} x_{2}\right]} \\
& {\left[z=y \& y=\bigcup^{\mathfrak{l}+1} x_{1}\right] ;\left[z=\mathcal{S}(y) \& y=\bigcup^{\mathfrak{l}+1} x_{1}\right]}
\end{aligned}
$$

A slight extension of $R R^{+}$would tell us that

$$
\forall x_{2} \forall x_{1} \exists w \overrightarrow{\forall z}: \in A \overrightarrow{\forall v}: \in x_{1} \exists t: \in w \forall u\left(u \in t \Longleftrightarrow u \in x_{2} \& \Psi_{4}(u, \vec{v}, \vec{z})\right),
$$

where $A$ is a certain class, provably a set containing at most $\mathfrak{m}+1$ elements, namely the values of the form $\bigcup^{\mathfrak{l}+1} x_{2}$ or $\mathcal{S}\left(\bigcup^{\mathfrak{l}+1} x_{2}\right)$ given to the $z^{\prime}$ s by the conditions.

To show that, fix $x_{2}$. If we write $x_{3}$ for $x_{1} \cup A$, then by $R R^{+}$, we may deduce that

$$
\exists w \overrightarrow{\forall v}: \in x_{3} \forall z: \in x_{3} \exists t: \in w \forall u\left(u \in t \Longleftrightarrow u \in x_{2} \& \Psi_{4}(u, \vec{v}, \vec{z})\right),
$$

whence

$$
\exists w \forall \vec{v}: \in x_{3} \forall z: \in x_{3} \exists t: \in w \forall u\left(u \in t \Longleftrightarrow u \in x_{2} \& \Phi(u, \vec{v})\right) .
$$

We may now cut this $w$ down to exactly the one we want by applying $\Delta_{0, \mathcal{S}}$ separation.

\section{Self-strengthening of fReRS}

8.21 Proposition fReRS proves flat $\Delta_{0, \mathcal{S}}$ replacement.

Proof : We must show that

$\forall x: \in u \exists ! d[\Phi(x, d) \& d \subseteq e] \Longrightarrow \exists v \forall d[d \in v \Longleftrightarrow \exists x: \in u[\Phi(x, d) \& d \subseteq e]]$

where $\Phi$ is a $\Delta_{0, \mathcal{S}}$ formula with the two free variables shown.

Suppose that there are $\mathfrak{m}+1$ quantifiers in $\Phi$. By the Second Restricted Normal Form, we know that there are new variables $y_{0}, \ldots, y_{\mathfrak{m}}, z_{0}, \ldots, z_{\mathfrak{m}}$ and a $\Delta_{0}$ formula $\Psi_{4}(x, d, \vec{z})$ with $\mathfrak{m}+3$ free variables, such that

$$
\begin{aligned}
\mathrm{DB}_{0} \vdash x \in u \& d \subseteq e \&\langle\text { conditions on } \vec{z}, \vec{y}, u \text {, and } e\rangle \Longrightarrow \\
{\left[\Phi(x, d) \Longleftrightarrow \Psi_{4}(x, d, \vec{z})\right], }
\end{aligned}
$$


where there are $\mathfrak{m}+1$ conditions, each of one of the four following types, according to the quantifier structure of $\Phi$ :

$$
\begin{gathered}
{\left[z=y \& y=\bigcup^{\mathfrak{l}+1} u\right] ;\left[z=\mathcal{S}(y) \& y=\bigcup^{\mathfrak{l}+1} u\right]} \\
{\left[z=y \& y=\bigcup^{\mathfrak{l}} e\right] ;\left[z=\mathcal{S}(y) \& y=\bigcup^{\mathfrak{l}} e\right] .}
\end{gathered}
$$

Fix $u$ and $e$; then, using $\forall x \mathcal{S}(x) \in V$, the conditions will give fixed values to the $y$ 's and $z$ 's; for those values we shall have that for $x \in u$ and $d \subseteq e, \Phi(x, d) \Longleftrightarrow \Psi_{4}(x, d, \vec{z})$.

Suppose now that $\forall x: \in u \exists ! d[\Phi(x, d) \& d \subseteq e]$; then

$$
\forall x: \in u \exists ! d\left[\Psi_{4}(x, d, \vec{z}) \& d \subseteq e\right] .
$$

We appeal to the extended form of (BdR) proved as Proposition 2.101, to deduce that

$$
\exists v \forall d\left[d \in v \Longleftrightarrow \exists x: \in u\left[\Psi_{4}(x, d, \vec{z}) \& d \subseteq e\right]\right],
$$

whence

$$
\exists v \forall d[d \in v \Longleftrightarrow \exists x: \in u[\Phi(x, d) \& d \subseteq e]] .
$$

8.22 REMARK The system fReRS proves appropriate for the development of the definition of forcing, and fReCS might be the weakest system persistent under set-generic extensions: see [M5].

8.23 Problem Is fReR with the ancestral added adequate for rud rec rels?

\section{Self-strengthening of ReR}

8.24 LEMma (ReR) All instances of $\Delta_{0}$ replacement where, as in 2.101, $\varphi$ is allowed to have further free variables.

Proof : Suppose that $\mathfrak{A}$ is $\Delta_{0}$ and that $\forall x: \in u \exists ! y \mathfrak{A}(x, y, w)$. Let $u_{1}=$ $u \times\{w\}$. Then

$$
\forall x: \in u_{1} \exists ! y \underbrace{\mathfrak{A}(\operatorname{left}(x), y, \operatorname{right}(x))}_{\Delta_{0}^{\mathrm{S}_{0}}} .
$$

So applying $\Delta_{0}$ replacement, we get $\exists v \forall y(y \in v$ if and only if

$$
\exists x: \in u_{1} \mathfrak{A}(\operatorname{left}(x), y, \operatorname{right}(x)),
$$

which in turn is equivalent to $\exists x: \in u \mathfrak{A}(x, y, w)$, as required.

8.25 Proposition ReRI proves each instance of $\Delta_{0, \mathcal{S}}$ replacement. 
Proof : The argument given for 8.21 adapts easily, using the Lemma.

8.26 Problem Does ReR prove $\mathcal{S}(x) \in V$ ? the idea being that if there is an infinite set, then one ought to be able to prove that $\omega$ exists, and thence that $\mathcal{S}(x) \in V$; and if all sets are finite a proof of $\mathcal{S}(x) \in V$ will be provided by Proposition 2.13 .

We pause to establish two results concerning the sets $Z(a)$ defined in [M1], whose definition was recalled in our discussion of Model 7.

8.27 Definition We write " $f$ attempts $Z(a)$ at $n$ " for the $\Delta_{0, \mathcal{S}}$ formula

$$
\begin{aligned}
\operatorname{Fn}(f) \& \operatorname{Dom}(f) & =n+1 \& f(0)=\varnothing \& \\
& \& \forall k: \in n(f(k+1)=\mathcal{S}(f(k)) \cup\{a\} \backslash\{\varnothing\}) .
\end{aligned}
$$

8.28 Proposition (ReRI) $\forall a: \omega \longrightarrow 2, Z(a)$ exists.

Proof: Fix $a$. Note that if $\operatorname{Fn}(f)$ then

$$
x=\mathcal{S}(f(k)) \Longleftrightarrow \underbrace{\exists y: \in \bigcup \bigcup(f)(y, k)_{2} \in f \& x=\mathcal{S}(y)}_{\Delta_{0, \mathcal{S}}} .
$$

Hence we may assert that

$$
\forall n: \in \omega \exists f(f \text { attempts } Z(a) \text { at } n) ;
$$

for the class of $n$ for which the assertion fails is $\Pi_{1, \mathcal{S}}$ and therefore by ??? has, if non-empty, a minimal element, necessarily a successor; which can rapidly be refuted.

For each $n$, there can be at most one such $f$, so by $\Delta_{0, \mathcal{S}}$ replacement, the set of such $f$ exists; its union will be a function, of which the class $Z(a)$ is the image and therefore a set.

8.29 Definition Let $\Psi(x, a)$ be the $\Delta_{0, \mathcal{S}}$ formula

$$
\begin{array}{r}
a \in x \& \forall b: \in x[\{b\} \in x \&(b \in \mathcal{S}(x) \vee b=a) \&(b=\varnothing \Longrightarrow b=a)] \& \\
\& \forall s: \in \mathcal{S}(x)[s \neq \varnothing \Longrightarrow s \in x] .
\end{array}
$$

8.30 LEMMA (ReRI) $Z(a) \in V \Longrightarrow x=Z(a) \Longleftrightarrow \Psi(x, a)$.

Proof : It is readily checked that $x=Z(a) \Longrightarrow \Psi(x, a)$.

Suppose that $Z(a) \in V$ and that $\Psi(x, a)$. Let $c=\mathcal{S}(Z(a) \times \omega)$. Then

$$
\left\{n \mid Z_{n}(a) \nsubseteq x\right\}=\{n \mid \underbrace{\exists f: \in b f \text { attempts } Z(a) \text { at } n \& f(n) \nsubseteq x}_{\Delta_{0, \mathcal{S}}}\} ;
$$


A. R. D. MATHIAS

$\Pi_{1}$ foundation would yield a minimal element of that class, if non-empty; but $Z_{0}(a)=\varnothing \subseteq x$, and it is easily checked that $\Psi(x, a) \& Z_{n}(a) \subseteq x \Longrightarrow$ $Z_{n+1} \subseteq x$. Thus $Z(a) \subseteq x$.

If $x \not \subset Z(a)$, let $y$ be an $\in$-minimal element of $x \backslash Z(a)$. Then $y \neq \varnothing$, $y \in \mathcal{S}(x)$ and $y \subseteq Z(a)$. Hence $\forall z: \in y \exists ! n: \in \omega(\underbrace{z \in Z_{n+1}(a) \& z \notin Z_{n}(a)}_{\Delta_{0, \mathcal{S}}})$; the class of such $n$ 's is therefore a set, which is finite and therefore bounded in $\omega$; so $\exists m: \in \omega y \subseteq Z_{m}(a)$, whence $y \in Z_{m+1}(a)$, contradicting $y \notin Z(a)$.

$\dashv(8.30)$

8.31 Corollary (ReRI) " $x=Z(a)$ " is $\Delta_{0, \mathcal{S}}$.

8.32 Proposition (ReRI) $\forall b_{\subseteq} \omega_{2}\{Z(a) \mid a \in b\} \in V$.

Proof : Fix $b$. Then $\forall a: \in b \exists ! x \underbrace{x=Z(a)}_{\Delta_{0, \mathcal{S}}}$; apply $\Delta_{0, \mathcal{S}}$ replacement to complete the proof.

\section{Self-strengthening of KPI}

8.33 Proposition KPI proves every instance of $\Delta_{0, \mathcal{S}}$ collection.

Proof : We may either use Remark 8.30 or else Metatheorem 8.31, which implies that in the context of $\mathrm{KPI}$, every $\Delta_{0, \mathcal{S}}$ formula is equivalent to a $\Sigma_{1}$ one; but it is well-known that KP is self-strengthening to $\Sigma_{1}$ collection.

8.34 PROBLEM Here the case is even stronger; in KP rank is definable and the rank of an infinite set must be at least $\omega$. Then with infinity you get $\mathcal{S}(x) \in V$. So the Proposition ought to be provable in KP alone.

\section{THE GANDY SEQUENCE}

In this section we wish to assess the relative strength of the enhanced theories DS, etc.

9.0 Proposition There is a model of DS plus $\mathbf{H F} \in V$ in which $\mathrm{GJ}$ is false.

Proof: The model $\mathbf{M}_{7}$ will do. We have to prove that $\mathcal{S}(x) \in V$ is true in $\mathbf{M}_{7}$. Note that any non-empty finite set must have successor rank. So if $u$ is transitive and contains only finitely many transitive sets of limit rank, then $u \cup \mathcal{S}(u) \cup\{\mathcal{S}(u)\}$ will have the same property. That suffices. $\quad \dashv(9.0)$

\section{GJS in $\mathbf{L}$ and $\mathbf{J}$}

Now we wish to verify that GJS is true in every $L_{\lambda}(\lambda=\bigcup \lambda>\omega)$ and $J_{\alpha}(\alpha>1)$. 
9.1 Proposition " $\mathcal{S}(x) \in V$ " is true in every $L_{\lambda}$.

Proof : evidently so for $\lambda=\omega$; thereafter we have languages. Given $x \in L_{\zeta}$, all its finite subsets will be in $L_{\zeta+1}$, and the set of them will be in $L_{\zeta+2}$.

9.2 Proposition " $\mathcal{S}(x) \in V$ " is true in every $J_{\alpha}$.

9.3 LEMMA The sequence $\left\langle[\zeta]^{<\omega} \mid \zeta<\omega \alpha\right\rangle$ is uniformly $\Sigma_{1}$ over every $J_{\alpha}$. Proof : by a rud recursion.

The $S_{\omega \beta+k}$ used in the next proof may be defined as in Dodd's book, or one might use the sets corresponding to the $T_{n}$ defined in the proof of Proposition 9.7.

9.4 LEMMA In each $J_{\alpha}$, to every set $x$ there is an ordinal $\lambda$ and a surjection $f: \lambda \stackrel{\text { onto }}{\longrightarrow} x$.

Proof: In $J_{\alpha}$ each set is a member of some $S_{\omega \beta+k}$, with $\beta<\alpha$, so we may derive the lemma from [Do], chapter 1 , section 2 , Lemma 2.42 on page 20 , which Dodd proves within his theory $R_{\omega}^{+}$that he introduces on page 12 . In our terms that is the theory GJ (without TCo ?) plus a version of "V = L" plus certain instances of the scheme of full foundation. He shows though that each $J_{\alpha}$ models this theory: see his Lemma 2.21 on page $14 . \quad \dashv(9.4)$

Proof of the proposition: let $f \in J_{\alpha}$ be a surjection from $\zeta$ to $x$. Then $\mathcal{S}(x)=\{f " a \mid a \in \mathcal{S}(\zeta)\}$.

9.5 Proposition Let $\lambda$ be a limit ordinal. Then $L_{\lambda}$ models $(R R)$.

Proof: For if $x$ is in $L_{\zeta}$ each of the $x \cap\{u \mid \phi(u, \vec{v})\}$ is in $L_{\zeta+1}$ and the set of them is in $L_{\zeta+2}$.

9.6 Proposition $\mathbf{H F}=L_{\omega}=J_{1}$, and hence is a member of $L_{\omega+\nu}$ and of $J_{1+\nu}$ for each $\nu>0$.

\section{Model 14: of GJS without fReR}

9.7 Proposition There is a model of GJS plus $\mathbf{H F} \in V$ in which fReR is false.

Proof: Such a model is $J_{2}$. Here we shall use the existence of our single rudimentary function $g$ of Definition 2.75 that for any transitive set $u$ generates the rudimentary closure of $u \cup\{u\}$. It has these properties: every element of $\mathbb{T}(u)$ is a subset of $u$ and is of the form $S(u ; x, y)$, where $S$ is one of our list $S_{0}, \ldots S_{9}$ of ten rudimentary functions, and $x, y \in u$. Similarly each element of $\mathbb{T}(\mathbb{T}(u))$ is a subset of $\mathbb{T}(u)$ and is of the form $S(\mathbb{T}(u) ; x, y)$, where $x$ and $y$ are members of $\mathbb{T}(u)$. 
Our function $\mathbb{T}$ differs slightly from those used by Jensen, Devlin and Dodd, and so we make a corresponding change of notation. We write $T_{0}$ for $J_{1}$, and successively $T_{n+1}$ for $\mathbb{T}\left(T_{n}\right)$. Then $J_{2}=\bigcup_{n \in \omega} T_{n}$.

Our intention is to build a calculus of terms, using names $\dot{S}_{i}$ for $S_{i}$ in that finite list, and allowing as arguments names for the various $T_{n}$ and their members. We define the class of terms recursively. $\mathcal{W}_{0}$ is to comprise symbols for the members of $J_{1}$. Having formed $\mathcal{W}_{n}$, we take a new symbol $\tau_{n}$ for $T_{n}$, and let $\mathcal{W}_{n+1}$ be the set of words of the form $\left.\dot{S}_{i} \dot{(} \tau_{n} ; v, w\right)$ where $v$ and $w$ are words in $W_{n}, 0 \leqslant i \leqslant 9$, and $\dot{(a n d} \dot{)}$ are the parentheses of the formal language we are developing.

Thus $\mathcal{W}_{1}$ comprises words of the form $\dot{S}\left(\tau_{1} ; x, y\right)$ where $x$ and $y$ are in $\mathcal{W}_{0}$

We suppose that our symbols are coded so that $\mathcal{W}_{n} \subseteq \omega \subseteq J_{1}=\mathbf{H F}$, and that the $\mathcal{W}_{n}$ are pairwise disjoint, and that the coding has been done in some reasonable recursive way, so that in particular the map $k \mapsto\ulcorner k\urcorner$ is recursive with recursive inverse, and that there are recursive enumerations $\left(w_{k}^{n}\right)_{k}$ of the words in $\mathcal{W}_{n}$.

Let $\mathcal{E}_{n}$ be the evaluation function of these words: so that $\mathcal{E}_{n}\left[\mathcal{W}_{n}\right]$ is our version $T_{n}$, defined in greater detail below, of the Jensen partial stage $S_{n}\left(J_{1}\right)$ on the way to building $J_{2}$.

Let $\mathcal{M}_{n}$ be the relation on $\omega$ defined by

$$
\mathcal{M}_{n}(w, v) \Longleftrightarrow w \in \mathcal{W}_{n} \& v \in \mathcal{W}_{n} \& \mathcal{E}_{n}(w) \in \mathcal{E}_{n}(v) .
$$

Let $\mathcal{Q}_{n}$ be the relation on $\mathcal{W}_{n}$ defined by

$$
\mathcal{Q}_{n}(w, v) \Longleftrightarrow w \in \mathcal{W}_{n} \& v \in \mathcal{W}_{n} \& \mathcal{E}_{n}(w)=\mathcal{E}_{n}(v) .
$$

9.8 REMARK In our context, of full extensionality, $\mathcal{Q}_{n}$ will of course be rudimentary in $\mathcal{M}_{n}$, and might therefore be dropped from this discussion; but with possible applications of the present argument in a non-extensional context in mind, we keep both predicates in play.

9.9 LEMma There are rudimentary functions $G$ and $H$ such that

$$
\mathcal{M}_{n+1}=G\left(\mathcal{M}_{n}, \mathcal{Q}_{n}\right) \& \mathcal{Q}_{n+1}=H\left(\mathcal{M}_{n}, \mathcal{Q}_{n}\right)
$$

Proof: We examine the passage from one stage to the next in greater detail. We have a set $W$ of words and an evaluation $\mathcal{E}$ for those words, such that $\mathcal{E}[W]=U$, a transitive set. We add a term $\tau$ to the language to denote $U$. We define a new set of words thus:

$$
W^{+}=\left\{\dot{S}_{i}(\tau ; v, w) \mid 0 \leqslant i \leqslant 9, v \in W, w \in W\right\} .
$$


We define an evaluation $\mathcal{E}^{+}$of the words in $W^{+}$thus:

$$
\mathcal{E}^{+}\left(\dot{S}_{i}(\tau ; v, w)\right)=S_{i}(U ; \mathcal{E}(v), \mathcal{E}(w)) .
$$

The evaluation of course takes place in the set theoretical universe. We wish to show that it can be carried out at a more formal level.

We define relations $\mathcal{M}, \mathcal{Q}$ on $W$, and $\mathcal{M}^{+}, \mathcal{Q}^{+}$on $W^{+}$, and we shall show that the second pair are uniformly rudimentary in the first pair.

\subsection{DEFINITION}

$$
\begin{aligned}
& \mathcal{M}(v, w) \Longleftrightarrow \mathrm{df} \mathcal{E}(v) \in \mathcal{E}(w) \\
& \mathcal{Q}(v, w) \Longleftrightarrow \mathrm{df} \\
& \mathcal{E}(v)=\mathcal{E}(w)
\end{aligned}
$$

and similarly

$$
\begin{aligned}
& \mathcal{M}^{+}\left(v^{+}, w^{+}\right) \Longleftrightarrow \mathcal{d f} \mathcal{E}^{+}\left(v^{+}\right) \in \mathcal{E}^{+}\left(w^{+}\right) \\
& \mathcal{Q}^{+}\left(v^{+}, w^{+}\right) \Longleftrightarrow{ }_{\mathrm{df}} \mathcal{E}^{+}\left(v^{+}\right)=\mathcal{E}^{+}\left(w^{+}\right)
\end{aligned}
$$

9.11 REMARK Let $U^{+}=\mathcal{E}^{+}\left(W^{+}\right)$: then $U^{+}=\mathbb{T}(U)$.

The essential observations are that each evaluation $\mathcal{E}^{+}\left(v^{+}\right)$of a word in $W^{+}$will be a subset of $U$, and therefore quantification over $U$ suffices for comparing one evaluation with another; and that describing the functions involved is always $\Delta_{0}$.

9.12 LEMMA For $z \in W$ and $w^{+}$a word in $W^{+}$, the relation $\mathcal{E}(z) \in \mathcal{E}^{+}\left(w^{+}\right)$ is (uniformly) rudimentary in $W, \mathcal{M}$ and $\mathcal{Q}$.

Proof : Let $w^{+}$be $\dot{S}_{p}\left(\tau ; w_{1}, w_{2}\right)$. If, say, $p=2$, we shall have

$$
\mathcal{E}(z) \in \mathcal{E}^{+}\left(w^{+}\right) \Longleftrightarrow \exists w_{3}: \in W\left(\mathcal{M}\left(z, w_{3}\right) \& \mathcal{M}\left(w_{3}, w_{1}\right)\right)
$$

For the general case, the function $S_{i}$ being rudimentary, $z \in S(u ; x, y)$ will be a $\Delta_{0}$ predicate of $z, u, x$ and $y$; rewrite that predicate by requiring all bound variables to be restricted to members of $W$, and as for atomic formulæ, replace $a=b$ by $\mathcal{Q}(a, b)$ and $a \in b$ by $\mathcal{M}(a, b)$. Note that $u$ only occurs in contexts such as $u \cap R_{i}(x)$ or $u \cap R_{j}(x, y)$ and hence when written out, only in atomic formulæ of the form $a \in u$; which will always be evaluated as true, as $\tau$ denotes $T$, the set of evaluations of the variables.

Given that lemma, the relation $\mathcal{Q}^{+}\left(v^{+}, w^{+}\right)$being equivalent to $\forall z: \in$ $W\left(\mathcal{E}(z) \in \mathcal{E}^{+}\left(v^{+}\right) \Longleftrightarrow \mathcal{E}(z) \in \mathcal{E}^{+}\left(w^{+}\right)\right.$, will be rudimentary in $W, \mathcal{M}$ and $\mathcal{Q}$.

Now for $\mathcal{M}^{+}$. 
9.13 LEMma For $z \in W$ and $w^{+}$a word in $W^{+}$, the relation $\mathcal{E}(z)=\mathcal{E}^{+}\left(w^{+}\right)$ is (uniformly) rudimentary in $W, \mathcal{M}$ and $\mathcal{Q}$.

Proof $: \mathcal{E}(z)=\mathcal{E}^{+}\left(w^{+}\right) \Longleftrightarrow \forall y: \in W\left[\mathcal{E}(y) \in \mathcal{E}^{+}\left(w^{+}\right) \Longleftrightarrow \mathcal{M}(y, z)\right]$, since $\mathcal{M}(y, z) \Longleftrightarrow \mathcal{E}(y) \in \mathcal{E}(z)$.

Now $\mathcal{M}^{+}\left(v^{+}, w^{+}\right) \Longleftrightarrow \exists z: \in W \mathcal{E}^{+}\left(v^{+}\right)=\mathcal{E}(z) \& \mathcal{E}(z) \in \mathcal{E}^{+}\left(w^{+}\right)$, and so $\mathcal{M}^{+}$is rudimentary in $W, \mathcal{M}$ and $\mathcal{Q}$ by the last two lemmata.

Our lemma is now established by the uniformity of the above discussion. $\dashv(9 \cdot 10)$

Hence we may write a formula $\Phi(n, Z)$ which says that $Z$, a subset of $\omega$ codes the sequences $\left\langle\mathcal{M}_{m} \mid 1 \leqslant m \leqslant n\right\rangle$ and $\left\langle\mathcal{Q}_{m} \mid 1 \leqslant m \leqslant n\right\rangle$; once we have fixed our coding, there will be a unique $Z$, call it $Z_{n}$ that does that.

All the above can be carried out in $J_{2}$. Suppose that fReR were true there. Then there would be a set $A$ containing all the $Z_{n}$ 's. But uniformly from $Z_{n}$ we can form the set $X_{n}$ defined by

$$
X_{n}=_{\mathrm{df}}\left\{k \in \omega \mid \neg \mathcal{M}_{n}\left(\ulcorner k\urcorner, w_{k}^{n}\right)\right\},
$$

where $\ulcorner k\urcorner$ is our canonical symbol for $k$ (so that $\mathcal{E}_{n}(\ulcorner k\urcorner)=k$ for every $n$ )and $\left(w_{k}^{n}\right)_{k}$ is a recursive enumeration of $\mathcal{W}_{n}$. Hence there will be some $\ell$ such that $T_{\ell}$ contains all the $X_{n}$ 's. We now get a contradiction, for $X_{\ell}$ itself cannot be a member of $T_{\ell}$. If it were, it would for some $k$ be the evaluation $\mathcal{E}_{\ell}\left(w_{k}^{\ell}\right)$ of some word $w_{k}^{\ell}$. But then for that $k$,

$$
k \in X_{\ell} \Longleftrightarrow \mathcal{M}_{\ell}\left(\ulcorner k\urcorner, w_{k}^{\ell}\right) \Longleftrightarrow k \notin X_{\ell} .
$$

9.14 Proposition There is a model of fReCS in which ReR is false.

Proof : $V_{\omega+\omega}$; alternatively, $V_{\omega+\omega} \cap \mathbf{H C}$.

\section{Model 15: of $\mathbf{Z}$ without restricted rank-bounded replacement}

We apply the pivotal idea of Zarach [Z] to the model-building of [M1, section 4]. We have above recalled the definition of $Z(a)$; we shall use these further definitions from [M1]:

9.15 Definition $b_{0}(n)=n ; b_{k+1}(n)=2^{b_{k}(n)} ; \mathcal{F}$ is the family of functions from $\omega$ to $\omega$ that are dominated by some $b_{k}$; for $u$ transitive, $f_{u}^{a}(n)=$ $\overline{\overline{u \cap Z_{n}(a)}} ; \mathcal{T}^{a}=\left\{u \mid \bigcup u \subseteq u \& f_{u}^{a} \in \mathcal{F}\right\} . T(a)=\operatorname{tcl}(a) \cup Z(a) \cup\{Z(a)\}$.

9.16 LEMMA (i) If $Z(b)$ is in $u$, transitive, then $f_{u}^{b}$ is not in $\mathcal{F}$, so $u$ is not in $\mathcal{T}^{b}$.

(ii) For $a \neq b, Z(b) \in T(b) \in \mathcal{T}^{a}$. 
Proof : as in the proof of [M1, Theorem 4.8], but note that (ii) of the present lemma corrects a slip in the last sentence of the first paragraph of that proof.

Now let $A$ be an infinite subset of ${ }^{\omega_{2}}$. Let $I$ be a proper ideal on $A$ extending the Fréchet ideal of all finite subsets of $A$. For $s \in I$, let $A^{s}=\bigcap\left\{\mathcal{T}^{a} \mid a \in A \backslash s\right\}$, and let $M^{s}=\bigcup A^{s}$. Finally, set $\mathbf{M}_{15}=\bigcup_{s} M^{s}$.

9.17 Now $M^{s} \cup M^{t} \subseteq M^{s \cup t}$, since $s_{1} \subseteq s_{2} \Longrightarrow A^{s_{1}} \subseteq A^{s_{2}}$, so AxPair will hold in M. Further, $b \in s \Longrightarrow T(b) \in A^{s}$, so $Z(b) \in M^{s}$, and so each $Z(b)$ is in $\mathcal{M}=\bigcup_{s} M^{s}$.

Indeed, $\mathbf{M}_{15}$ is a supertransitive model of $\mathbf{Z}$ containing all ordinals, in which full flat collection holds, and TCo; and in which every set has a rank.

But $\{Z(b) \mid b \in A\}$ is not in $\mathbf{M}_{15}$; if it were a member of $u$, transitive and in $A^{s}$, take $a \in A \backslash s$; then $f_{u}^{a}$ is not in $\mathcal{F}$ so $u \notin \mathcal{T}^{a}$ and therefore not in $A^{s}$. Hence by Proposition $8.32, \mathbf{M}_{15}$ is not a model of ReRI; and indeed the failure is one of rank-bounded replacement in that all the $Z(a)$ are of $\operatorname{rank} \omega+\omega$.

$\dashv(9.17)$

9.18 As Z extends full separation and proves $\mathcal{S}(x) \in V$ and full flat collection, the above model shows in particular that restricted replacement is not provable in fReRS.

9.19 As ReRI proves $\mathcal{S}(x) \in V$ and $\mathbf{H F} \in V$, Zarach's model suffices to show that that theory does not prove restricted collection.

\section{MENDING THE FLAWS IN DEVLIN'S BOOK}

We turn now to a discussion of the flaws in Devlin's book Constructibility to which attention was drawn in Stanley's review mentioned in a previous section. The problems are chiefly confined to section 9 of Chapter I and section 1 of Chapter VI.

We begin with a brief summary of Devlin's notation; we then mention a general problem, not, alas, confined to Devlin's book; then we work through Section 9 of Chapter I, where the system BS is introduced as the intended vehicle for the stream of thought in that section: we point out places where BS is inadequate, and places where, with some correction, it suffices; then we do the same for Section 1 of Chapter VI; then we go through again, seeing to what extent our system DS proves to be adequate; then again, using instead the system GJI $+\Pi_{1}$ foundation.

\section{Some comments on Devlin's notation}

On page 9: an $n$-tuple is introduced as a Wiener-Kuratowski one. In a familiar tradition, a function is treated as a subset of its image $\times$ its 
domain. On page 11: a sequence is defined as a function whose domain is an ordinal; so a finite sequence is one whose domain is a finite ordinal; a natural number is a finite ordinal.

Thus an $n$-sequence is an object of cardinality $n$ consisting of ordered pairs of which the second elements form a finite initial segment of the ordinals. The 4 -sequence $\langle 0,1,2,3\rangle$ is written thus to distinguish it from the (WK) 4-tuple $(0,1,2,3)_{4}$.

We maintain our policy of writing ${ }^{3} X$ for the set of 3 -sequences of members of $X ; X^{3}$ for the set of WK 3-tuples of members of $X$; thus $\omega^{3}=\omega \times(\omega \times \omega)$.

10.0 REMARK Devlin makes no distinction between $(X \times X) \times X$ and $X \times(X \times X)$, writing both as $X^{3}$. With weak systems that is scarcely satisfactory, since the variant given of Model 4, using weak right WK-rank, is a model of $\operatorname{ReS}_{0}$ which contains $(\omega \times \omega) \times \omega$ but not $\omega \times(\omega \times \omega)$; and, following the lead of Model 9, we can get models of BS containing either, but not both, of ${ }^{3}(\omega \times(\omega \times \omega))$ and ${ }^{3}((\omega \times \omega) \times \omega)$.

As for abbreviations of lists of variables, Devlin follows the useful convention that $\vec{x} \in A$ abbreviates $x_{1} \in A \& \ldots \& x_{n} \in A$, whereas $(\vec{x}) \in A$ indicates that the corresponding WK $n$-tuple is in $A$.

\section{The problem of levels of language}

There is an ambiguity over the meaning of $\Delta_{0}$ (which Devlin calls $\Sigma_{0}$ ). Devlin on page 230 writes:

In class terms a function is $\Sigma_{0}$ if of the form $\{(y, \vec{x}) \mid \Phi(y, \vec{x})\}$ where $\Phi$ is a $\Sigma_{0}$ formula of LST. In set-theoretic terms a function $f$ is said to be $\Sigma_{0}$ if there is a $\Sigma_{0}$ formula $\phi$ of $\mathcal{L}$ such that for any $\vec{x}, y$, if $M$ is a transitive set such that $\vec{x}, y \in M$, then

$$
f(\vec{x})=y \Longleftrightarrow \mid=M \varphi(\stackrel{\circ}{y}, \vec{x}) .
$$

10.1 REMARK The second definition has the advantage that one can then legitimately quantify over all $\varphi$; but the disadvantage that the definition collapses if TCo is false; whereas the first definition is still operational. Thus Devlin's remark that the two definitions are "equivalent" is dangerous.

\section{Errors in Chapter I}

\section{Definition of Finseq}

10.2 REMARK The definition of Finseq might not be as intended, on [Dev, page 33]; what is written is that members of Finseq are functions with 
domain a non-empty bounded subset of $\omega$ (possibly not a proper initial segment of $\omega)$.

We shall suppose that the definition has been corrected to mean that members of Finseq are functions with domain a non-empty bounded initial segment of $\omega$; that is still $\Delta_{0}$, so no harm has been done.

Lemmata 9.1 and 9.2 are correct.

The trouble starts on page 34 , with the formula $F_{\wedge}(\theta, \phi, \psi)$ : in its definition the clause "Dom $(\theta)=\operatorname{Dom}(\phi)+\operatorname{Dom}(\psi)+3$ " occurs. Thus addition of natural numbers is being used, in order to define concatenation.

\section{Lemma 9.3: " $F_{\wedge}$ is $\Delta_{0}$ "}

Though the other parts of Lemma 9.3 are correct as stated, that statement is false - Solovay has remarked that that can be seen by EhrenfeuchtFraissé games.

Its falsehood may indeed be established by arguments from Gandy's paper, where he proves (by a quantifier elimination argument, which is what, presumably, Solovay had in mind) that every $\Delta_{0}$ subset of $\omega$ is finite or cofinite; from that he shows that the graph of addition is not $\Delta_{0}$, and further deduces that the graph of concatenation is not $\Delta_{0}$.

Suppose we consider a language which accepts as atomic formulæ all finite constant sequences of $*$ 's. Note that each such sequence is expressible as $\{*\} \times n$ for some $n$.

Let $\theta_{n, k}$ be the term

$\left(\{*\} \times(k+3) \backslash\left\{(*, 0)_{2},(*, n+1)_{2},(*, k+2)_{2}\right\}\right) \cup\left\{(\dot{(}, 0)_{2},(\wedge, n+1)_{2},(\dot{)}, k+2)_{2}\right\}$

where $\dot{(}, \wedge$ and $\dot{)}$ code the left parenthesis, conjunctive connective and right parenthesis of the formal language.

Then $k=n+m \Longleftrightarrow F_{\wedge}\left(\theta_{n, k},\{*\} \times n,\{*\} \times m\right)$, and thus $F_{\wedge}$ cannot be $\Delta_{0}$ as the graph of addition is not.

\section{Complexity of $F_{\wedge}$}

10.3 Proposition $F_{\wedge}$ is $\Delta_{1}^{\mathrm{BS}}$ 
Proof : We propose to revise the definition of $F_{\wedge}$ as follows:

$$
\begin{aligned}
& F_{\wedge}(\theta, \varphi, \psi) \Longleftrightarrow \text { Finseq }(\theta) \& \text { Finseq }(\varphi) \& \operatorname{Finseq}(\psi) \& \\
& \& \exists f[[F n(f) \& \operatorname{Dom}(f)=\operatorname{Dom}(\theta) \times \operatorname{Dom}(\theta) \& \\
&\& f \text { is an attempt at addition }] \\
& \&[\operatorname{Dom}(\theta)=f(\operatorname{Dom}(\varphi)+1, \operatorname{Dom}(\psi)+1)+1 \& \\
& \&\left\langle\text { four incontrovertibly } \Delta_{0} \text { clauses }\right\rangle \& \\
&\& \forall i: \in \operatorname{Dom}(\psi)[\theta(f(\operatorname{Dom}(\varphi)+1, i+1)=\psi(0)]]] ;
\end{aligned}
$$

and because there is exactly one attempt at addition for a given finite domain, we may in the above replace the subformula $\exists f[[\ldots] \&[\ldots]]$ by $\forall f[[\ldots] \Longrightarrow[\ldots]]$, and obtain an equivalent formula which is $\Pi_{1}^{\mathrm{BS}}$.

\section{The definition of Build}

There is the following danger with the definition of Build: suppose that $\operatorname{Build}\left(\varphi, \psi_{1}\right)$. Now let $\psi_{2}$ result from $\psi_{1}$ by adding various formulæ to the sequence, keeping $\varphi$ always the last, and not using variables other than those in $\psi_{1}$; for example one might add many atomic formulæ; or one might interpolate the terms of some $\psi_{3}$ that builds some other formula, subject only to the condition on variables. Then $\psi_{2}$ also builds $\varphi$ according to the definition of Build: it might be that some minimality condition is needed, to the effect that every formula listed is actually a subformula of the formula being built.

\section{Lemma 9.4 "Build $(\varphi, \psi)$ is $\Delta_{0}$. "}

The proof is certainly invalid since it uses 9.3. The statement is suspect: suppose we add to the definition of Build extra clauses admitting the "formulæ" $\{*\} \times n$, as atomic: that would not change the $\Delta_{0}$ character of Build, as those clauses would be $\Delta_{0}$, even (by Gandy's proof that $\omega$ is $\mathrm{S}_{0^{-}}$ semi-suitable) when quantified over $n \in \omega$. Then for $\theta_{n, k}$ the term defined above,

$$
k=n+m \Longleftrightarrow \operatorname{Build}\left(\theta_{n, k},\left\langle\{*\} \times n,\{*\} \times m, \theta_{n, k}\right\rangle\right),
$$

and therefore Build (in the form modified to allow atomic wffs of the form $\{*\} \times n)$ cannot be $\Delta_{0}$ as the graph of addition is not.

As remarked above, Build might admit junk; if so, one must prove some "junk-excluding" clauses, such as $\left.\theta_{n, k} \neq\right\urcorner^{n}\{*\}$. 


\subsection{Proposition Build $(\varphi, \psi)$ is $\Delta_{1}^{\mathrm{BS}}$.}

Proof : use Proposition 10.3 and apply the principle of collection over finite sets given as Metatheorem 2.32.

\section{The formula Seq}

At the bottom of page 36 a formula $\operatorname{Seq}(u, a, n)$ is defined which expresses the statement that $u$ is the set of all finite sequences, of length less than $n$, of elements of $a$, and is correctly stated to be $\Sigma_{1}$. But this formula gives trouble in the proof of the next Lemma.

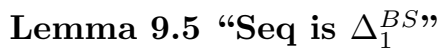

According to Solovay, the statement is false, "as may be seen using a forcing argument". I have been unable to demonstrate the falsity of the assertion using my present methods, but the model-building of previous sections will pin-point flaws in the argument.

In Model 6, there is no $u$ such that $\operatorname{Seq}(u, \omega, 4)$; so in that model the proposed $\Pi_{1}$ form of the definition is true of everything, and the proposed $\Sigma_{1}$ form is false of everything. So the equivalence is not a theorem of BS, and the proposed proof of I.9.5 cannot succeed.

In detail:

10.5 The first displayed formula in the proof of 9.5 asserts that

"it is clear from the definition of BS that:

$$
B S \vdash(\forall a)(\forall n \in \omega)(\exists u) \operatorname{Seq}(u, a, n) . "
$$

But that statement, on lines 5 and 6 of page 37 , is not a theorem of BS, as is shown by Model 9, in which there is no $u$ with $\operatorname{Seq}(u,\{\omega\} \times \omega, 4)$, or, indeed, by Model 6 , in which for no infinite $a$ is there a $u$ with $\operatorname{Seq}(u, \omega, 4)$.

10.6 Devlin wishes to bound the quantifier $f$ by the set of $n$-sequences of finite sequences from $a$.

First problem: is it a set? No, even if $a$ has only two members: if $A$ is the class of $n$-sequences of finite sequences of members of $a$, the class $B$ of finite sequences of members of $a$ is a subclass of $\cup \cup \cup A$; and Model 5 is a supertransitive model of BS not containing the set $B I N$ of finite binary sequences, the reason being that $\overline{\overline{B I N \cap V_{n}}}=2^{n-3}$ for all $n \geqslant 3$; and hence in Model 5 , the class $A$ is not a set.

Second problem: would $B$ be a bounding class for the quantifier $\exists f$ ? No; it is the wrong type. The values of $f$ are not finite sequences but sets of finite sequences. 
However, the faulty proof of Lemma I.9.5 becomes true if we confine $a$ to being finite. First, a general lemma:

10.7 Proposition Let $G$ be a $\Delta_{0}$ class. Then

$$
\vdash_{\mathrm{DB}} \operatorname{Fn}(G) \& \operatorname{Dom}(G)=V \Longrightarrow \forall a(a \text { finite } \Longrightarrow G " a \in V)
$$

Proof : Let $f: n \longleftrightarrow a$. Consider the class $n \cap\left\{k \mid G\right.$ " $\left.\left\{\left.f(i)\right|_{i} i<k\right\} \notin V\right\}$. That is $\Pi_{1}$, and so if not empty, a minimal element exists, which, trivially, is $>0$, and hence equals $k+1$ for some $k$. Thus $G$ " $\left\{\left.f(i)\right|_{i} i<k\right\} \in V$; to that we must add $\{G(f(k))\}$.

10.8 REMARK Under the hypotheses of the Proposition $G$ " $a$ will be finite. 10.9 LEMMA (DB) If $a$ is finite, then for each $n$ there is a $u$ such that $\operatorname{Seq}(u, a, n)$. Hence for a finite, $\operatorname{Seq}(u, a, n) \Longleftrightarrow \forall u^{\prime} \neq u \neg \operatorname{Seq}\left(u^{\prime}, a, n\right)$.

Proof : by an induction on $n$; the induction step will require us to form $\left\{\left.x \cup y\right|_{x, y} x \in A \& y \in B\right\}$, where $A$ and $B$ are finite; but that is of the form $g$ " $(A \times B)$ where $g$ is rudimentary and provably total in DB, and thus satisfies the hypotheses of the Proposition. $A \times B$ will be finite by Proposition 2.17.

\section{Lemma 9.6 " $\mathrm{Fml}(x)$ is $\Delta_{1}^{B S}$ "}

This result is actually true, but the proof given is seriously flawed.

There is a slight error in the definition of $A(x)$; replace the third occurrence of ' $n$ ' by ' $m$ '.

At the bottom of the page, in the proof of Lemma 9.6, the claim "it is easily checked that

$$
B S \vdash \forall x \exists y[y=A(x)] . "
$$

is untrue; as is shown by Model 9, for appropriate infinite $x$.

However, this claim is needed only in the case that $x$ is a finite sequence, when the result is indeed provable:

10.10 LEMMA (ReS) If $x$ is a finite sequence, then $A(x)$ is a set.

Proof : Let $x$ be a finite set, and $k$ a finite ordinal. Then the set $B(k, x)$ of functions from $k$ to $x$ is a $\Delta_{0}$ subclass of $\mathcal{P}(x \times k)$, which as we have seen is, provably in $\mathrm{ReS}$, a set.

This principle, applied twice, yields our modified Lemma. $\quad \dashv(10.10)$

A final adjustment to the proof of 9.6: use the fact established above that Build is $\Delta_{1}^{\mathrm{BS}}$ twice: first to see that $\operatorname{Fml}(x)$ is $\Sigma_{1}$, since of the form $\exists f \operatorname{Build}(x, f)$; then to verify that the given alternative form is indeed $\Pi_{1}$, 
use Lemma 10.6 and Metatheorem 2.32 to show that the subformula $(\exists f: \in$ $v) \operatorname{Build}(x, f)$ is $\Pi_{1}^{\mathrm{BS}}$, since $v$ is a finite set.

\section{Lemma 9.7}

The above arguments, appropriately modified, will prove Lemma 9.7.

\section{The definition of Fr}

10.11 REMARK In the build-up to Lemma 9.8, one might comment that the definition of Fr involves a recursion on a finite tree. Perhaps there is some general principle that GJ suffices for such but that BS is too weak.

\section{Lemma 9.8: "Fr is $\Delta_{1}^{B S}$ "}

The Lemma is true but an appeal must be made again to Metatheorem 2.32: $F_{\wedge}$ occurs as a positive subformula, within the scope of quantifiers restricted by finite sets, themselves within the scope of two unrestricted existential quantifiers, and hence the formula $\operatorname{Fr}(\varphi, x)$ will indeed be $\Sigma_{1}^{B S}$.

There is also the point that the uniqueness of the $x$ for which $\operatorname{Fr}(\varphi, x)$ holds, should perhaps be proved, given the wide range of possible building sequences. The definition of Build as it stands says implicitly that one is considering a minimal subtree and that the recursion confined to that is all that is contributing to the final value.

\section{The definition of Sub}

In the build-up to Lemma 9.9, the phrase "the scope of this quantifier" is used but not defined.

\section{Lemma 9.9: "Sub is $\Delta_{1}^{B S "}$}

This again involves a recursion on a finite tree; otherwise how does one know that $\varphi^{\prime}$ exists?

The same problem, with the same cure, as for Lemma 9.8 , as $F_{\wedge}$ is used.

Fifth line from the bottom of page 39: for $F_{\in} \operatorname{read} F_{\exists}$.

\section{The definition of Sat}

Now Devlin gets down to defining Sat. But there is a real problem on page 41 for which our cure will not work: $w(u, \phi)$ may not be a set, as is shown by Model 9 . There is no chance of a cure as it involves $k$-sequences from an infinite set. Bad for a different reason; previously we had finite sequences of unbounded length from a finite set; here finite sequences of bounded length but from an infinite set. 
Lemma 9.10 "the LST formula $\operatorname{Sat}(u, \phi)$ is $\Delta_{1}^{B S}$ "

The statement is false, so this time there is no hope of saving the proof. In Model 6 , for no infinite set $x$ does there exist a $y$ with $\operatorname{Seq}(y, x, 4)$; for $u$ infinite, the set $a$ of names of members of $u$ will be infinite, and so the given $\Sigma_{1}$ formula for $\operatorname{Sat}(u, \varphi)$ will always be false; but then so is the $\Sigma_{1}$ version of $\operatorname{Sat}(u,\urcorner \varphi)$; but one of them ought to be true!

\section{Lemma 9.12}

The amended proofs of Lemmata 9.6 and 9.7 will now yield Lemma 9.12

\section{Errors in Chapter II}

\section{Amenability}

On page 45 , in section 10 , a set $M$ is defined to be amenable if it is transitive and satisfies 5 conditions: closed under pairing, sumsets, cart prod; contains omega; and closed under $\dot{\Delta}_{0}(M)$ separators, though Devlin writes " $\Sigma_{0} . "$

We have a curiosity here: the length of $\varphi$ is quantified in the language of discourse; it might well be that each $\varphi$ is in $M$; but if $M$ is non-standard it may think differently about what wffs are possible.

Given the ambiguity in the meaning of $\Delta_{0}$, I would suggest defining an amenable set as a transitive set containing $\omega$ and closed under the functions in the finite set of generators of the class $\mathcal{B}$.

On page 65 , in section 2 of Chapter 2 , he writes "by repeating the proof of I.9.10 for $\mathcal{L}$ in place of LST, we obtain a proof of the fact that the class Sat $(=\{(u, \varphi) \mid \operatorname{Sat}(u, \varphi)\})$ is uniformly $\Delta_{1}^{M}$ for amenable sets $M$. That is, there is a $\Sigma_{1}$ formula $\psi(x, y)$ of $\mathcal{L}$ and a $\Pi_{1}$ formula $\theta(x, y)$ of $\mathcal{L}$ such that for any amenable set $M$, if $u, \varphi \in M$ then

$$
\operatorname{Sat}(u, \varphi) \Longleftrightarrow \models_{M} \psi(\stackrel{\circ}{u}, \stackrel{\circ}{\varphi}) \Longleftrightarrow \models_{M} \theta(\stackrel{\circ}{u}, \stackrel{\circ}{\varphi}) .
$$

(The formulas $\psi$ and $\theta$ are just the $\mathcal{L}$ analogues of the LST formulas described in I.9.10.)"

With Model $\mathbf{M}_{6,5}$ in mind, we give a counterexample to the alleged uniformity for the specific formulation of Sat given by Devlin.

Let $u$ be an infinite transitive set containing only finitely many sets of cardinality 5. Let $M$ be the rud closure of $u \cup\{u\}$. Let $N$ be the union of the class of all transitive members of $M$ which have only finitely many sets of cardinality 5. So $u \in N$. Suppose we wish to evaluate the truth in $u$ of 
the sentence $\bigwedge x \bigvee y x \in y$ : readers will recognise that that is true in many $u$ and also false in many others. $M$ can correctly make that evaluation, (as we shall verify below); so the $\Pi_{1}$ form holds in $M$; therefore in $N$; therefore, if Devlin's assertion were correct, the $\Sigma_{1}$ form will hold in $N$. But it is false in $N$, because all atomic formulae such as $(x \in y)$ are sequences of length 5 , and therefore, $u$ being infinite, the set of atomic sentences of $\mathcal{L}_{u}$ is infinite and therefore not a member of $N$; and therefore not available to be the $f(0)$ of Devlin's formulation.

10.12 REMARK This argument suggests that no other pair of $\Pi_{1}$ and $\Sigma_{1}$ formulæ will work for amenable sets such as $N$, as information concerning the infinitely many atomic formulæ must be coded in some way into any truth-evaluation, which cannot therefore lie in $N$ if the said information can be recovered by some rudimentary function.

If one calls a set $M \mathcal{S}$-amenable if it is amenable and for each $x \in M$ $\mathcal{S}(x) \in M$, then Sat will indeed be uniformly $\Delta_{1}^{M}$ for $\mathcal{S}$-amenable sets $M$.

The discussion on page 66 seems to suggest that any statement which is $\Sigma_{1}^{\mathrm{KPI}}$ is $\Sigma_{1}$ over any $L_{\lambda}$ for limit $\lambda>\omega$, but such is of course not the case: consider the statement "there are are least three limit ordinals".

\section{Errors in Chapter VI}

Lemma VI.1.13 "Sat ${ }^{A}$ is $\Delta_{1}^{B S}$ "

The statement is false, being a generalisation of the false Lemma I.9.10.

Lemma VI.1.14 "truth for $\Delta_{0}$ wffs is uniformly $\Sigma_{1}$ for transitive rud-closed structures $\langle M, A\rangle$."

This ought to be correct, and it is of the greatest importance. We make some minor comments, but defer to a sequel, Rudimentary Recursion, a full discussion of the proof.

On page 242, in the proof of Lemma VI.1.14, the displayed formula in the middle of the page is incomplete as ' $t$ ' does not occur on the right-hand side. I suggest that the clause $f(\operatorname{Dom}(f)-1)=t$ should be added.

There is a delicate visual confusion of the meaning of brackets in the following subformula of that same displayed formula:

$$
\left(f(i)=\stackrel{\circ}{F}_{0} \dot{(}\left(f(j), f(k) \dot{)} \Longrightarrow g(i)=F_{0}(g(j), g(k))\right)\right.
$$

where the two parentheses that I have dotted are part of the syntax of the object language, not the language of discourse; but in Devlin's text no visual difference is made between them. Normally of course such confusion would 
cause no trouble, but in this particular context, greater exactitude might be desirable.

Lower on page 242, in line -7 , there is a typo: $t^{\varphi}$ should be $t_{\varphi}$.

Finally on page 243 , some correction will be needed as the troublemaker $F_{\wedge}$ recurs here and appeal is made to the false Lemma I.9.3.

The definition of $G_{\exists}$ oscillates between two and three variables.

On page 243 , line -5 , reference to 1.7 should perhaps be to 1.8 .

\section{Taking stock}

Much of the problem with Chapter I Section 9 has now been repaired, but the proposed definition of Sat is not possible in BS, and no other seems likely to succeed.

Hence I propose to show that my system DS is capable of defining Sat; and as it holds in all $J_{\nu}$ and $L_{\lambda}$ with $\lambda$ a limit ordinal $>\omega$ it might therefore be claimed to do the desired trick.

I shall also consider GJI, which also proves to define Sat.

On the other hand, the proof of VI.1.14 rests on a different idea, unrelated to the problems of defining Sat. The proof given by Devlin is tainted by its appeal to the false Lemma I.9.3, and therefore I propose in a sequel to rework the proof.

\section{The cure in DS}

We recall that DS is the theory $S_{0}+\Delta_{0}$ separation $+\Pi_{1}$ foundation $+\omega \in V+\mathcal{S}(x) \in V$.

$F_{\wedge}$ is $\Delta_{0}$ in the parameter $\mathcal{S}(\omega \times \omega)$, by the result, given as Corollary 8.16 that in DS the graph of each partial recursive function is a set. Further, corrected 9.3:

10.13 LEMMA $F_{\wedge}$ is $\Delta_{0, \mathcal{S}}^{\mathrm{DS}}$

corrected Lemma 9.4:

10.14 Lemma Build is $\Delta_{0, \mathcal{S}}^{\mathrm{DS}}$.

10.15 LEMMA (DS) $\forall a<\omega a \in V$.

Proof: Given $a$, each ${ }^{m} a \subseteq \mathcal{S}(a \times \omega)$. We may therefore use $\Delta_{0}$ separation to build a $u$ with $\operatorname{Seq}(a, u, n)$, since

$$
x \in \bigcup_{m<n}{ }^{m} a \Longleftrightarrow \underbrace{\operatorname{Fn}(x) \& \exists m:<n \operatorname{Dom}(x)=m \& \forall k:<m x(k) \in a}_{\Delta_{0}} .
$$

10.16 Proposition (DS) I.9.5 
Corrected I.9.10:

10.17 Lemma (DS) $w(u, \varphi) \in V$.

Proof : apply Lemma 10.7 and Proposition 10.16.

10.18 Proposition The LST formula Sat $(u, \phi)$ is $\Delta_{1}^{\mathrm{DS}}$

\section{The cure in GJI}

10.19 Lemma (GJ) $\forall n: \in \omega \forall a \exists u \operatorname{Seq}(u, a, n)$.

Proof : fix $a$; least failed $n$ is given by $\Pi_{1}$ foundation. then piece things together.

10.20 Proposition (GJ) I.9.5

10.21 Lemma (GJI) $w(u, \varphi) \in V$.

Proof : use the result and reasoning behind Theorem 2.93 .

10.22 REMARK The natural proof of Devlin I.9.6 would use $\Pi_{2}$ foundation to reduce the problem to showing that $\{\bigcup x \mid x \in a\}$ is a set, which is possible in GJ, but, by Model $\mathbf{M}_{7}$, not in DB.

10.23 Proposition The LST formula Sat $(u, \phi)$ is $\Delta_{1}^{\mathrm{GJ}}$.

Proof: with the existence of $w(u, \varphi)$ now established, we may follow Devlin's argument.

\section{Conclusion}

10.24 REMARK In the Introduction we spoke of three systems that might work in place of BS. One is our suggestion DS; the second is GJI, which apart from the restraint to $\Pi_{1}$ foundation, is the system RUD discussed in Stanley's review: but we see now that there is a third system, a subsystem of both those; namely the system DBI $+\forall a \forall k: \in \omega[a]^{k} \in V$, which proves Theorem 2.93, is a proper subsystem of GJI and a proper extension of DBI. Whether that third system will lend itself to an enhancement of its logic similar to the one, studied in $\S 8$, that was made possible by adding the axiom $\mathcal{S}(x) \in V$, must remain a question for another time.

10.25 REMARK I cannot claim to have checked through the whole book, but my remarks reassure me, if no-one else, that the errors are not catastrophic. A small change to the meaning of BS and all seems to be well.

\section{GANDY'S INEXACT REMARKS}

Gandy in $[\mathrm{G}]$ says of his four weak set theories PZ, BST', BRT and PZF, that were one to drop the requirement of $\Delta_{0}$ the four would stretch from 
Zermelo to Zermelo-Fraenkel, and continues "presumably these are also all distinct". His first remark is prima facie false as he makes no mention of the power set axiom (nor of the axiom of foundation) and the power set axiom is certainly independent of the others as (working say in ZFC) HC satisfies all other axioms of ZF.

We insert BS in the sequence and comment on the effect on the five of dropping the restriction to $\Delta_{0}$, of adding the power set axiom, and of doing both.

\section{The full systems without power set}

The first system will have axioms of extensionality, pairset, sumset and infinity, and the full separation scheme. The second system will add Cartesian product to that.

The model $\mathbf{M}_{2}$ satisfies full separation but not Cartesian product.

Corresponding to GJ, we have the full RUD replacement scheme:

(full $R R$ )

$$
\forall x \exists w \forall v: \in x \exists t: \in w \forall u(u \in w \Longleftrightarrow . u \in x \& \phi[u, v]) .
$$

for $\phi$ any formula.

The model $\mathbf{M}_{7}$ satisfies full separation and Cartesian product, but witnesses a failure of (restricted) rudimentary replacement.

Corresponding to fReR we have the full flat replacement axiom: namely, for any $\phi$, (full flat repl.)

$\forall x: \in u \exists ! y(\phi(x, y) \& y \subseteq z) \Longrightarrow \exists u \forall y[y \in v \Longleftrightarrow \exists x: \in u(\phi(x, y) \& y \subseteq z)]$

But full flat replacement is derivable from full rudimentary replacement, using the self-strengthening of full RR corresponding to that noted in Proposition 2.88 for $\mathrm{RR}$, by remarking that the set promised by an instance of full flat replacement is of the form

$$
\{Z \cap\{y \mid \exists Y \Phi(X, Y) \& y \in Y\} \mid X \in U\} .
$$

So in fact the distinction between the two systems will collapse already at $\Sigma_{1}$.

As for full flat collection, full replacement and full collection, Gandy's choice $\mathbf{G}_{3}=V_{\omega+\omega}$ gives a model of full flat collection in which replacement fails - but since gfReR is a subsystem of $\mathbf{Z}$, we may also find a model for it in which HF does not exist - and Zarach's model, [Z] Theorem 6.4, gives a model of full replacement in which collection, possibly even flat collection, fails. 


\section{Gandy's systems with added power set}

$\mathrm{PZ}+\mathrm{P}$ is the system $\mathrm{M}_{0}$, in which Cartesian product is provable, as are Rudimentary Replacement, and flat $\Delta_{0}$ Replacement and Collection. $\mathrm{PZF}+\mathrm{P}$ is strictly stronger, as it builds $\omega+\omega$.

\subsection{Problem Is KPI $+\mathbf{P}$ the same as $\mathrm{ReR}+\mathbf{P}$ ?}

\section{The full systems with foundation and power set added}

We have just $Z$ in the first case; and the first four cases now coincide, for full flat replacement is provable in Z, just as fReR is provable in $M_{0}$ using power set plus $\Delta_{0}$ separation. The fifth is ZF.

\section{A MODEL OF Z PLUS FULL Foundation IN WHICH TCo FAILS}

Boffa [B1] [B2] has constructed two other models of $Z+\neg T$ Co; ours appears to be a third.

12.0 Definition $\iota^{0}(x)=_{\mathrm{df}} x ; \iota^{n+1}(x)==_{\mathrm{df}}\left\{\iota^{n}(x)\right\}$.

12.1 Definition $\varrho$ is the set-theoretical rank of $x$.

12.2 Definition $V_{n}==_{\mathrm{df}}\{x \mid \varrho(x)<n\} ; b_{n}==_{\mathrm{df}} \iota^{n}\left(V_{n}\right)$.

12.3 Definition For each $n \in \omega$, set $c_{n}=_{\text {df }}\left\{\bigcup^{n} b_{m} \mid n \leqslant m<\omega\right\}$.

12.4 EXAmple $c_{0}=\left\{V_{0},\left\{V_{1}\right\},\left\{\left\{V_{2}\right\}\right\}, \ldots\right\} ; c_{1}=\left\{V_{1},\left\{V_{2}\right\},\left\{\left\{V_{3}\right\}\right\}, \ldots\right\} ;$ $c_{2}=\left\{V_{2},\left\{V_{3}\right\},\left\{\left\{V_{4}\right\}\right\}, \ldots\right\}$

12.5 Proposition $\bigcup c_{n}=V_{n} \cup c_{n+1}$.

12.6 Definition $K_{0}=_{\mathrm{df}} \omega \cup\left\{c_{0}\right\} ; K_{n+1}=_{\mathrm{df}} \mathcal{P}\left(K_{n}\right) \cup K_{n} \cup c_{n} ; K==_{\mathrm{df}}$ $\bigcup_{n \in \omega} K_{n}$

12.7 THEOREM $K$ is a supertransitive model of Zermelo set theory Z in which some set is a member of no transitive set.

12.8 LEMma $K_{n} \subseteq K_{n+1}$, and $K_{n} \in K_{n+1} \subseteq K$, so that each $K_{n} \in K$.

12.9 Corollary $K$ models Pairing.

12.10 LEMMA $V_{n} \subseteq K_{n}$.

Proof : induction on $n$. $V_{0}=\varnothing$; if $V_{n} \subseteq K_{n}, V_{n+1}=\mathcal{P}\left(V_{n}\right) \subseteq \mathcal{P}\left(K_{n}\right) \subseteq$ $K_{n+1}$.

12.11 Corollary $K$ includes all of $V_{\omega}=\mathbf{H F}$; in particular $K$ contains all finite ordinals. Moreover $\omega \in K_{1} \subseteq K$.

12.12 LEMMA $K$ is transitive: 
A. R. D. MATHIAS

Proof : Let $x \in y \in K_{0}$. Then either $y \in \omega$ when $x \in K$ or $y=c_{0}$ when $x \in \mathbf{H F} \subseteq K$.

Let $x \in y \in K_{n+1}$. then either $y \subseteq K_{n}$, when $x \in K_{n}$, or $y \in K_{n}$, when inductively we have already shown that $x \in K$; or $y \in c_{n} \subseteq \mathbf{H F}$, when $x \in \mathbf{H F} \subseteq K$.

12.13 Corollary $K$ models Extensionality, Null Set, Infinity and (full) Foundation.

12.14 LEMMA $K$ is supertransitive,

Proof : $x \subseteq y \in K_{n} \Longrightarrow x \in K_{n+1} \in K$.

12.15 Corollary $K$ is a model of full Separation.

12.16 Lemma Each $\bigcup K_{n}$ is a subset of $K_{n+1}$ and thus is in $K$ by supertransitivity.

Proof: $\bigcup K_{0}=\omega \cup c_{0} \subseteq K_{1} \in K$. If $\bigcup K_{n} \subseteq K_{n+1}, \bigcup K_{n+1}=K_{n} \cup \bigcup K_{n} \cup$ $V_{n} \cup c_{n+1} \subseteq K_{n+2}$.

12.17 Corollary $K$ models Union.

Proof: if $y \in K_{n}$, then $y \subseteq \bigcup K_{n} \subseteq K_{n+1}$, so $\bigcup y \subseteq \bigcup K_{n+1} \in K$, so $\bigcup y$ is in $K$.

12.18 Lemma $K$ models Power set.

Proof : If $x \in K_{n}, x \subseteq K_{n+1}$ so $\mathcal{P}(x) \subseteq \mathcal{P}\left(K_{n+1}\right) \subseteq K_{n+2}$.

Thus we have shown that $K$ models $\mathrm{Z}$.

12.19 Proposition $\forall n \forall m\left[m \geqslant n+3 \Longrightarrow V_{m} \notin K_{n}\right]$.

Proof: $V_{0}=0 ; V_{1}=1, V_{2}=2$ but for $m \geqslant 3, V_{m}$ is not an ordinal and is therefore not in $\omega$, nor is it, a finite set, equal to $c_{0}$, an infinite set. Hence $V_{3} \notin K_{0}$.

Suppose that $V_{m} \notin K_{n}$, for any $m \geqslant n+3$. If $V_{m+1} \in K_{n+1}$, then either $V_{m+1} \subseteq K_{n}$, so that $V_{m} \in K_{n}$, contradicting the inductive hypothesis; or $V_{m+1} \in K_{n}$, again contrary to the inductive hypothesis; or $V_{m+1} \in c_{n}=$ $\left\{V_{n},\left\{V_{n+1}\right\},\left\{\left\{V_{n+2}\right\}\right\} \ldots\right\}$, again impossible by inspection. $\quad \dashv(12.19)$

12.20 Proposition TCo fails in $K$.

Proof : $c_{0} \in K$. Suppose that $c_{0} \in u \in K$ with $u$ transitive. Then $\mathbf{H F} \subseteq u$, so $\mathbf{H F} \in K$, and hence $H F \in K_{n}$ say, so that $\mathbf{H F} \subseteq K_{n+1}$. But $K_{n+1}$ contains at most $n+4$ of the sets $V_{m}$.

Other constructions of models of Zermelo are given in Slim Models. The constructions there furnish an entertaining independence argument for the axiom of pairing, which we shall give in the next section. 


\section{AxPair AND AxSing}

Let $\mathbf{Z}$ be Zermelo set theory, including the axioms of infinity and foundation. Let TCo be the assertion that every set is a member of a transitive set. Let TIn be the assertion that every set is a subset of a transitive set. Let AxSing be the assertion that for each set $x,\{x\}$ is a set. Let AxPair be the assertion that for all sets $x$ and $y,\{x, y\}$ is a set.

13.0 REMARK TCo trivially (in the strict sense) implies TIn; TIn + AxSing implies TCo. AxSing is usually derived from AxPair, either by taking $x=y$ or if AxPair is confined to the strict case, by using separation. Indeed AxSing is provable using separation and power set, since each set $x$ is a member of its power set, should the latter exist.

We shall exhibit a model of almost all of Zermelo, in which AxSing is true but AxPair is false, and a model of a substantial amount of set theory in which TIn holds but AxSing and TCo fail.

It is amusing to note that in the system of Bourbaki, the pairing axiom has been proved to be redundant. see Sonner [S]. That it is not redundant in Z was first shown by Boffa [B3].

\section{Failure of AxPair}

Let $\mathrm{T}$ be the theory $\mathrm{Z}+\mathrm{TC}$ + + WO,- - WO being the statement "every set has a well-ordering" - and let $\mathrm{T}^{-}$be the theory $\mathrm{T}$ with the axiom of pairing replaced by its negation: $\exists x \exists y\{x, y\} \notin V$, and with the addition of AxSing.

13.1 REMARK The scheme of foundation for all classes is provable in $\mathrm{T}^{-}$.

We show that if $\operatorname{Consis}(\mathrm{Z})$ then $\operatorname{Consis}\left(\mathrm{T}^{-}\right)$.

It follows from the last part of Theorem 5 of The Strength of Mac Lane Set Theory [M2], proved in Section 5 of that paper, that if $\mathbf{Z}$ is consistent, so is $\mathrm{Z}+\mathrm{KP}+\mathrm{WO}$.

A set or class $\mathbf{M}$ is said to be supertransitive if it is transitive and, further, $x \subseteq y \in \mathbf{M} \Longrightarrow x \in \mathbf{M}$.

As in the proof of Theorem 4.8 of Slim Models of Zermelo Set Theory [M1] one can, working in the theory $\mathrm{Z}+\mathrm{KP}+\mathrm{WO}$, build two supertransitive models $\mathbf{M}$ and $\mathbf{N}$ of $\mathbf{Z}+$ TCo + WO, with neither a subset of the other: e.g. take $\mathbf{M}$ to contain $Z(0)$ but not $Z(\omega)$ and $\mathbf{N}$ to contain $Z(\omega)$ but not $Z(0)$, in the notation of that paper. Further one can choose $\mathbf{M}$ and $\mathbf{N}$ to contain all ordinals, all sequences of ordinals and all sets of sequences of ordinals.

THEOREM Let $\mathbf{M}$ and $\mathbf{N}$ be supertransitive models of $\mathbf{T}$, neither included in the other; then $\mathbf{M} \cup \mathbf{N}$ is a model of $\mathrm{T}^{-}$. 
Proof : Remark first that $\mathbf{M} \cup \mathbf{N}$ is supertransitive, and hence absolute for most of the set-theoretical concepts used in the axioms; therefore it will be a model of Extensionality, Sum Set, Power Set, full Separation, Foundation, TCo (whence also Foundation for all classes), and WO.

[For power set, use supertransitivity; otherwise there would be a risk of $\mathbf{N}$ containing subsets of some element of $\mathbf{M}$ which were not in $\mathbf{M}$. Supertransitivity also gives the truth of full separation in $\mathbf{P}$. I think that for the other axioms the transitivity of $\mathbf{P}$ is enough.]

Pairing fails, for if $a \in \mathbf{M} \backslash \mathbf{N}$ and $b \in \mathbf{N} \backslash \mathbf{M}$, then $\{a, b\} \notin \mathbf{M} \cup \mathbf{N}$. But AxSing holds.

METACOROLLARY If $\mathbf{Z}$ is consistent so is $\mathrm{T}^{\prime}$.

13.2 REMARK In $\mathbf{P}$, the following curious axiom holds: given three sets, $a, b, c$ at least one of the three classes $\{a, b\},\{b, c\}\{c, a\}$ is a set. That suggests numerous variations on the construction, well-supported by the plethora of incomparable models constructed in [M1].

13.3 REMARK Boffa in [B3] shows of every member $a$ of $\mathbf{H F}$ that it is provable in $\mathbf{Z}$ that for any $x$, the pair $\{a, x\}$ exists: for example both the empty set and $x$ are in $\mathcal{P}(x)$, and therefore the pair $\{\varnothing, x\}$ can be recovered using Separation. Thus the smallest possible set which might not form a pair with something is of rank at least $\omega$, and Boffa shows that the set $\{\varnothing,\{\varnothing\},\{\{\varnothing\}\} \ldots\}$ of Zermelo integers indeed has that property.

\section{Failure of AxSing}

Consider, working in some suitable theory such as ZF, the class $\mathbf{C}$ of all sets $x$ such that $\operatorname{tcl}(x)$ contains at most one strict pair, that is, a set of the form $\{b, c\}$ with $b \neq c$. $\mathbf{C}$ is supertransitive, and models "much" of Z: namely Extensionality, full separation, sum set, and infinity; and it contains all the ordinals, of which $2=\{0,1\}$ is the only strict pair. AxSing fails since $\{5,6\}$ is a member of $\mathbf{C}$ but $\{\{5,6\}\}$ is not. AxPair fails since $\{7,8\}$ is in C but $\{\{5,6\},\{7,8\}\}$ is not.

Moreover TIn holds in $\mathbf{C}$, since the transitive closure of an element of $\mathbf{C}$ is itself an element of $\mathbf{C}$; but TCo is false, since for example $\{5,6\}$ cannot be a member of any transitive element of $\mathbf{C}$.

14. A QUESTION OF MACALOON ON RUDIMENTARY CLOSURES.

Let $\mathbb{T}$ be the rudimentary function of Definition 2.53 .

14.0 LEMMA Let $\left\langle u_{n} \mid n \in \omega\right\rangle$ be any sequence of transitive sets. Define

$$
K_{0}=u_{0} ; \quad K_{n+1}=\mathbb{T}^{5}\left(K_{n}\right) \cup u_{n} \quad K_{\omega}=\bigcup_{n<\omega} K_{n} .
$$


Then $K_{\omega}$ is rud closed.

Proof: We show that $K_{\omega}$ is closed under each of the functions $R_{0}$ to $R_{8}$. By the properties of $\mathbb{T}$ established in $\S 2$ following Definition 2.73, $x, y$ in $u$ implies $R_{i}(x) \in \mathbb{T}(u)$ for $\mathrm{i}=2,3,5$; and $x, y$, in $u$ implies $R_{i}(x, y) \in \mathbb{T}(u)$ for $\mathrm{i}=0,1 ; x$ in $u$ implies $R_{i}(x) \in \mathbb{T}^{5}(u)$ for $\mathrm{i}=6,7 ; x, y$ in $u$ implies $R_{4}(x, y) \in \mathbb{T}^{3}(u)$; and $x, y$ in $u$ implies $R_{8}(x, y) \in \mathbb{T}^{2}(u)$. $K_{n+1}$.

As $u \subset \mathbb{T}(u) \subset \mathbb{T}^{2}(u) \ldots$, it follows that for each $n, K_{n} \subseteq \mathbb{T}^{5}\left(K_{n}\right) \subseteq$

14.1 Definition $\iota(x)=_{\text {df }}\{x\}$

14.2 Lemma If $x \notin u$ then $\iota(x) \notin \mathbb{T}(u)$; and hence $\iota^{4}(x) \notin \mathbb{T}^{4}(u)$.

Proof : every member of $\mathbb{T}(u)$ is a subset of $u$.

14.3 Proposition Suppose that $u$ is a transitive set closed under pairing. Then whenever $w$ is a transitive set of which $u$ is not a subset, $u$ is not a member of the rud closure of $u \cup w$.

Proof : $u$ must be of limit rank $\lambda$ say.

Suppose first that $u$ is countable, so that $\lambda$ is of cofinality $\omega$. Let $\lambda_{n} \nearrow_{n} \lambda$. We fix an enumeration of $x$ and use it to make the following choices.

Pick $x_{0} \in u \backslash w$. Let $u_{0}=u \cap V_{\max \left\{\lambda_{0}, \varrho\left(x_{0}\right)+1\right\}}$.

Pick $x_{1} \in u \backslash u_{0}$, with $\iota^{4} x_{0} \in x_{1}$. Let $u_{1}=u \cap V_{\max \left\{\lambda_{1}, \varrho\left(x_{1}\right)+1\right\}}$.

Pick $x_{n+1} \in u \backslash u_{n}$, with $\iota^{4} x_{n} \in x_{n+1}$. Let $u_{n+1}=u \cap V_{\max \left\{\lambda_{n+1}, \varrho\left(x_{n+1}\right)+1\right\}}$.

Finally let $K_{0}=w ; K_{n+1}=\mathbb{T}^{5}\left(K_{n}\right) \cup u_{n} ; K_{\omega}=\bigcup_{n} K_{n}$.

Then every $K_{n}$ is transitive and by the Lemma, $K_{\omega}$ is rud closed, and includes $w \cup\{w\} \cup u$. If $x_{n+1} \in K_{n+1}$, it cannot, by construction, be a member of $u_{n}$ and so must be a subset of $\mathbb{T}^{4}\left(K_{n}\right)$, so $\iota^{4}\left(x_{n}\right) \in \mathbb{T}^{4}\left(K_{n}\right)$, which by Lemma 14.2 implies $x_{n} \in K_{n}$. But $x_{0} \notin K_{0}$; so by induction no $x_{n} \in K_{n}$. Hence no superset of $\left\{x_{n} \mid n \in \omega\right\}$ can be a member of $K_{\omega}$. In particular, $u$ cannot be.

The Proposition is now proved for the case that $u$ is countable. In the general case, go to a generic extension of the universe in which $u$ is countable; the hypotheses will still hold; hence in the generic extension, $u$ is not in the rud closure of $u \cup w \cup\{w\}$; but that latter statement is absolute and therefore true in the ground model.

14.4 Corollary Let $u$ be transitive and closed under pairing; then $u$ is not in the rud closure of $O N \cup u$.

A particular case answers a question posed by McAloon in the 1970's:

14.5 COROLlary For any $\alpha>0, J_{\alpha} \notin \operatorname{rud} \operatorname{cl}\left(J_{\alpha} \cup\{\omega \alpha\}\right)$

I thank Lee Stanley for telling me of McAloon's question. 
A. R. D. MATHIAS

14.6 REMARK So far as the definition of $K_{\omega}$ goes, other functions $T$ could be used instead of $\mathbb{T}$, provided they had the property that the members of $T(u)$ are subsets of $u$ : for example, if we instead use $u \mapsto \mathcal{P}(u), K_{\omega}$ will be a model of Zermelo set theory, probably including the axiom of infinity, though possibly not in the form $\omega \in V$ : we adopt this strategy in the following variant.

14.7 Proposition Suppose that $\left(x_{n}\right)_{n}$ and $\left(u_{n}\right)_{n}$ are two sequences of sets such that for each $n<\omega$ :

(14.7.0) $x_{n} \in u_{n}$;

(14.7.1) $u_{n} \subseteq u_{n+1}$;

(14.7.2) $u_{n}$ is transitive;

(14.7.3) $x_{n} \in \operatorname{tcl}\left(x_{n+1}\right)$;

(14.7.4) $x_{n+1} \notin u_{n}$.

Then $\bar{u}=\mathrm{df} \bigcup_{n} u_{n}$ is transitive and if $w$ is a transitive set with $x_{0} \notin w$, the set $\bar{x}={ }_{\mathrm{df}}\left\{x_{n} \mid n \in \omega\right\}$ is not a member of the rud closure of $\bar{u} \cup w \cup\{w\}$. If in addition $\omega \subseteq w$, then there is a supertransitive model of Zermelo set theory of which $\bar{u} \cup w \cup\{w\}$ is a subset but $\bar{x}$ and $\bar{u}$ are not members.

Proof : Let $K$ be the model formed as follows:

$$
K_{0}=w ; \quad K_{n+1}=\mathcal{P}\left(K_{n}\right) \cup u_{n} ; \quad K=\bigcup_{n} K_{n} .
$$

Then each $K_{n}$ is transitive.

14.8 LEMMA Each $K_{n}$ is a member of $K_{n+1}$.

14.9 LEMMA $K_{0} \subseteq K_{1}$; if $K_{n} \subseteq K_{n+1}$ then $K_{n+1} \subseteq K_{n+2}$.

Proof: As $K_{0}$ is transitive, its members are also subsets of it and therefore members of $K_{1}$. Under the hypotheses of the second statement, $\mathcal{P}\left(K_{n}\right) \subseteq$ $\mathcal{P}\left(K_{n+1}\right) \subseteq K_{n+2}$ and $u_{n} \subseteq u_{n+1} \subseteq K_{n+2}$.

14.10 LEMMA $\bigcup K_{0} \subseteq K_{0} ; \bigcup K_{n+1}=K_{n} \cup \bigcup u_{n}$.

14.11 Lemma If $x \in K$, then for some $\ell, x \subseteq K_{\ell}$.

14.12 LEMMA $K$ is transitive.

Proof: If $y \in x \in K$ then for some $\ell, y \in x \subseteq K_{\ell}$, so $y \in K_{\ell} \subseteq K$.

14.13 LEMMA $K$ is supertransitive.

Proof :If $y \subseteq x \in K$ then for some $\ell, y \subseteq x \subseteq K_{\ell}$, so $y \in \mathcal{P}\left(K_{\ell}\right) \subseteq K_{\ell+1} \subseteq$ $K$.

14.14 COROLlary $K$ models the full separation scheme. 
14.15 LEMMA $x \in K \Longrightarrow \mathcal{P}(x) \in K$.

Proof : by Lemma 14.12, $x$ is a subset of some $K_{\ell}$; by the proof of Lemma 14.14, any subset of $x$ is in $K_{\ell+1}$, and so $\mathcal{P}(x)$ is a subset of $K_{\ell+1}$ and therefore a member of $K_{\ell+2}$.

14.16 LEMMA Each $\bigcup K_{n}$ is in $K$.

Proof : by supertransitivity, as each $K_{n} \in K$.

14.17 LEMMA $x \in K \Longrightarrow \bigcup x \in K$.

Proof: If $x \subseteq K_{\ell}$, then $\bigcup x \subseteq \bigcup K_{\ell}$, which is in $K$; as $K$ is supertransitive, $\bigcup x \in K$.

$\dashv(14.17)$

14.18 LEMMA For no $n$ is $x_{n}$ a member of $K_{n}$; hence $\bar{x}$ is a subset of no $K_{n}$; hence neither it not $\bar{u}$ can be a member of $K$.

Proof: $x_{0} \notin K_{0}$ by hypothesis. Suppose that $x_{n+1} \in K_{n+1}$, then either $x_{n+1} \subseteq K_{n}$, giving $x_{n} \in K_{n}$, (since $K_{n}$ is transitive) or else $x_{n+1} \in u_{n}$, contrary to hypothesis.

So $x_{n} \notin K_{n} \Longrightarrow x_{n+1} \notin K_{n+1}$; by induction, for no $n$ is $x_{n}$ a member of $K_{n}$; as $x_{n} \in \bar{x}, \bar{x} \nsubseteq K_{n}$. Lemma 14.12 now implies that $\bar{x}$ is not a member of $K$; as it is a subset of $\bar{u}$ and $K$ is supertransitive, $\bar{u}$ cannot be a member of $K$.

14.19 LEMMA $\bar{u} \cup w \cup\{w\} \subseteq K$.

14.20 Lemma If $x \in K_{m}$ and $y \in K_{n}$ then for $\ell=\max (m, n),\{x, y\} \subseteq K_{\ell}$ and so is in $K$.

14.21 Proposition $\omega \in K \Longleftrightarrow \omega \subseteq w$.

14.22 Proposition $K$ is a model of all axioms of Zermelo set theory except possibly the axiom of infinity.

14.23 Corollary $K$ is rud closed.

14.24 REMARK If we take $u=\mathbf{H F}$ and $w=\omega, K_{\omega}$ will be a set model of Zermelo of which $\mathbf{H F}$ is not a member. Thus our argument generalises constructions to be found in the texts of Moschovakis and Enderton.

A third possibility is in the proof of the next remark.

14.25 Proposition Let $u$ be transitive and be the strictly increasing union of a sequence $u_{n}$ of transitive sets with $u_{0}$ not an ordinal and $u_{n} \in u_{n+1}$. Let $\zeta=O N \cap u$. Then the rud closure of $u \cup\{\zeta\}$ is a proper subset of the rud closure of $u \cup\{u\}$.

Proof : define $K_{0}=\zeta ; K_{n+1}=\operatorname{Def}\left(K_{n}\right) \cup u_{n} ; K=\bigcup_{n} K_{n}$.

$K$ is rud closed and includes $u \cup\{\zeta\}$; but one may show that each $u_{n} \notin K_{n}$; hence $u \notin K$. 


\section{AN APPLICATION TO GANDY NUMERALS}

The method of Section 14 casts some light on the proposal made by Gandy in $[\mathrm{G}]$ for discarding the von Neumann ordinals as numerals for the purpose of developing formal syntax. Their problem is that the rank of $n$ is $n$. His method makes use of ideas of Smullyan [Sm].

\section{First step: $\hat{\omega}$}

15.0 Definition We assign to each $n \in \omega$ a hereditarily finite set $\hat{n}$ and a level $\lambda(n) \in \omega$.

$\hat{0}=0 ; \hat{1}=\{0\} ; \lambda(0)=\lambda(1)=0$.

For $n>0$ let $n-1=\Sigma_{\ell<k} a_{\ell} 2^{\ell}$, where $a_{\ell} \in\{1,2\}$. Then put

$$
\hat{n}=\left\{\left\{\hat{\ell} \mid \ell<k \& a_{\ell}=2\right\},\{\hat{\ell} \mid \ell<k\}\right\} ; \lambda(n)=k .
$$

15.1 Example $\hat{2}=\{0,\{0\}\} ; \hat{3}=\{\{0\}\} ; \lambda(2)=\lambda(3)=1$

$$
\hat{4}=\{0,\{0,\{0\}\}\} ; \hat{5}=\{\{0\},\{0,\{0\}\}\} ; \hat{6}=\{\{\{0\}\},\{0,\{0\}\}\} ; \hat{7}=
$$
$\{\{0,\{0\}\}\} ; \lambda(4)=\lambda(5)=\lambda(6)=\lambda(7)=2$.

Set $\hat{\omega}=\{\hat{n} \mid n \in \omega\}$.

To get $\lambda$ we need the graph of exponentiation.

\section{Second step: $\bar{\omega}$}

Then set $\bar{n}={ }_{\mathrm{df}}\{\hat{m} \mid m<n\}$ and $\bar{\omega}={ }_{\mathrm{df}}\{\bar{n} \mid n \in \omega\}$.

It is the members of $\bar{\omega}$ that Gandy proposes, and which we shall call Gandy numerals. He proves that

the predicate $x \in \bar{\omega}$ is $\Delta_{0}$; addition and multiplication of Gandy numerals are rudimentary; concatenation of sequences of Gandy numerals is rudimentary; but exponentiation of Gandy numerals is not rudimentary.

His reason for not remaining with $\hat{\omega}$ is that he was unable to prove that $x \in \hat{\omega}$ is $\Delta_{0}$, and he speculated that $x \in \hat{\omega}$ is in fact not.

15.2 Proposition Neither $\hat{\omega}$ nor $\bar{\omega}$ is in rud $\operatorname{cl}(\{\omega\})$.

Proof : we apply Proposition 14.7. $\hat{0}=0, \hat{1}=1, \hat{2}=2$ but $\hat{3}=\{1\}$ which is not an ordinal. Therefore let $x_{0}=\hat{3}$, and $u_{0}=\operatorname{tcl}\left(\left\{x_{0}\right\}\right)$. Let $x_{n+1}$ be $\hat{k}$ for $k$ the least such that $\hat{k} \notin u_{n}$ and $x_{n} \in \operatorname{tcl} \hat{k}$; take $u_{n+1}=u_{n} \cup \operatorname{tcl}\left(\left\{x_{n+1}\right\}\right)$. The resulting supertransitive model $K$ is rud closed and does not contain $\bar{x}$; therefore it does not contain $\hat{\omega}$, of which $\bar{x}$ is a subset. But it does include the rudimentary closure of $\{\omega\}$.

Since $\bigcup \bar{\omega}=\hat{\omega}, \bar{\omega}$, too, cannot be in $K$. 
15.3 REMARK We can define a version, $\widehat{\mathrm{ACK}}$, of the Ackermann relation by $\hat{m} \widehat{\mathrm{ACK}} \hat{n}={ }_{\mathrm{df}} \hat{m} \in \bigcap \hat{n}$.

By the Proposition, $\hat{\omega}$ is not provably a set in GJ. But in GJ, we can show that if $\hat{\omega}$ is a set, then so is the relation $\widehat{A C K}$, and therefore the set of all finite subsets of $\hat{\omega}$ will be obtainable as $\{\widehat{\operatorname{ACK}} "\{x\} \mid x \in \hat{\omega}\}$.

\section{Acknowledgments}

This paper owes its existence to my participation in the Set Theory Year, 2003-4, at the Centre de Recerca Matemàtica at Bellaterra outside Barcelona: I express my gratitude to Joan Bagaria and the organisers of the Set Theory Year for their invitation; to my fellow-members of the research group ERMIT at the Université de la Réunion, who enabled me to maximise my stay at the CRM; to the Director and staff of the CRM for the excellent working conditions that they continue to provide; to the members of the set theory seminars in Réunion and at the CRM who patiently heard successive versions of this material; and to Thomas Forster, Kai Hauser, Ronald Jensen, Robert Lubarsky, Colin McLarty, Thoralf Räsch and Lee Stanley for their stimulating encouragement and helpful observations.

\section{REFERENCES}

[B1] Boffa, Maurice, Axiome et schéma de fondement dans le système de Zermelo, Bull Acad Polon Sci Sér Math Astron Phys 171969 113-5. MR 40 \# 38.

[B2] Boffa, Maurice Axiom and scheme of foundation, Bull Soc Math Belg 22 (1970) 242-247; MR 45 \#6618.

[B3] Boffa, Maurice, L'axiome de la paire dans le système de Zermelo, Arch. Math. Logik Grundlagenforschung 15 (1972) 97-98. MR 47 \#6486

[Del] Ch. Delhommé, Automaticité des ordinaux et des graphes homogènes (Automaticity of ordinals and of homogeneous graphs) C. R. Acad. Sci. Paris, Ser. I 339 pp 5-10 (2004). (French with abridged English version)

[Dev] K. Devlin, Constructibility, Perspectives in Mathematical Logic, Springer-Verlag, Berlin, 1984.

[Do] A. J. Dodd, The Core Model, London Mathematical Society Lecture Note Series, 61, Cambridge University Press, 1982. MR 84a:03062.

[DoMT] J. E. Doner, A. Mostowski and A. Tarski, The elementary theory of well-ordering: a metamathematical study, in Logic Colloquium 'rr7, ed 
A. R. D. MATHIAS

A. Macintyre, L. Pacholski, J. Paris, North-Holland Publishing Company, 1978, 1-54.

[G] R. O. Gandy, Set-theoretic functions for elementary syntax, in Proceedings of Symposia in Pure Mathematics, 13, Part II, ed. T.Jech, American Mathematical Society, 1974, 103-126.

[J1] R. B. Jensen, Stufen der konstruktiblen Hierarchie. Habilitationsschrift, Bonn, 1967 (?)

[J2] R. B. Jensen, The fine structure of the constructible hierarchy, with a section by Jack Silver, Annals of Mathematical Logic, 4 (1972) 229-308; erratum ibid 4 (1972) 443.

[M1] A. R. D. Mathias, Slim models of Zermelo Set Theory, Journal of Symbolic Logic 66 (2001) 487-496.

[M2] A. R. D. Mathias, The Strength of Mac Lane Set Theory, Annals of Pure and Applied Logic, 110 (2001) 107-234.

[M3] A. R. D. Mathias, A note on the schemes of replacement and collection, to appear in the Archive for Mathematical Logic.

[M4] A. R. D. Mathias, Rudimentary recursion, in preparation.

[M5] A. R. D. Mathias, Rudimentary forcing, in preparation.

[Sm] R. M. Smullyan, Theory of formal systems, Ann. of Math. Studies, no 3, Princeton University Press, Princeton N.J., 1940 . MR 2, 66.

[So] Sonner, Johann, On sets with two elements, Arch. Math (Basel) 20 (1969) 225-7. MR 40 \#36.

[St] Stanley, Lee, review of [De], Journal of Symbolic Logic 53(1982 ?) 8648.

[Z] Zarach, Andrzej M., Replacement $\nrightarrow$ Collection, in Gödel '96, ed. Petr Hájek, (Springer Lecture Notes in Logic, Volume 6). 